University of Louisville

ThinkIR: The University of Louisville's Institutional Repository

Electronic Theses and Dissertations

$8-2010$

\title{
Enhanced cryptographic approaches for SCADA network security.
}

Waleed H. ElSaid

University of Louisville

Follow this and additional works at: https://ir.library.louisville.edu/etd

\section{Recommended Citation}

EISaid, Waleed H., "Enhanced cryptographic approaches for SCADA network security." (2010). Electronic Theses and Dissertations. Paper 403.

https://doi.org/10.18297/etd/403

This Doctoral Dissertation is brought to you for free and open access by ThinkIR: The University of Louisville's Institutional Repository. It has been accepted for inclusion in Electronic Theses and Dissertations by an authorized administrator of ThinkIR: The University of Louisville's Institutional Repository. This title appears here courtesy of the author, who has retained all other copyrights. For more information, please contact thinkir@louisville.edu. 


\title{
ENHANCED CRYPTOGRAPHIC APPROACHES FOR SCADA NETWORK SECURITY
}

\author{
By \\ Waleed H. ElSaid \\ BS., Ain Shams University, Egypt, 1993 \\ MS., Ain Shams University, Egypt, 2000

\begin{abstract}
A Dissertation
Submitted to the JB. Speed School of Engineering

of the University of Louisville

in Partial Fulfillment of the requirements

for the Degree of

Doctor of Philosophy
\end{abstract} \\ Department of Computer Engineering and Computer Science \\ JB. Speed School of Engineering \\ University of Louisville \\ Louisville, Kentucky
}

August, 2010 



\title{
ENHANCED CRYPTOGRAPHIC APPROACHES
}

FOR SCADA NETWORK SECURITY

\author{
By \\ Waleed H. ElSaid \\ A Dissertation Approved on \\ 07-02-2010 \\ by the following Dissertation Committee
}

Dr. James Graham, Chair

Dr. Adel Elmaghraby

Dr. Dar-jen Chang

Dr. Patricia Ralston

Dr. Rammohan Ragade 


\title{
ABSTRACT \\ ENHANCED CRYPTOGRAPHIC APPROACHES \\ FOR SCADA NETWORK SECURITY
}

\author{
Waleed H. ElSaid
}

07-02-2010

Due to the overwhelming increase in open source code, off-the-shelf software packages, third party and vendor codes, along with the ease of getting information about hacking network security systems and attacking the well known holes in security systems, the problem of having a secure network system is much more difficult than before this boom in technology and information broadcast. What makes the problem even worse is trying to secure a network for real time control, such as a network using supervisory control and data acquisition (SCADA) systems, because now the problem has two faces: securing the real time control system and at the same time keeping the response time of the system in the acceptable range for the transactions' level of service.

There is a strong trend to chose security frameworks that have been popular in the e-commerce sites of the web, particularly because they proven to be very mature and secure for more than one and half decades. Examples include the transport level security (TLS) and its predecessor secured socket layer (SSL) framework that is based on the very popular public key cryptography and key distribution algorithms, such as Rivest, Shamir and Adleman (RSA), elliptic curve cryptography (ECC), and Diffie-Hellman. 
Despite the fact that these algorithms proved to be very powerful against most types of attacks, they are not tailored to secure SCADA networks, and consequently cause a significant degradation in the performance time of real time transactions.

This dissertation offers two novel encryption algorithms for securing a SCADA network, the N-Secrecy and the Security Spectrum algorithms. N-Secrecy gave very good results when compared with the SSL; with N-Secrecy performance time in the range of one thousandth of the SSL. The Security Spectrum approach moved the encryption methodology from using numerical representations into using a physical representation based on modeling the conditions of the two communicating parties with a system of non-linear polynomials and then using computer algebra techniques. Both approaches have the potential to significantly enhance the security of commercial SCADA installations. 


\section{TABLE OF CONTENTS}

PAGE

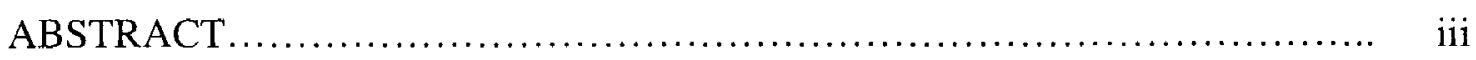

1. CHAPTER I: Introduction ............................................ 1

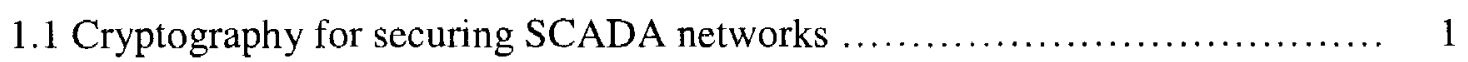

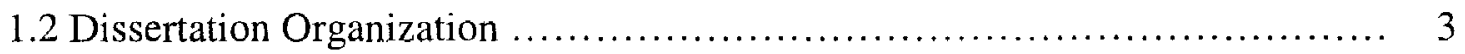

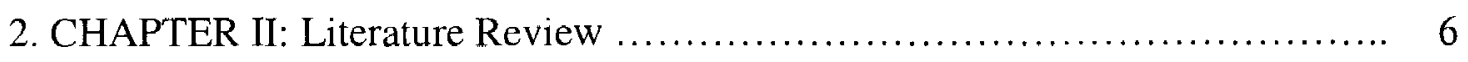

2.1 Introduction and typical usages of SCADA systems....................... 6

2.2 Importance of securing SCADA networks............................... 7

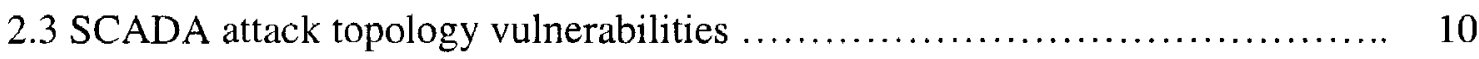

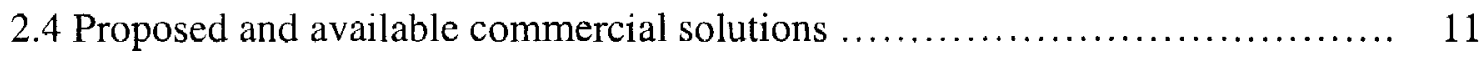

2.5 SCADA and IT systems ................................................ 13

2.6 SCADA in relation to cryptography and authentication $\ldots \ldots \ldots \ldots \ldots \ldots \ldots \ldots, 14$

2.7 Overview of Current Research ........................................ 16

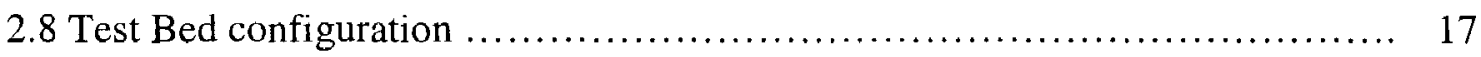

3. CHAPTER III: Enhancing SCADA security using multiple levels of secrecy .... 22

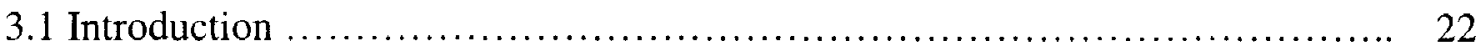

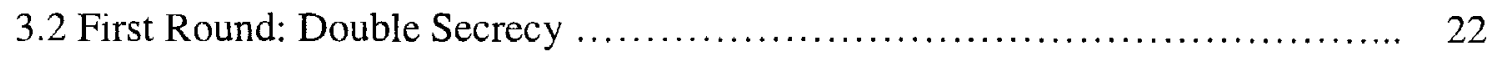

3.3 Second Round: Will it stand against various kinds of attacks?............... 24

3.4 Third Round: N-Secrecy Authentication Framework ..................... 26 
3.5 Final Round: N-Secrecy Authentication/Authorization Framework ........... 28

3.6 Binding the multiple Secrecy algorithms with real SCADA ................ 30

3.7 Multiple Secrecy algorithms and Other Authentication Frameworks ......... 32

4. CHAPTER IV: Prototype Implementation and Experimental Results .......... 34

4.1 Test bed configuration and Testing Methodology .......................... 34

4.2 Double Secrecy and N-Secrecy, Prototype Implementation ................ 35

4.3 Experimental Results for Double Secrecy .............................. 39

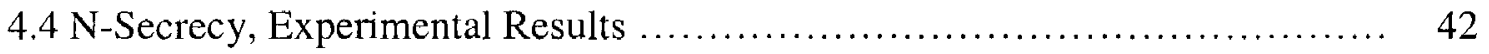

5. CHAPTER V: Computer Algebra techniques for Enhancing SCADA security .. 44

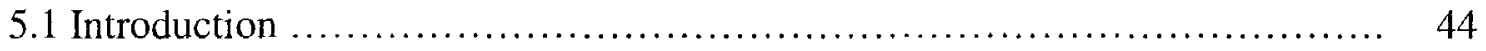

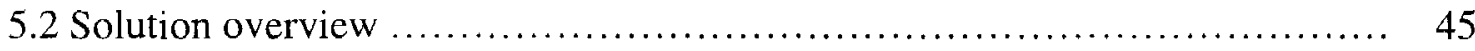

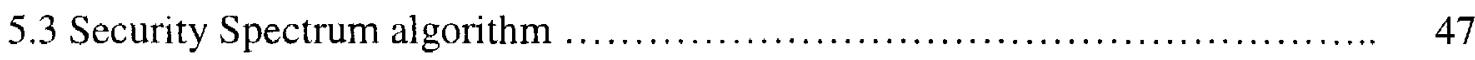

5.4 Using the Security Spectrum algorithm in a SCADA application ............. 48

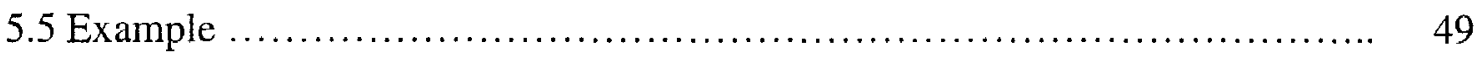

5.6 Security Spectrum and Diffie-Hellman ............................... 54

6. CHAPTER VI : Conclusions and Future Directions ....................... 57

6.1 Conclusions .............................................................. 57

6.2 Directions for Future Research ....................................... 59

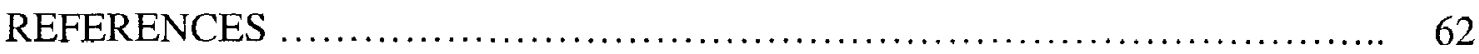

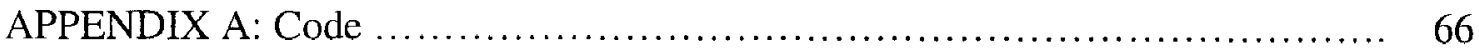

APPENDIX B: Algebraic Geometry and Computer Algebra concepts ............ 103

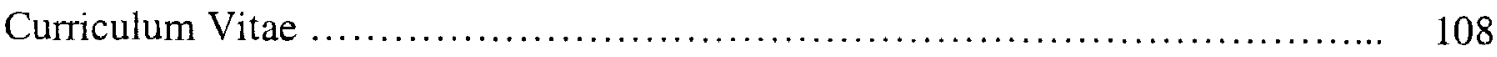




\section{LIST OF TABLES}

TABLE

PAGE

Table (4.1): Average and standard deviation of DNP3 messaging time with no security implemented, and with SSL implemented

Table (4.2): Average and standard deviation of DNP3 messaging time with different combinations of double secrecy

Table (4.3): Average and standard deviation of DNP3 messaging time with N-

Secrecy implemented using Triple-DES on large secret files using $N=3,4,5$, and $6 . . \quad 43$ 


\section{LIST OF FIGURES}

\section{FIGURE}

PAGE

Figure 2.1: A typical SCADA system network

Figure 2.2 Distillation Column and physical SCADA architecture ................ 20

Figure 2.3 Typical SCADA controlled distillation column ..................... 21

Figure 2.4 Federated SCADA controlled chemical plant $\ldots \ldots \ldots \ldots \ldots \ldots \ldots \ldots \ldots \ldots \ldots$

Figure 3.1: Double Secrecy authentication framework ........................ 24

Figure 3.2: Modified Double Secrecy authentication framework implementing the

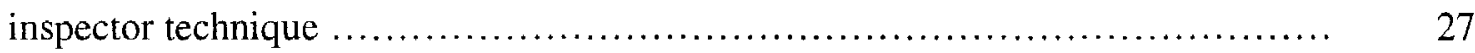

Figure 3.3: Message operation hierarchy example $\ldots \ldots \ldots \ldots \ldots \ldots \ldots \ldots \ldots \ldots \ldots . \ldots \ldots$

Figure 3.4 Double secrecy framework using distillation column testing bed ........ 31

Figure 4.1: Prototype implementation component diagram ..................... 35

Figure 4.2: No security implementation sequence diagram .................... 36

Figure 4.3: Double Secrecy implementation sequence diagram .................. 37

Figure 4.4: N Secrecy implementation sequence diagram .................... 38

Figure 5.1 Security Spectrum framework using distillation column testing bed ..... 49

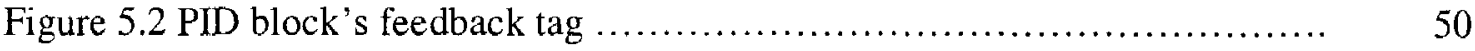

Figure 5.3 cascade control with Master/Slave PID blocks ...................... 50

Figure 5.4 Using security spectrum as the key generator algorithm and double secrecy

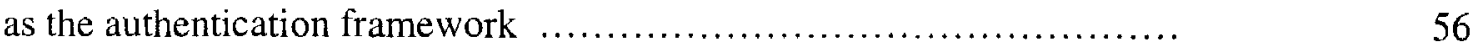

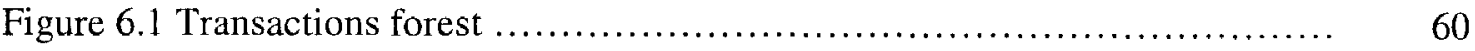




\section{CHAPTER I \\ INTRODUCTION}

The objective of this chapter is to present an introduction to the supervisory control and data acquisition (SCADA) network systems, and to the enhanced cryptographic approaches for SCADA network security, which is the subject of this dissertation. The introduction includes an overview of the usage of cryptography in securing SCADA networks and the objective and organization of the whole dissertation.

\subsection{Cryptography for securing SCADA networks}

SCADA is broadly used in many industries that require real time control systems such as, water management, traffic signals, electric power, oil pipelines, and manufacturing systems. The most famous and commercially available cryptographic approach that is adopted by many SCADA systems is the secured socket layer / transport level security (SSL/TLS) framework. Following is a brief discussion on what SSL/TLS is and what its advantages and disadvantages are when used with SCADA systems. The SSL/TLS framework involves establishing a mutual trust between each one of the communicating parties prior to initiating the communication. There are a few common public key cryptographic algorithms that can be used in order for each party to authenticate the other in the so-called SSL handshaking [27]. Below are brief details on some of those common cryptographic algorithms. Together with those public key cryptographic algorithms, the SSL also uses symmetric encryption algorithms like the DES, or triple-DES, and a message digest algorithm to validate the data integrity. 


\subsubsection{RSA}

The RSA algorithm works such that the first party uses two very large prime numbers that are a few hundred digits, $\mathrm{p}$ and $\mathrm{q}$, multiply them and send the product to the other party or parties to serve as the public key $k$. The other party uses $k$ to encode the message and sends the encoded message to the first party, which then uses its private numbers $\mathrm{p}$ and $\mathrm{q}$ to decode it. Hence, the idea is to use a one way function where $\mathrm{k}$ can be obtained from $p$ and q relatively easily but the inverse is not true [28].

\subsubsection{Diffie-Helman (Exponential Key Agreement)}

This algorithm depends on the choice of one prime number $\mathrm{p}$ and an integer $\mathrm{g}$ less than p. Those two numbers will serve as the public keys. The first party will have a private number of its own, a, computes $\mathrm{g}^{\mathrm{a}}$ mod $\mathrm{p}$ and sends the result to the other party which in turn will have a private number of its own, $b$, so it will compute $\left(\mathrm{g}^{\mathrm{a}} \bmod \mathrm{p}\right)^{\mathrm{b}}$ which is equal to $\mathrm{g}^{\mathrm{ab}} \bmod \mathrm{p}$. Now the other party will compute $\mathrm{g}^{\mathrm{b}}$ mod $\mathrm{p}$ and sends it to the first party which will compute $\left(\mathrm{g}^{\mathrm{b}} \bmod \mathrm{p}\right)^{\mathrm{a}}$ which is equal to $\mathrm{g}^{\mathrm{ab}} \bmod \mathrm{p}$ again. After this transfer and computation of numbers, both parties will have the same number which is $\mathrm{g}^{\mathrm{ab}}$ mod $\mathrm{p}$. This number then serves as the shared key between the two parties. Like the RSA Diffie-Helman algorithm depends on a one way function depending on the complexity of the discrete logarithm problem [29]. Elliptic curve cryptography (ECC) is similar to Diffie-Helman algorithm in that it depends on the discrete logarithm problem as well [30].

\subsubsection{Using SSL/TLS as the authentication framework in SCADA communication}


The real value of applying SSL/TLS in SCADA is that it is a well tested framework that is commonly used throughout the internet, and is much less expensive compared to adopting a new technology tailored to the SCADA networks.

The disadvantages on the other hand, are the facts that SSL/TLS is a very performance consuming framework because of the use of the non inverse functions that are not needed at all in the SCADA systems because the communication will be between two SCADA units that actually know each other, not like the communicating parties over the internet.

\subsection{Dissertation organization}

This dissertation is divided into six chapters and two appendices. The second chapter gives a literature review on the SCADA network systems, the importance of securing those systems, a comparison between SCADA and IT systems, the SCADA attack topologies, available solutions, and the authentication role in securing SCADA systems. Chapter III then establishes the core of this dissertation which is a novel cryptographic framework for securing SCADA networks in a manner that is less computationally expensive than using the secured socket layer (SSL). The chapter starts with a preliminary version of the algorithm called Double Secrecy, and then discusses a variety of possible attacks, presenting solutions by extending the algorithm. Afterwards, the algorithm is extended to the N-Secrecy to offer flexibility administering the piece of information being transferred back and forth through the SCADA networks by defining the number of secrets required according to the vitality of this information, and then the whole authentication idea is extended to the idea of authorization to give the N-Secrecy another dimension. Chapter III then presents another version of the N-Secrecy algorithm 
that is tailored to the test bed available using the Chemical Engineering Department distillation column, and concludes with brief comparisons between the multiple secrecy frameworks and a few authentication frameworks.

Chapter IV presents the testing methodology and the testing results of the Double Secrecy and the N-Secrecy algorithms. The chapter starts with detailing some information about the way the code is designed and the way it is tested and compared to the baseline, which consists of three versions of the SSL, depending on whether the encryption algorithm used is the Rivest, Shamir and Adleman (RSA), Diffie-Helman, or elliptic curve. The data presented in this chapter covers the following variables: the length of the secret being transferred in the message; the way the inner encryption inside the double secrecy is implemented whether it is DES or Triple-DES, and the three ways of implementing the SSL. Chapter IV then switches the gear to presenting the experimental results of the N-Secrecy, fixing the inner encryption algorithm used to the Triple-DES and varying two variables; the length of the secret being transferred in the message, and the number of secrets used. Chapter V commences with presenting a different type of problem that is the key distribution problem, and how current algorithms like Diffie-Helman deal with that problem, and propose a new algorithm using computer algebra techniques. First, a brief discussion of the new algorithm is presented, then issues are found and discussed and afterwards solutions are offered to those issues, and finally, the new algorithm named the Security Spectrum is presented in section 5.3. The new algorithm is then presented using the notions of the SCADA distillation column test bed offered by the Chemical Engineering Department. Chapter VI provides conclusions and directions for future research. All the references that are used in throughout the 
dissertation are given in the References. Appendix A contains the code created to implement the $\mathrm{N}$-secrecy and the security spectrum algorithm together with the code for the RTU, MTU, and the SSL. Appendix B covers some mathematical concepts used mainly in chapter V like Grobner bases, ideals, varieties, and polynomial ring theory. 


\section{CHAPTER II}

\section{Literature Review}

This chapter presents a literature review on the subject of securing SCADA networks. The emphasis is on the authentication problem, which involves how two units, typically the RTU and the MTU, authenticate each other prior to establishing communications. The literature survey presented includes the following topics: (1) an introduction to the SCADA architectural differences and typical usages of SCADA systems; (2) discussion of the importance of securing SCADA networks; (3) discussion of SCADA attack topology vulnerabilities; (4) proposed and available commercial solutions; (5) SCADA and IT systems; (6) SCADA in relation to cryptography and authentication; (7) an overview of current research; and (8) discussion of a SCADA test bed developed at the University of Louisville.

\subsection{Introduction, SCADA architectural differences and typical usages}

SCADA systems were developed due to the need to control and monitor real-time processes, like those in oil pipelines, chemical and physical plants, and so forth. These systems replace human monitoring and promote monitoring at the level of the whole system and also facilitate remote control from a central location called a master terminal unit (MTU) [20]. SCADA systems were designed originally to be reliable and easy to use with very little concern about communication security. The functions of SCADA systems processes usually require on the order of seconds or tens of seconds to complete.

SCADA architectures can be configured using any of the following communication 
options [20]: point-to-point, where there is a link between each RTU and the master unit; series communication, where each RTU is connected to the closest RTU in its neighborhood and so forth until one of the RTUs links directly to the master unit; seriesstar communication, where a certain RTU simulates a hub in its neighborhood and all the remote units in that neighborhood links to it; multi-drop communication architecture, where there is like a message bus from the master unit and each remote unit subscribes, and connects itself to that bus [31]. Connections to remote devices can be made over dial up, Ethernet LANs, leased lines, or SCADA radios. Some of the operations conducted in SCADA systems can be processed asynchronously like pulling values in a background job to be used in generating reports later, and some of the operations need to be conducted in real-time like some control transactions for aviation systems. Following is a figure showing a typical example of SCADA communication links according to the technical report of the American gas association (AGA) published in 2006 [20].

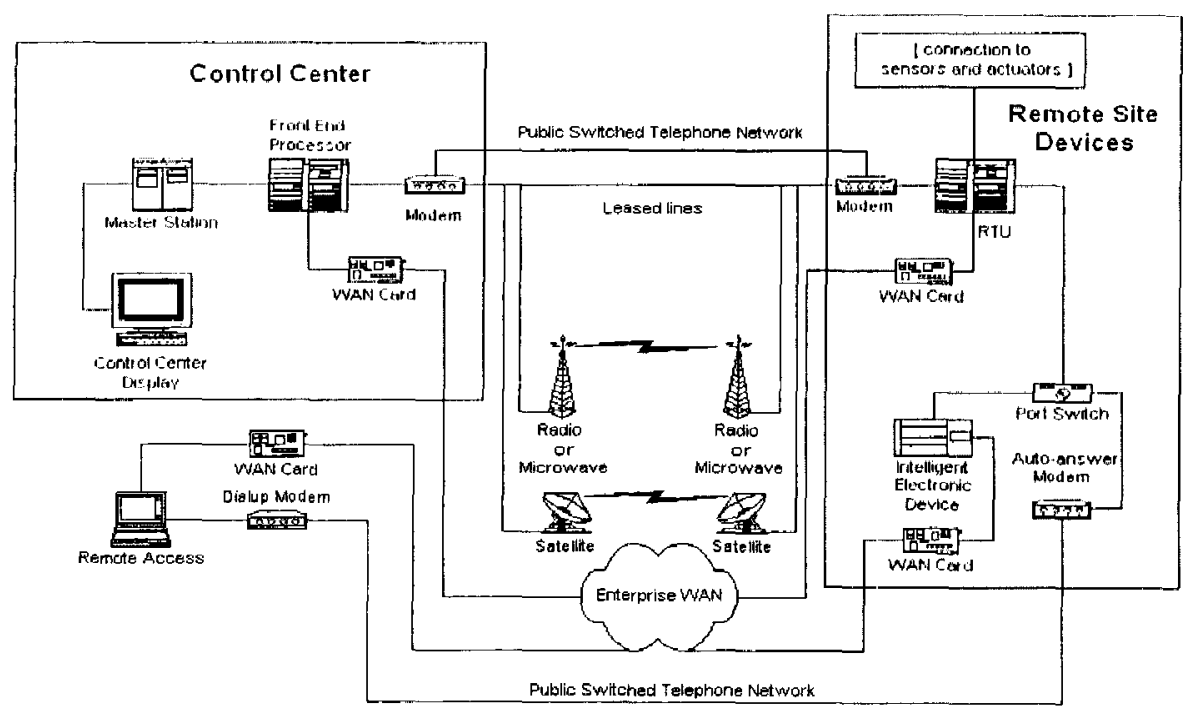

Figure 2.1: A typical SCADA system network (adapted from AGA [20])

\subsection{Importance of securing SCADA networks}


The US Department of Energy states that automated control has helped to improve the productivity and reliability of energy systems by improving the performance of the real time transactions and decreasing the backup time [1]. The vulnerability of SCADA networks to cyber attacks has increased remarkably, since early SCADA designs did not consider the security risks that arose due to the switch from private and tailormade software, operating systems and telecommunication networks to more general and open source software, and increasing the use of Internet connected networks [1]. The National Research Council has identified the security of SCADA systems as one of the fourteen most important initiatives in making the nation safer [2].

The Committee of Science and Technology for Countering Terrorism, of the National Research Council declared the vulnerability of the power grid's control systems to cyber attack as a significant challenge in protecting the electric power grid [2]. According to the committee, the special problem that should be reviewed in securing the SCADA systems is the way data is transmitted between control points in the SCADA network. Encryption techniques, enhanced firewalls, and cyber intrusion-detection technologies should be used to improve security and reduce the potential for hacking and disruption [2] and [5]. Existing surveillance technologies that were developed for defense and intelligence applications should be investigated for their usefulness in defending against terrorist attacks [2], [5], and [6].

The National Institute of Standards and Technology (NIST) defined a strategy named defense-in-depth, to protect industrial control systems (ICS), to which SCADA systems belong. This strategy stated that a typical ICS should begin developing security policies, procedures, and educational material that apply specifically to ICS, and that ICS 
security policies and procedures should be based on the Homeland Security Advisory System Threat Level, deploying increasingly heightened security postures as the Threat Level increases. According to the NIST, security should be addressed throughout the life cycle of the ICS from architecture to procurement to installation, to maintenance, to decommissioning, and network topology should be implemented for the ICS having multiple layers, with the most critical communications occurring in the most secure and reliable layer. Also NIST recommended that a logical separation between the corporate and ICS networks should be provided [16].

NIST further recommended that a demilitarized zone (DMZ), which is the area between two firewalls, network architecture should be employed to prevent direct traffic between the corporate and ICS networks. Critical components on the other hand, should not have a single point of failure. Critical systems should be designed for fault tolerance to avoid catastrophic events and in addition, systems should be designed to fail securely. Unused Internet protocols (IPs), ports and services on ICS devices should be disabled after testing so that not to compromise ICS operation. A role-based access control should be implemented, that is to make the ICS networks, devices, and services' physical access as well as user privileges restricted to only those required to perform specific jobs [16].

According to NIST, ICS networks should have a different authentication paradigm and credentials than those of the corporate networks. Finally, security controls such as anti-virus software and intruder detection systems (DS) should be implemented to prevent and detect the introduction and accumulation of malicious software to the ICS. Security techniques such as encryption of ICS communications and data storage should be applied. In addition, human monitoring and administration should be there all the time. 


\subsection{SCADA attack topology vulnerabilities}

Existing SCADA protocols were designed to use a rather simple error detection paradigm like the use of cyclic redundancy codes (CRC), parity checks or similar technology. The sender of the message will calculate the CRC and append it to the message. The receiving device, on the other side, will calculate the CRC for the message and compare it to the value received with the message. If a bit was flipped during transmission, the $\mathrm{CRC}$ indicates a transmission error. Another common characteristic for SCADA protocols is the fact that they were not designed with built in authentication or validation services, assuming a level of implicit trust. For example, when a message is received by an RTU, the source of the message is checked, and if that source is known, the request is enacted. Furthermore, DNP3 is becoming the standard protocol in the electric distribution world, and DNP is an open standard, with published information regarding message structure and vulnerabilities appearing on the Internet.

The ways critical infrastructure can be attacked can be categorized to the following three modes. The attack can be the classical physical destruction attack to a critical control or data center; it can come through the communication wires, or through a compromised trusted party in the network [21]. Most of the currently reported incidents have an internal origin. Lack of authentication and mixed roles usually result in granting non-competent users access to critical functionality. On the other hand, the human machine interface (HMI) helps facilitate attacks by allowing interactions with the system from a higher level without the need to understand the underlying process. This kind of visibility, along with the fact that SCADA protocols have no authentication does much of the work of the attacker. On the other hand, the huge amount of available information 
published on SCADA infrastructure components allows an attacker to discover new vulnerabilities at a lower level.

Another important fact regarding electric distribution SCADA systems is that they are geographically dispersed. Some of the SCADA infrastructure components, such as RTUs or programmable logic controls (PLCs) are usually located in remote locations, physically dispersed from the central unit, and gaining physical access to the facilities that house these remote devices is usually trivial. Although these units are physically remote to the central unit, they are connected to the SCADA system (and potentially to other corporate networks) logically, offering an attacker a point of entry to an apparently isolated network. On the other hand, connections to remote devices can be made over dial up, leased lines, or SCADA radios. Each of these communication methods can be compromised. For example, SCADA radio is a strong signal, typically one watt. At that power, the signal can travel more than ten miles providing a significant opportunity for an adversary to break in without being detected [3]. One final point to mention is that SCADA systems are evolving towards commercial platforms and open protocols (PCs, $\mathrm{TCP} / \mathrm{IP}$, etc.) as time passes which adds the traditional vulnerabilities of these technologies, like worms, viruses and other malwares on top of vulnerabilities particular to the SCADA environment [8].

\subsection{Proposed and available commercial solutions}

\subsubsection{Proposed solution on the process level}

Most of the literature published on SCADA security addresses the issue from the process perspective and a very few consider the issue from the technical perspective. Less detailed articles and white papers have been published to educate company executives 
about SCADA systems, such as "SCADA Security-Advice for CEOs" [11], that summarizes some of the issues of which company executive should be aware. Examples include separating the SCADA network from the Internet and the corporate network. This separation is critical to risk management in modern SCADA systems. Network architecture weaknesses can significantly increase the risk from Internet and other sources of intrusion.

One major issue to be handled is confidentiality. SCADA systems were not designed with security specifications, so there is no standard protocol having built in authentication. If lower level protocols do not provide this confidentiality then SCADA transactions are communicated with no authentication, meaning that intercepted communications may be easily read. Authentication is another very important issue to keep in mind. Many SCADA systems give little regard to security, often lacking the memory and bandwidth for sophisticated password or authentication systems. As a result, there is often no mechanism to identify, authenticate or authorize a system user. Due to its nature, SCADA systems are mostly stateless, like those of banking ATM machines, lacking a session structure which, when combined with the lack of authentication, allow the injection of malicious requests or replies into the system without any prior knowledge of what has gone on before. The "SCADA Security-Advice for CEOs" article also gives a brief on where the threat is [11]. Following are some examples of threat sources: (1) insider attack from employees or ex-employees; (2) organized crime driven by financial incentive to penetrate SCADA systems; (3) mistakes made as a result of lack of training, or an oversight; (4) terrorists, and (5) generic Internet threats such as worms, Trojan horses and viruses that infect systems on the Internet can also affect SCADA systems 
when they use the same software and protocols. Many of the intrusions may not be the result of a deliberate attack. SCADA systems may be infected merely because there will always be hackers and virus writers challenged and fascinated with technology.

\subsubsection{Proposed solution on the technical level, available commercial solutions}

Most of the SCADA solutions that are commercially available now have recently incorporated some authentication techniques, such as the SEL-3332 Intelligent Server, relays, remote $1 / O$ modules, and the SEL-2411 programmable automation controller,

which uses the following to manage cyber security [7]: Encrypt Ethernet-based engineering access and SCADA protocols with SSL/TLS; Protect VPN access with IPsec link security; and Manage access via user accounts.

\subsection{SCADA and IT systems}

There are two kinds of operating systems in industrial control systems. One of them uses Windows or UNIX, each of which has role-based security to authorize the user and direct him to the right information according to his role. The second one is the control processor software itself that actually retrieves and sorts the data and sends commands with no means of authentication or authorization [23].

There are several differences between SCADA systems and regular IT systems making securing the SCADA systems a much harder job than securing the regular IT systems. A typical workstation or network will run an operation until it is finished. The real time control system on the other hand will prioritize all the operations in its queue and will process them accordingly. Whenever a new operation enters the queue, the prioritization is repeated, meaning that each time a higher priority transaction appears in the queue it will stop what it is doing. Another important difference is that IT systems 
upgrade the hardware regularly with no problems. SCADA field devices on the other hand, often use inexpensive, low cost microprocessors. Some electrical devices in the industry are still using the 8088 processor introduced in 1978, despite the fact that only the 486 and later processors can use the encrypted authentication schemes with no unacceptable delays [23].

\subsection{SCADA in relation to cryptography and authentication}

The Government Computer News (GCN) published in January, 2005 on its web site that a SCADA encryption appliance is soon to be commercially available. This product, called Datacryptor, was designed to be installed between the remote device and the management console, and it was supposed to support both Modbus and DNP protocols. The Datacryptor was to use the advanced encryption standard algorithm to encrypt on-going messages and authenticate the administrators as well via a password and a security token. It is well conceived that encryption would add latency on the performance of regular SCADA; the GCN though, said that the Datacryptor would keep this latency less than $20 \%$ and this should be acceptable [22]. The Datacryptor is now available commercially.

All of the previous discussion points toward authentication as an important partial solution of current SCADA security problems. The Pacific Northwest National Laboratory (PNNL) adopted a project for designing and developing a novel SCADA communications authenticator technology, funded by the U.S. Navy. The idea behind the new protocol, called Secure SCADA Communications Protocol (SSCP), was to wrap the original SCADA communication traffic with a unique identifier and an authenticator [9].

The SSCP then uses the authenticator to authenticate the unique identifier in the 
wrapper to validate the communication, and consequently detect various attack scenarios, including man in the middle, injected traffic, or message replay. The SSCP is to be available as an embedded software solution running on the SCADA master or input/output server. The authenticator technology directly supports the Roadmap to Secure Control Systems in the Energy Sector [12], with milestone targeting widespread implementation of methods for secure communication between remote-access devices and control centers.

In terms of the Department of Defense (DOD) technology readiness level definitions [13], SSCP has currently achieved level seven (i.e., system prototype demonstration in an operational environment). The goal for this project is to move the SSCP toward technology readiness (level eight), where the technology has been proven to work in its final form and under expected conditions. Comprehensive testing will be performed to confirm that the technology will fulfill its technical objectives when deployed under a variety of expected conditions in the field. The goal is to facilitate earlier industry adoption of a novel security technology that is well suited for securing control systems used by energy infrastructures [14].

Donald Wallace [15] stated that the open nature of the Internet requires careful consideration of data security measures when implementing Internet-based SCADA systems. Processes, procedures, and tools must be put in place to address availability, integrity, confidentiality, and protection against unauthorized users. Regarding availability, system up time must be maintained at the highest levels through use of redundant servers, so that there is no single point of failure. Firewall protection must be provided in the Gateway and servers along with automated monitoring to detect DNS 
attacks. Regarding integrity, the system must ensure that the data is not modified or corrupted through the use of encrypted data signatures.

As for confidentiality, the system must ensure restricted access to data through use of encryption, and to the system by employing authentication frameworks such as SSL. Regarding protection against unauthorized users, it is realized that multi-layered password protection must be provided at all levels in the system. In other words there should be no single sign on (SSO) in SCADA systems.

Much of the literature, discussing the need for SCADA authentication, mentions SSL/TLS as an off-the-shelf solution [3,7]. Concerns using the SSL/TLS as a well developed Internet technology in authenticating private network systems like the SCADA arise, as why would public keys be used in a private network and getting the extra overhead of using an intensively computing irreversible function to encrypt data. SCADA systems were designed primarily to enhance communication between control systems and security should not come with the price of sacrificing the performance.

\subsection{Overview of Current Research}

One of the very interesting initiatives on which research is currently being conducted is the SCADA Honey Net Project conducted by Cisco Systems, Inc [9], which is about building honey pots for Industrial Networks. The goal of this project is to determine the feasibility of building a software-based framework to simulate a variety of industrial networks such as SCADA architecture. There are several uses for this project: (1) gathering data on attacker methodologies and tools, so that data mining can be done later on this data to gain information on attacker trends; (2) testing a live protocol implementation; and (3) developing research countermeasures, such as device hardening, 
stack obfuscation, and reducing application information [9].

Aside from simulating the real SCADA systems, the other important objective of the honey net project is to conduct research and eventually block all cyber attacks on SCADA systems, the group in the SCADA Safe project proposed a new protocol for link encryption and integrity checking of SCADA messages passed over slow serial lines [10]. They have also started an implementation project for that protocol, and they named it the SCADA Safe project. That serial SCADA protection protocol (SSPP) is for cryptographically protecting existing serial-based SCADA communications. A device that speaks SSPP is known as a SCADA cryptographic module (SCM). A SCADA message received from a SCADA master or remote unit on SCM plain text port will be protected and sent out to the SCM cipher text port as an SSPP message. An SSPP message received on a SCM cipher text port will be verified, decrypted, and sent out to the SCM plaintext port. SCM devices are deployed between SCADA devices and communications links modems. The key point here is that these devices must assure is data integrity, that is the commands and responses are not forged or altered during transmission.

\subsection{Test Bed configuration}

This section gives a brief discussion on the test bed that is going to be used for testing the suggested cryptographic frameworks. First, the test bed that is available in the Department of Chemical Engineering is presented and then a brief look at real SCADA controlled network systems is presented.

The test bed consists of an operating 14 foot, 50 liter, 6 -tray distillation column. Data collection as well as distillation process control are carried out using actual SCADA 
hardware and software. The system consists of the distillation column, two temperature sensors, five flow sensors, two level sensors, and five flow control valves as shown in figure 2.2 below. The distillation column separates a $20 \%$ molar solution of methanol and water into a distillate with concentration high in methanol (tops product) and a residue, which is mainly water (bottoms product).

The distillation process, from start up to shut down is monitored and controlled by a computer-based SCADA control system, specifically iFIX software from GE-Fanuc [25]. A digital to analog and analog to digital terminator panel links the sensors and actuators to the computer serial port. The distillation column is a MIMO (multiple input-multiple outputs) process with nine variables that can be controlled: distillate accumulator and reboiler levels, top and bottom temperatures, and distillate, reflux, bottoms, feed and steam flows. There are nine proportional integral derivative (PID) controllers for these variables. PID controller tuning parameters and controller set points can be changed.

The time required from start-up to achieve stable operation takes about 3 hours. The system can be monitored throughout operation and changes can be made to set points during this time before reaching the desired operating state. Remote monitoring and control allows a control engineer or operator to use the University's LAN as a control network and monitor and control the distillation column from a nearby office.

Remote monitoring and control are to be achieved using the HTTP protocol and a Tomcat web server on the computer connected directly to distillation column sensors and valves controllers. A computer on the LAN can then connect to the distillation column control page that provides an HMI interface, pull data values from the server and update the values of control variables by sending them from the client back to the server. 
The entire system can be viewed as a SCADA system where the iFIX software on the lab PC collects data from the sensors, makes local control calculations, and sends control signals to column hardware. The laboratory PC also serves as an RTU for multiple MTUs and the University LAN provides the network connection between the RTU and MTUs [24].

Interaction between the iFIX software and the tomcat web server is to be achieved through two files, INPUT.DAT and OUTPUT.DAT. Process variables are placed in OUTPUT.DAT by iFIX and iFIX reads the value of certain control variables from INPUT.DAT.

\subsubsection{How this testing bed differs from actual SCADA-controlled distillation column used in industry,}

This test bed environment differs from commercial chemical manufacturing environments. However, the remote monitoring and control are similar. According to the research done, the following categories of different SCADA-controlled distillation columns, have been found 


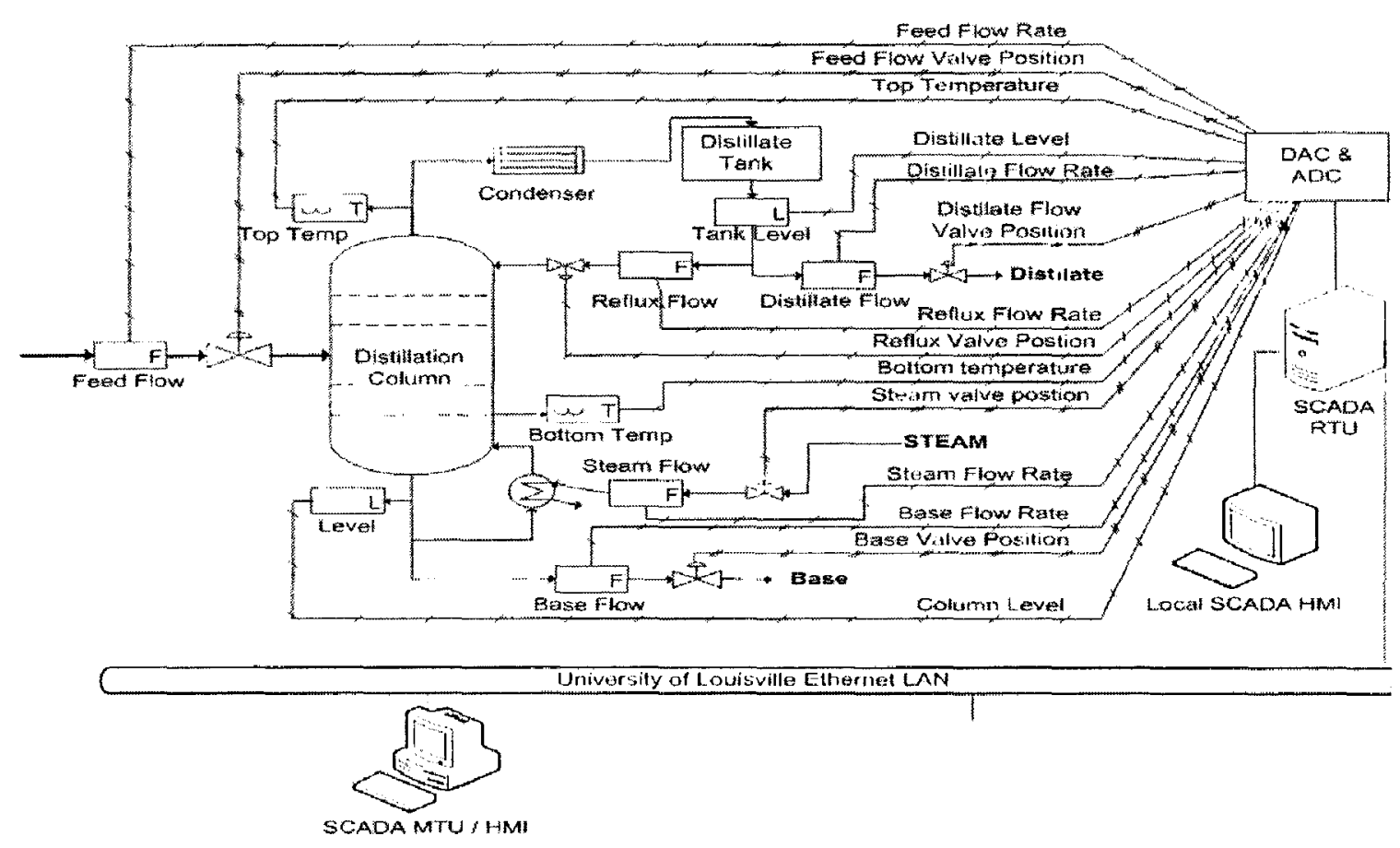

Figure 2.2 Distillation Column and physical SCADA architecture adapted from [24].

1. Plant is monitored and controlled with some SCADA software, like the iFIX used in the above mentioned test bed. However, in our test bed there are digital to analog and analog to digital terminator panels to link the sensors and actuators to the computer via serial ports. Whereas, in those actual SCADA controlled distillation columns, plant data is collected via a hub of TRIO (Taylor Remote VO) and sent back to the controller over a high-speed data highway. Custom graphics and information pages provide the user interface to the system. They might contain information module system (IMS) nodes to archive all plant data. They might also have Ole for Process Control (OPCs), to provide connectivity to third party software packages for web based plant-monitoring, simulation and reporting, see figure 2.3 below. 
2. Some chemical plants have multiple processes units that are connected to a specialized hardware interface. Each specialized interface is then connected to a central PLC that works as a mediator (hub) and proxy to SCADA software on a central server connected to a LAN of HMI interface workstations, see figure 2.4 below.
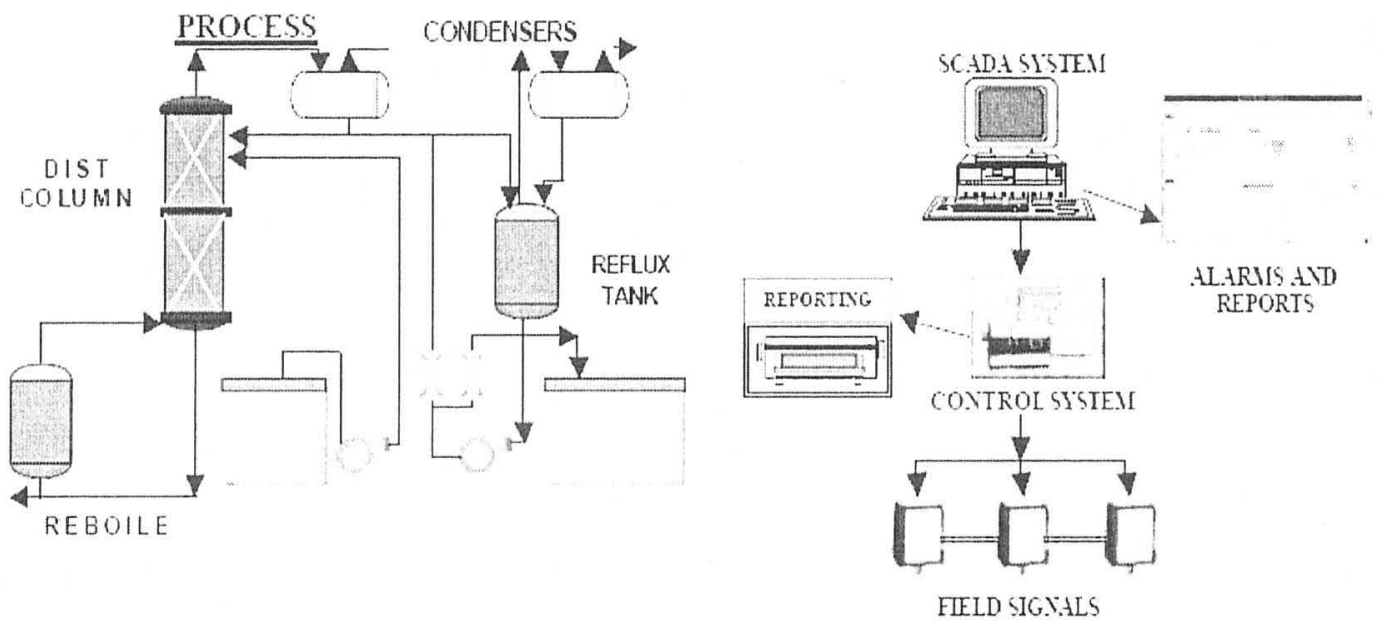

Figure 2.3 Typical SCADA controlled distillation column adapted from [18]

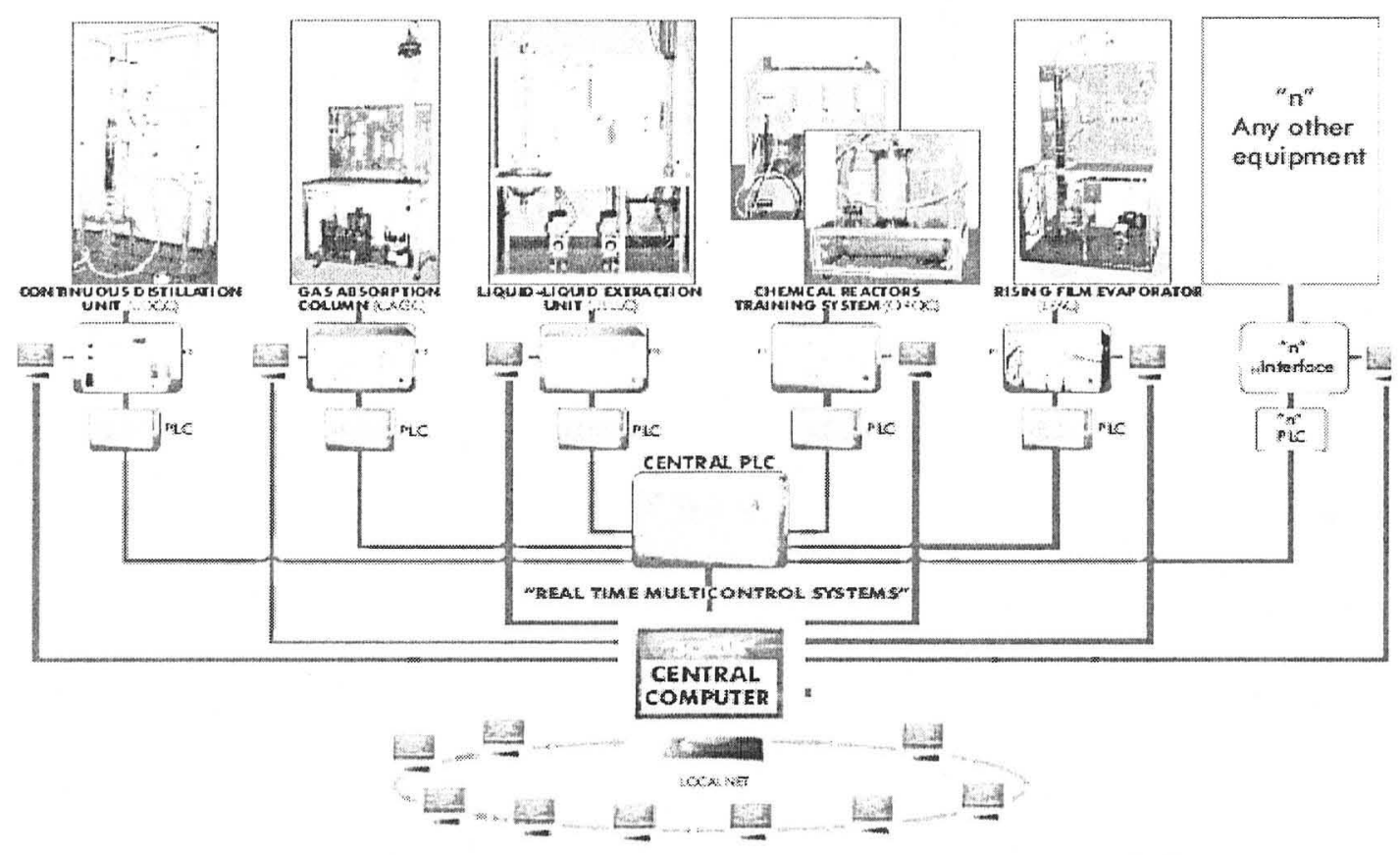

Figure 2.4 Federated SCADA controlled chemical plant adapted from [18] 


\section{CHAPTER III}

\section{Enhancing SCADA security using multiple levels of secrecy}

\subsection{Introduction}

As mentioned in the literature, SCADA systems were designed primarily to handle the data control and acquisition in an interactive mode. Performance was the main issue in designing SCADA systems, and security was completely neglected because all the operations were being conducted in a private network, where security is irrelevant. Now, being operated in many private networks connected together using public open networks, the security becomes a crucial issue. Many techniques have been adopted especially for SCADA, as well as tailoring the proven good techniques such as TLS/SSL. These techniques however, were either very heavy and performance consuming, or not secure enough to certain kinds of attacks.

Hence, the problem can be summarized as how to develop a well-secured framework for SCADA. This framework should be constrained to the SCADA protocol, typically the DNP3. This framework should be very light in terms of computational complexity, so as not to sacrifice the performance on behalf of security. This framework should stand against all kinds of attacks. Finally, this framework should vary the level of security according to the level of threat.

\subsection{First Round: Double Secrecy}

The idea is to keep two private keys, two secrets, and a hash table at each unit as follows: 
1. The general-purpose private key (GPK) between the MTU and all the RTUs that the MTU communicates,

2. A specific private key (SPK) between the MTU and each RTU,

3. Two secrets between the MTU and each RTU, or each pair of sender-receiver (SSEC1 and SSEC2), and

4. A hash table containing Key-Value pairs list that corresponds to a list of SSEC1(SPK, SSEC2), i.e. Key is SSEC1, and value is (SPK, SSEC2).

To send a message from the MTU to the RTU or vice versa, the following authentication scenario happens (figure 3.1),

1. The header of the message will be the secret shared between the sender and the receiver (SSEC1) encrypted using the GPK,

2. The message body will contain the actual message encrypted using the SPK,

3. The footer of the message will be SSEC2,

4. The message will be sent to the receiver,

5. The receiver will decrypt the header using the GPK,

6. The receiver will use its own hash table to look up the decrypted message header,

7. If the hash-table lookup process results in Null, this will mean an authentication failure. Since, this means that the encrypted header was not SSEC1 at the first place,

8. If the hash-table lookup process succeeds, this will mean that the header is SSEC1, which is then used to get the SPK, and SSEC2,

9. The SPK will then be used to decrypt the footer of the message, 
10. If the value of the decryption equals SSEC2, then the message is authenticated, and

11. SPK will then be used to decrypt the message.

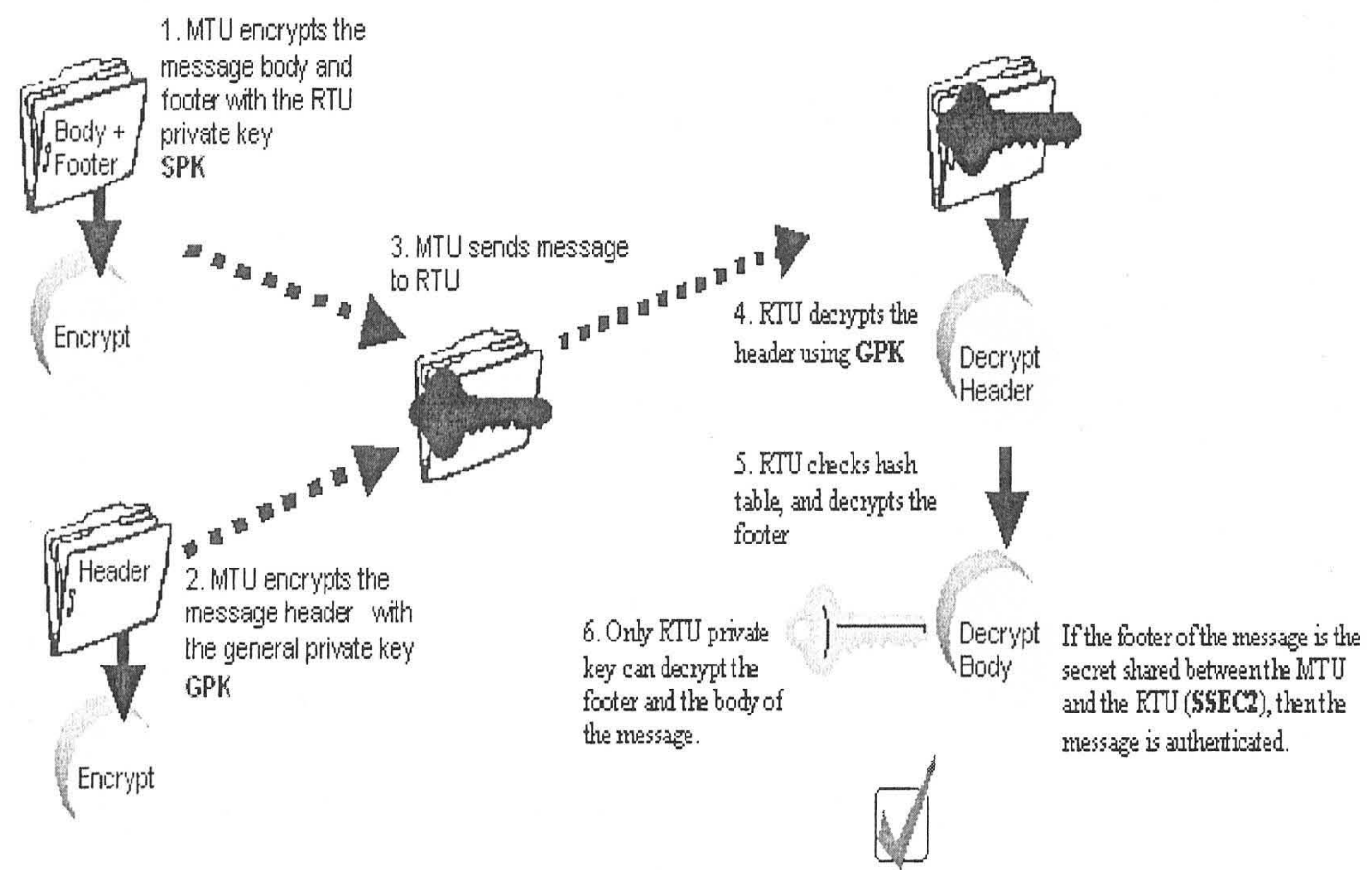

Figure 3.1: Double Secrecy authentication framework

\subsection{Second Round: Will it stand against various kinds of attacks?}

The objective of this section is to determine whether the double secrecy authentication pattern for protocol security can avert the denial of service (DOS) attacks. Apparently, the pattern is working fine for all other kinds of attacks like the spoofing, and man in the middle attack, since it depends on the hash table the two secrets and two private keys which makes the pattern secure in case of message interception and the spoofing. DOS attack happens when false messages are sent to the terminal unit, which has no problem in identifying that those are false messages because of the authentication 
technique used, but the problem happens when those false messages are sent to the terminal unit in such a high frequency that the unit spends so much time processing the authentication technique before it figures out that they are false, and eventually the unit becomes overwhelmed and denies the service to real messages.

The classic solution to this problem is to use some kind of a proxy to block out the IP address causing those false messages. Now the real problem comes with using the Spoofing-DOS attack, that is when some intruder gets inside the system of a real terminal, and sends false messages, because at this time the IP blocking will simply block the real terminal that is being spoofed, and whenever the spoofing ends and the spoofed terminal tries to send real messages it discovers that the messages cannot be delivered because the IP is blocked.

Using Double-Secrecy, the key point is that there are two testes for the message to be authenticated: first the header of the message should be a key in the receiver terminal hash-table after being decrypted by the GPK, and secondly, the footer should be the SSEC2 (from the hash-table) after being decrypted by the SPK. The loss of the processing time comes because the receiving unit will wait to receive the whole message and then start processing the first test to know it is a false message, or a potentially authenticated message. Hence, a kind of inspector component is needed to take the first packet of the header when it is received and do some pattern matching to check if this packet is promising to be a part of some key in the hash-table or not. That way, false messages will be discovered from the very first packet, and whenever, a message header is a promising one, it will be forwarded to the terminal to proceed with the two testes (figure 3.2). 
However, three problems arise: (1) sometimes one packet is not enough to block most of the false messages and keep them from over loading the terminal; (2) the inspector component itself can get loaded and the same problem of loading and getting out of service happens with the component, which will then lead to DOS state of the whole unit; and (3) a major problem is that encryption/decryption functions need not be linear, in fact almost all of them are not, in a sense that " $F(p q) \neq F(p) F(q)$ ", which means that even if the message is not false this does not mean that the decryption of the first packet of the header will be the first packet of the decrypted header.

Those problems can be addressed like this: (1) more than one packet can be used according to the configuration of the terminal to decide whether the message is false and the sender should be blocked or not; (2) a vector of inspectors can be used with some sort of a master inspector component that acts like a load balancer to prevent the inspector component from getting over-loaded, in a sense that whenever some inspector component becomes almost loaded, the master inspector will create another inspector component from the pool of available inspectors, and put it in service. If it happens that all the inspectors in the pool are in service, the master will simply block this IP until an inspector becomes free, but this will be for a very short period of time comparing with the IP blocking technique; and (3) to solve the linearity problem, the key should be reconstructed in the hash-table so that instead of being SSEC1, it can be the result of concatenating SSEC1 (first packet) with SSEC1 (rest of the packet).

\subsection{Third Round: N-Secrecy Authentication Framework}

The objective of this round is to make the level of security on the SCADA systems protocols vary on demand, that is initializing it on level $\mathrm{L}$ and increasing to L2 in 
case of sending more vital messages, or detecting an increase of the threat level, and further increase it, say to level L10, in case of being under severe attack. Previous sections introduced a novel framework for authenticating the communication between two parties depending on two secrets, and two private keys.

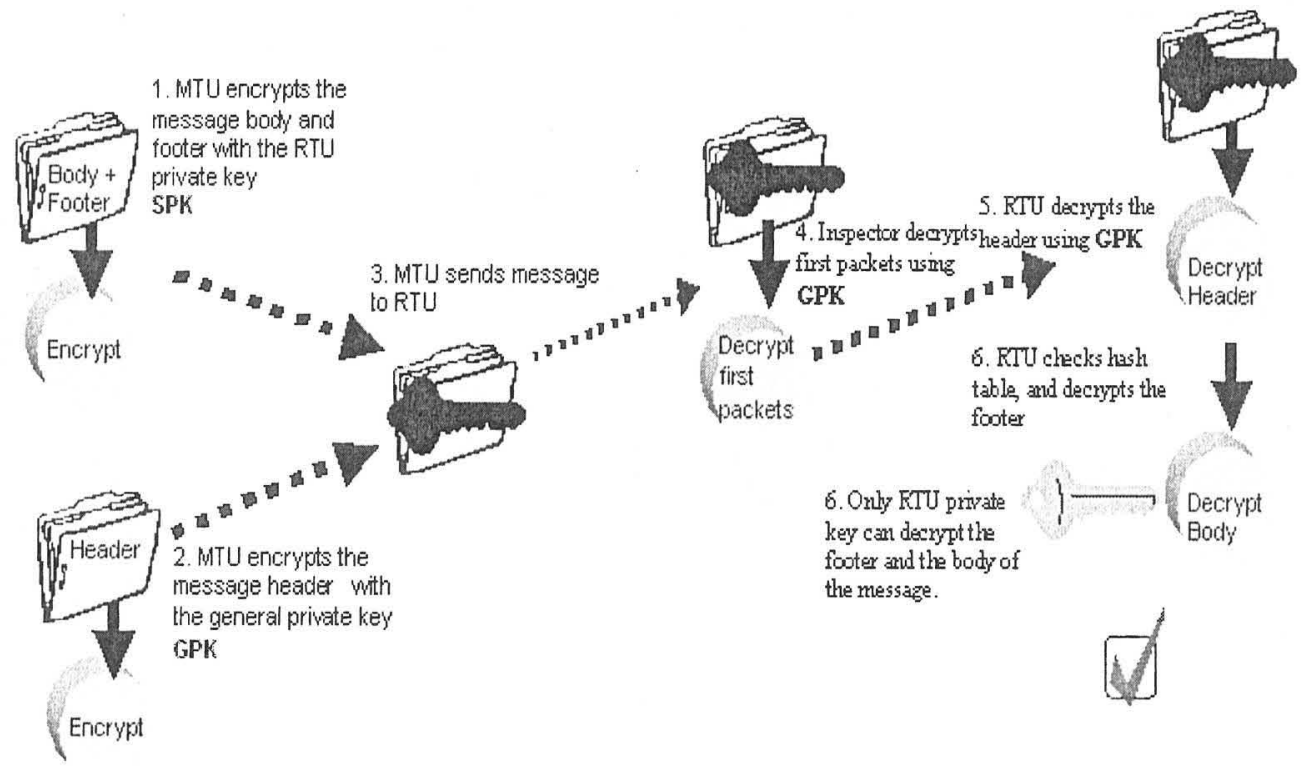

Figure 3.2: Modified Double Secrecy authentication framework implementing the inspector technique

The idea here is to generalize the double secrecy authentication framework to use $\mathrm{N}+1$ secrets instead of two. The exact algorithm is as follows:

1. The header of the message contains the secret shared between sender and the receiver (SSEC0) encrypted using the GPK,

2. The footer of the message contains $n$ secrets, where $n>0$,

3. The message body contains the actual message encrypted using the SPKn,

4. The message is sent to the receiver,

5. The receiver decrypts the header using the GPK,

6. The receiver uses its own hash table to look up the decrypted message header, 
7. If the hash-table lookup process results in Null, this would mean an authentication failure,

8. If the hash-table lookup process succeeds, this will mean that the header is SSEC1, which is then used as a key in the hash-table to give a vector of pairs (SPKi, SSECi); $\mathrm{i}=1 . . \mathrm{n}$,

9. The length of those secrets is known for both the sender and the receiver,

10. An iteration is being done to read the SSECi length number of bytes of the footer, decrypt them using SPKi, and compare it to the SSECi,

11. If the comparison succeeds, then the index $i$ is incremented, to read the next one,

12. If comparison fails at any stage, then the sender is not authenticated,

13. SPKn is then used to decrypt the message.

\subsection{Final Round: N-Secrecy Authentication/Authorization Framework}

Since authentication only is not enough to undergo vital procedures that messages might have, like unlocking dams or cutting circuits, another level of security, which is the authorization, is introduced. Authorization checks with other administration master units to verify if this master unit is authorized to request such procedures. This authorization workflow should again be dependent on the level of importance of that operation. Hence, in this round, both of those frameworks are mixed up together to make the level of authentication more generic depending on $\mathrm{N}$ secrets instead of two as well as making this variation declarative, and dependent on the operation.

Each terminal unit will be organized in such a way that, there is a master component, and a vector of operation components, those operation components will be categorized in a hierarchical manner. For every node, there will be corresponding 
authentication and authorization attributes, and for every child node, the attributes will be accumulated from the ancestor; the authentication attribute required to unlock a dam, for example, will be an aggregation of the perform, unlock, and dam nodes' authentication attributes. Those attributes will contain the multiplicity $\mathrm{N}$ of the N-Secrecy authentication algorithm, mentioned above, and the authorization workflow required (figure 3.3).

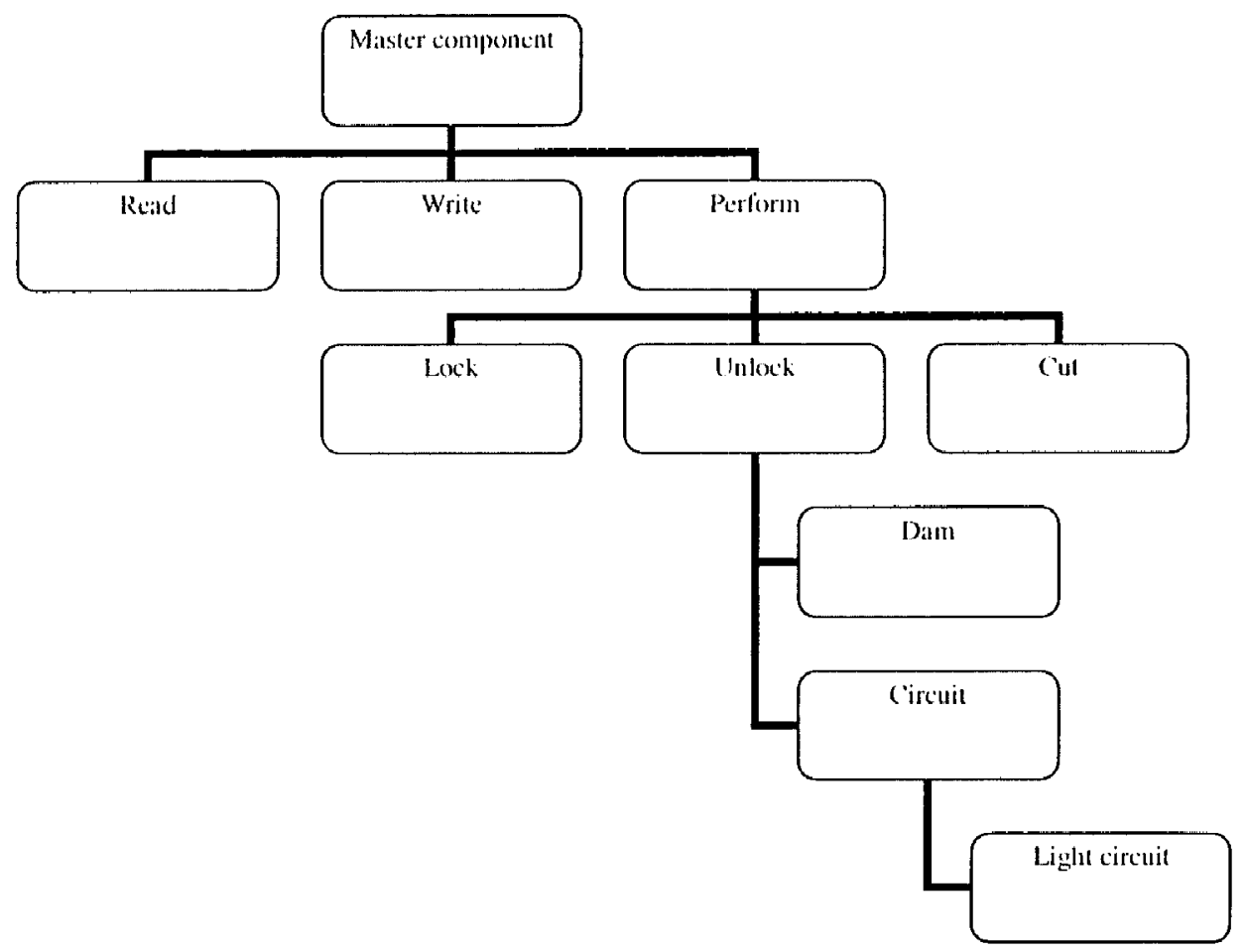

Figure 3.3: Message operation hierarchy example

Hence, the key point here is that there are $n+1$ tests for a message to be authenticated. Now the overall scenario will be that the first secret, which is SSEC0, will contain some information about the type of operation, so that the master component in the terminal unit will forward the message to the right operator component that will have the n-multiplicity to be used so far. The more secrets are read, through the n-Secrecy framework, and testes are passed, the more information the operator component knows about the kind of operation the sender needs, and accordingly forwards to the right node 
beneath it, and then we have a new n-multiplicity. That way the level of authentication is dynamic and depends on the level of importance of some particular operation.

\subsection{Binding the multiple Secrecy algorithms with real SCADA}

This section concerns with the application of the multiple secrecy algorithms with real SCADA systems. In section 2.8, a brief description of the SCADA test bed that is available in the laboratory of the Department of Chemical Engineering was presented. Now, the Double Secrecy algorithm is rewritten below from the perspective of this test bed.

\subsubsection{Double secrecy algorithm using the test bed}

Two wrapper software components will be developed and attached to each party, the MTU and the iFIX PC. The wrapper will work as a façade to the receiver and a mediator to the sender.

The following is the double secrecy algorithm using the above mentioned test bed, see figure 3.4 below,

1. The INPUT.DAT is sent as a message from the sender,

2. The sender Wrapper component gets the message,

3. The header of the message, which can be the time-stamp, contains the secret shared between sender and receiver (SSEC1) encrypted using the GPK,

4. The message body contains the actual message, which is INPUT.DAT, encrypted using the SPK,

5. The footer of the message, which can be the number of characters contained in the INPUT.DAT input file, contains SSEC2, 
6. The message is sent to the receiver, which is the iFIX Wrapper component on the lab PC,

7. The receiver wrapper decrypts the header using the GPK,

8. The receiver wrapper uses its own hash table to look up the message header.

9. If the hash table lookup process results in Null, this would mean an authentication failure. Since, this means that the encrypted script was not SSEC1 in the first place,

10. If the hash-table lookup process succeeds, this will mean that the header is SSEC1, which is then used to get the SPK, and SSEC2,

11. The SPK is then used to decrypt the footer of the message,

12. If the value of the decryption equals the SSEC2, then the message is authenticated,

13. SPK is then used to decrypt the message, and get the INPUT.DAT input file,

14. The input file is then used to run the distillation column process, and the output file OUTPUT.DAT is generated,

15. Now the whole process is repeated to encrypt the OUTPUT.DAT and send it to the MTU and MTU uses the same way to authenticate it at the other side. 


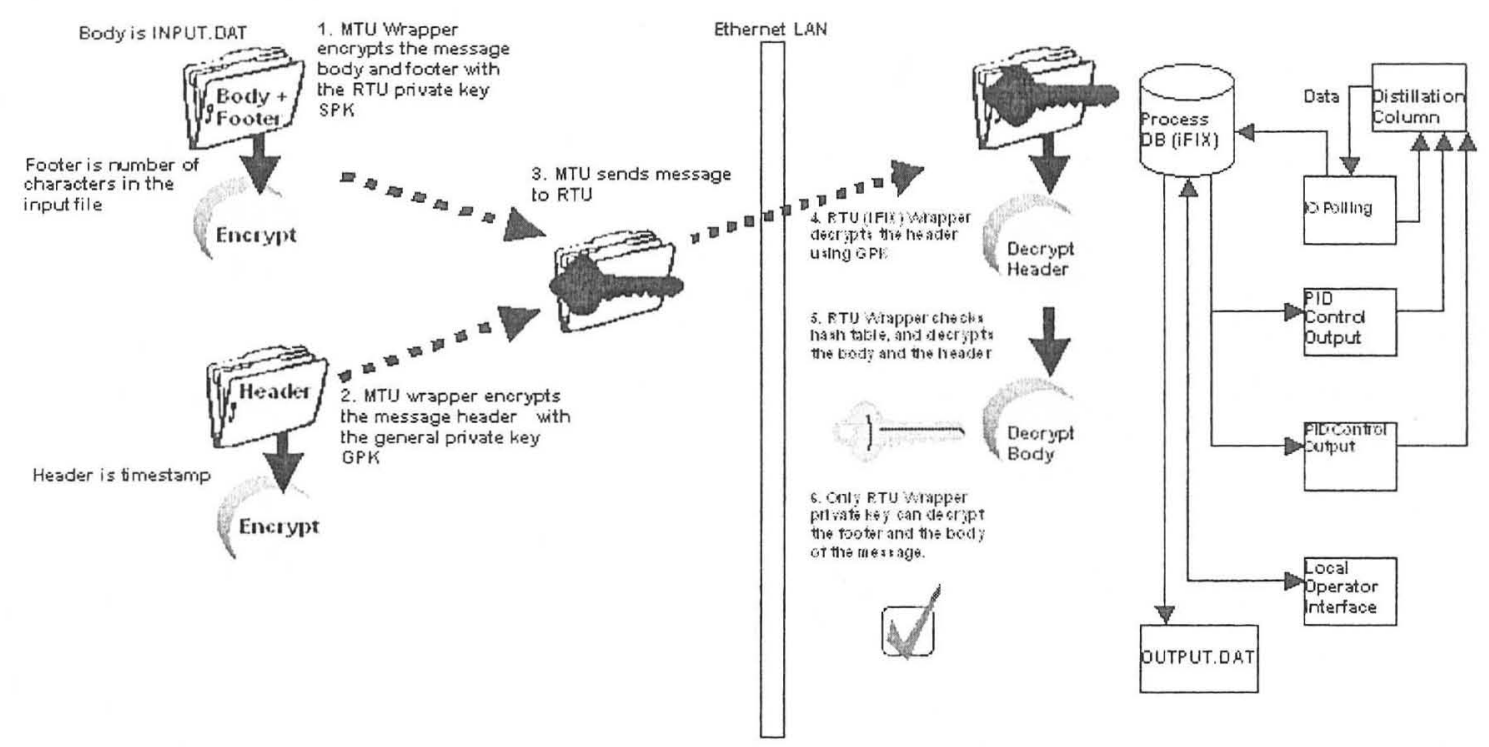

Figure 3.4 Double secrecy framework using distillation column testing bed

\subsection{Multiple Secrecy algorithms and Other Authentication Frameworks}

This section gives brief comparisons between the double secrecy and $\mathrm{N}$-secrecy in general and the other authentication frameworks like SSL, digital signatures, and challenge response.

\subsubsection{Multiple Secrecy and SSL}

The double secrecy depends on two levels of the security, which makes it secure, and the $\mathrm{N}$-secrecy depends on $\mathrm{N}$ secrets, which increases the level of security even more and makes it on demand. From the discussions in 3.2 and 3.4, it is clear that the double secrecy and the $\mathrm{N}$-secrecy in general, do not use public key cryptography, like SSL, because using one-way mappings to encrypt data requires high intensive computation, which would degrade the performance significantly. Hence, the Double and N-secrecy have better performance than the SSL and very secure at the same time.

\subsubsection{Multiple Secrecy and Digital Signatures}


Like the public key encryption, the digital signature framework uses a public key and a private key. In this authentication framework there is a hash digest, which consists of a timestamp and the message to be sent or a part of it. The sender then uses its own private key to encrypt the digest, and sends the message together with the encrypted digest to the receiver. The receiver uses the public key to decrypt the digest, and calculates the digest in its own using the timestamp and the unencrypted received message, and compares the two digests together to decide the authenticity of the sender [33]. Compared with the digital signatures framework, the double and the $\mathrm{N}$-secrecy are more secure because they depend on the validation of at least two secrets and not just one, and the message itself is encrypted, which is not the case in digital signatures, where the plain message is sent unencrypted. The multiple secrecy frameworks are also lighter than the digital signatures framework since it does not use public key encryption.

\subsubsection{Multiple Secrecy and Challenge Response}

The challenge response framework is used when one of the two communicating units need to verify that the other is authentic prior to sending a vital control message or on a periodical manner. In this framework the two units share a secret, and when one of the units try to verify the identity of the other it sends a challenge consisting of a random number. The receiver then adds the secret to the received random number, calculates the hash digest and sends it back to the sender as the response. The sender then compares the challenge and the response to decide the authenticity of the receiver [33]. Comparing the double and the $\mathrm{N}$-secrecy with this framework, they are still more secure because the security depends on more than two secrets and not just one. They are also lighter because the challenge response depends on the digital signature, which in turn depends on the public key encryption. 


\section{CHAPTER IV}

\section{Prototype Implementation and Experimental Results}

In the previous chapter, a new authentication approach was presented. This approach has multiple levels of security, which can vary depending on the level of threat, from Double Secrecy to $\mathrm{N}$-Secrecy. This chapter presents a prototype implementation of both Double Secrecy and N-Secrecy algorithms with the experimental results compared to the SSL results as the opponent authentication framework.

\subsection{Test bed configuration and Testing Methodology}

Testing has been conducted in the Intelligent Systems Research Laboratory (ISRL) at the University of Louisville. Two machines within the SCADA test-bed have been used to simulate the MTU and the RTU. A PC running Windows XP operating system with 2.79 GHz Intel® Pentium ${ }^{\circledR}$ D processor having $2 \mathrm{~GB}$ RAM was used as an MTU and a similar configuration PC was used as the RTU.

Four code packages were implemented, debugged and tested. They are summarized below, with complete listing in Appendix A.

1. A baseline control which is a pure socket connection simulating the DNP3 communication protocol between the two units for a transfer of a simple text file,

2. An implementation of the SSL framework using RSA/IBMx509 and Diffie-Helman as the authentication algorithms,

3. An implementation of the double secrecy algorithm using DES as the encryption algorithm, 
4. An implementation of the double / N-Secrecy algorithms using the Triple-DES as the encryption algorithm.

A component diagram representing the architecture of the prototype is presented in figure 4.1 below. A class called Unit is coded to present the internal authentication functionality of a unit and is then wrapped in two classes, the RTU and the MTU classes, where the DNP3 communication protocol functionality is implemented. The visitor design pattern is used so that according to the parameters passed from the RTU to the MTU or vice versa, the Unit object can use the SSL authentication visitor, the Double Secrecy authentication visitor, the N-Secrecy authentication visitor or no security at all.

Each authentication visitor has its own package where specific implementation classes exist to implement the internal functionality of each type of authentication.

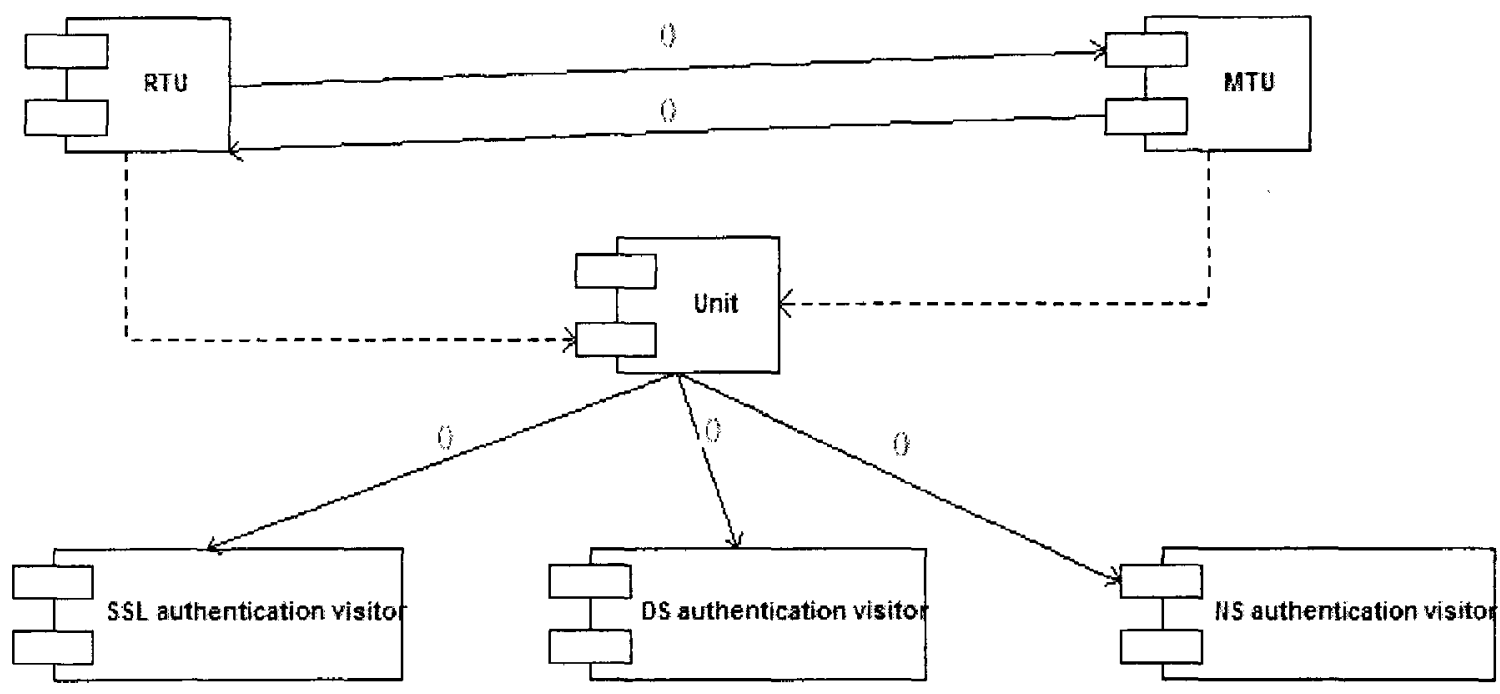

Figure 4.1: Prototype implementation component diagram

\subsection{Double Secrecy and N-Secrecy, Prototype Implementation}

The following sub sections describe the four scenarios of the testing, the one with no security at all, the one with double secrecy, the one with N secrecy, and the one with SSL authentication. 


\subsubsection{No security implementation}

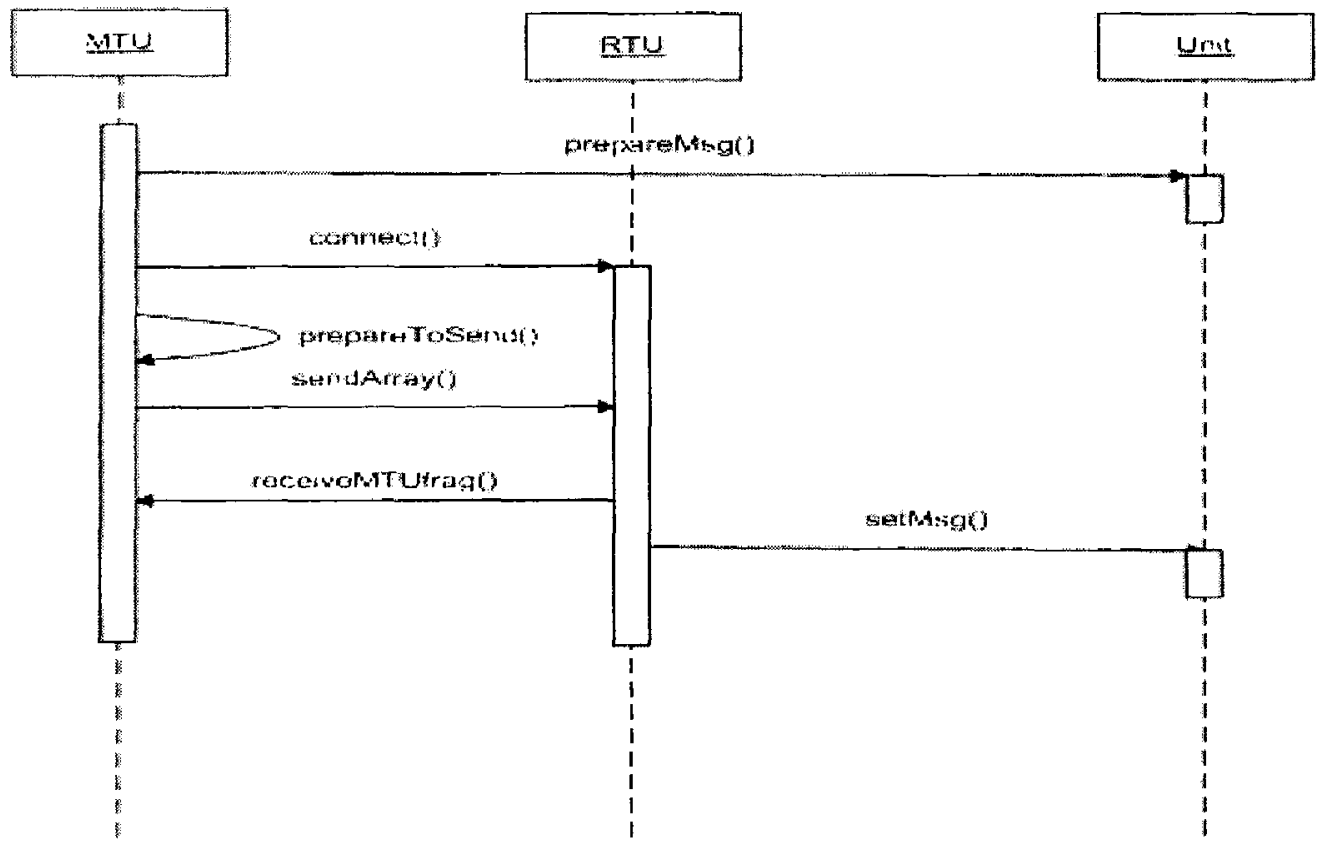

Figure 4.2: No security implementation sequence diagram

Following is the explanation of the no security implementation represented in the sequence diagram above.

1. RTU application class runs first,

2. MTU application class runs, and creates an instance of the Unit class,

3. MTU then calls unit.prepareMsg() which actually does nothing here in the no security package,

4. After the connection happens between the MTU and the RTU, MTU sends the prepared message to the RTU,

5. After, RTU receives the message from the MTU, RTU calls unit.setMsg()

\subsubsection{Double Secrecy implementation}




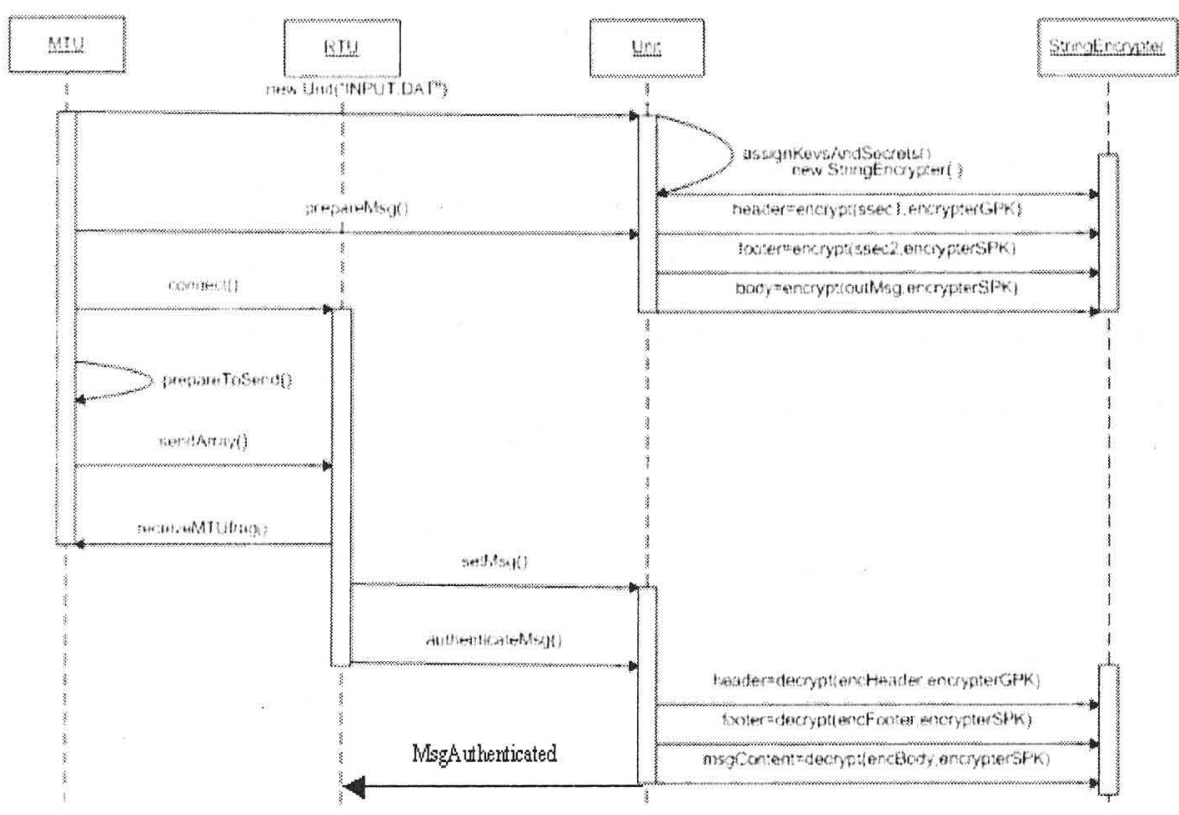

Figure 4.3: Double Secrecy implementation sequence diagram

Following is the explanation of the double secrecy implementation represented in the sequence diagram above.

1. RTU application class runs first,

2. MTU application class runs, and creates an instance of the Unit class,

3. Unit then calls assignKeysAndSecrets() method inside its constructor which creates an instance of the StringEncrypter class,

4. MTU then calls unit.prepareMsg() which in turn calls the encrypt method of Unit which calls the stringEncrypter.encrypt, with the first secret ssec1 and the general key GPK to construct the header of the message,

5. The footer is constructed as the encryption of the second secret ssec2 with the SPK as the encryption key,

6. Finally, the body of the message is constructed as the encryption of the message itself with the SPK as the encryption key, 
7. After the connection between the MTU and the RTU is established, MTU sends the prepared message to the RTU,

8. After, RTU receives the message from the MTU, RTU calls unit.setMsg(),

9. The RTU afterwards calls the unit.authenticateMsg() method to authenticate the message,

10. The Unit instance decrypts the encrypted header with the GPK, using the StringEncrypter decrypt() method, to construct the header of the original message,

11. The footer is is being constructed as the decryption of the encrypted footer with the SPK as the decryption key, and the message content as the decryption of the encrypted body using the SPK as the encryption key.

\subsubsection{N-Secrecy implementation}

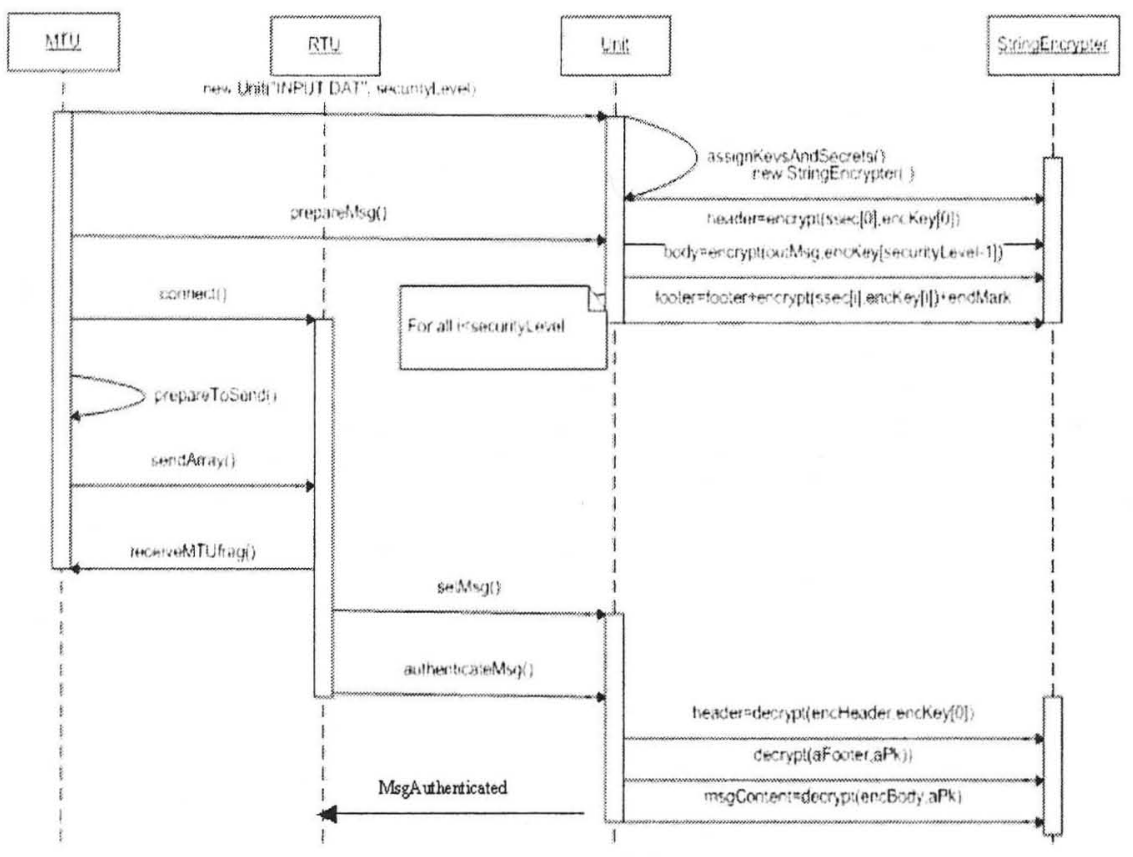

Figure 4.4: N Secrecy implementation sequence diagram

Following is the explanation of the $\mathrm{N}$ secrecy implementation represented in the sequence diagram above.

1. RTU application class runs first, 
2. MTU application class runs, and creates an instance of the Unit class, passing the security level, i.e. the number of secrets required,

3. Unit then calls assignKeysAndSecrets() method inside its constructor which creates an instance of the StringEncrypter class, and sets the hash table containing the pairs (SPKi, SSECi); i=1..n,

4. MTU then calls unit.prepareMsg() which in turn calls the encrypt method of Unit which calls the stringEncrypter.encrypt, with the first secret $\operatorname{ssec}[0]$ and the general key encKey[0] to construct the header of the message,

5. The footer is constructed as the concatenated string composed of the encryption of the secret $\operatorname{ssec}[i]$ using the key encKey[i] for all i< the given securityLevel,

6. Finally, the body of the message is constructed as the encryption of the message itself with the last key in the array encKey[securityLevel-1],

7. After the connection between the MTU and the RTU is established, MTU sends the prepared message to the RTU,

8. After, RTU receives the message from the MTU, RTU calls unit.setMsg(),

9. The RTU afterwards calls the unit.authenticateMsg() method to authenticate the message,

10. The Unit instance decrypts the encrypted header with encKey[0], using the StringEncrypter decrypt() method, to construct the header of the original message,

11. The footer is being constructed inside a loop using the previous step key to decrypt the current step,

12. Finally, the message content is constructed as the decryption of the encrypted body using the final key in the loop.

\subsection{Experimental Results for Double Secrecy}


Experiments were performed to measure the performance between the Double Secrecy, SSL, and the control. A small 4B text file on the MTU machine represents the information to be transferred to the RTU machine. Regular generated triple-DES key sizes; 24B were used as the GPK and SPK in the Double Secrecy implementation.

Secret files ranging from $10 \mathrm{~B}$ to $1 \mathrm{~KB}$ were used for SSEC1 and SSEC2. Results are divided into the following categories: DNP3 messaging with no security implemented; DNP3 with TLS/SSL implemented using Diffie-Hellman as the public key exchange algorithm; and DNP3 with TLS/SSL implemented using RSA as the public key exchange algorithm in table 4-1 and DNP3 with Double Secrecy implemented using DES on a set of secret files and DNP3 with Double Secrecy implemented using Triple-DES on the same set of secret files in table 4-2. Results shown in those tables present the averages of ten different readings. All times were measured in milliseconds.

Table (4.1): Average and standard deviation of DNP3 messaging total time from MTU to RTU with no security implemented, and with SSL implemented

\begin{tabular}{|l|l|l|}
\hline Security framework implemented on DNP3 & Average & Standard deviation \\
\hline No security (control) & 23.1 & 4.77 \\
\hline SSL (Diffie-Hellman) & 2497.6 & 90.5 \\
\hline SSL (RSA) & 2663 & 120.3 \\
\hline
\end{tabular}


Table (4.2): Average and standard deviation of DNP3 messaging total time from MTU to RTU with different combinations of double secrecy

\begin{tabular}{|c|c|c|}
\hline Security framework implemented on DNP3 & Secret file length* & Average \\
\hline \multirow{8}{*}{ Double Secrecy** implemented using DES } & 10 & 50.2 \\
\hline & 20 & 53.4 \\
\hline & 30 & 63.3 \\
\hline & 40 & 59.7 \\
\hline & 50 & 51.5 \\
\hline & 100 & 56.9 \\
\hline & 500 & 550.5 \\
\hline & 1000 & 643.4 \\
\hline \multirow{8}{*}{ Double Secrecy implemented using Triple-DES } & 10 & 45.1 \\
\hline & 20 & 53.7 \\
\hline & 30 & 45.5 \\
\hline & 40 & 73.3 \\
\hline & 50 & 58.3 \\
\hline & 100 & 65.8 \\
\hline & 500 & 573.5 \\
\hline & 1000 & 632.3 \\
\hline
\end{tabular}

*All files' sizes are measured in bytes

**This is the message preparation plus the message authentication time

As shown in the table 4.1 the average of total messaging time in TLS/SSL was 2497.6 milliseconds using Diffie-Hellman as the public key exchange algorithm and 2663 milliseconds using RSA as the public key exchange algorithm, the average of the messaging time in double secrecy, as shown in table 4.2, using DES lies in the average of 50 milliseconds when secrets are shorter than or equal to 100 bytes, and in the average of 500 milliseconds when secrets are larger than or equal to 500 bytes, which is a very large 
size for a secret file. On the other hand, the double secrecy algorithm implemented using Triple-DES encryption showed very similar results, which shows a dramatic improvement of the double secrecy over the TLS/SSL even with large secret files and Triple-DES encryption.

It should be noted that the messaging time mentioned above with regards to the TLS/SSL implementation is only the negotiation/authentication time. There is also the certificates issuance time, which was not investigated here. This is typically a one-time event. However, the major problem with the TLS/SSL is the need for a third party to issue the certificates and the cipher codes. This third party involvement is not a part of the double secrecy algorithm, where everything can be initiated and administered inhouse.

In summary, a simulation of the SCADA systems has been implemented using distributed machines switching messages over the DNP3 protocol. The messaging has been done using no security at all, using TLS, and using the double secrecy for authentication on small and larger secrets over using the DES and Triple-DES as the encryption algorithms. The results showed that Double Secrecy using DES or Triple-DES gives a response time within the service level limits of almost all process system SCADA installations, and that this response time is significantly better than that using the TLS/SSL as a method for authentication.

\subsection{N-Secrecy, Experimental Results}

Results of N-Secrecy implemented using Triple-DES on large secrets (100bytes) are shown in Table 4.3, using three, four, and five secrets respectively. In those results ten 
readings were taken and the averages and standard deviations were computed. All times were measured in milliseconds.

Table (4.3): Average and standard deviation of DNP3 messaging total time from MTU to RTU with $\mathrm{N}$-Secrecy implemented using Triple-DES on large secret files using $\mathrm{N}=3,4,5$, and 6

\begin{tabular}{|l|l|}
\hline Number of secrets used for N Secrecy & Average \\
\hline $\mathrm{N}=3$ & 530.5 \\
\hline $\mathrm{N}=4$ & 760.2 \\
\hline $\mathrm{N}=5$ & 945.6 \\
\hline $\mathrm{N}=6$ & 1020.4 \\
\hline
\end{tabular}

As shown in table 4.3 the average of total messaging time in $\mathrm{N}$-secrecy using TripleDES with large three secrets was 530.5 milliseconds, the average using four large secrets was 760.2 milliseconds, the average using five large secrets was 945.6 milliseconds, and finally the average using six large secrets was 1020.4 which still shows a dramatic improvement over the TLS/SSL even with large secret files and Triple-DES encryption, and multiple secrets corresponding to the state of the attack.

It should also be noted that this total time is the message preparation time plus the message authentication time. Since SCADA operations are known upfront for both units, the total time can be further improved by preparing a large portion of the message at the start up of both units.

In summary, a simulation of the SCADA systems has been implemented using distributed machines exchanging messages over DNP3 protocol. The messaging has been done using $\mathrm{N}$-secrecy with three, four and five large secrets, over triple DES encryption and compared with the results obtained from the TLS. Results showed that increasing the number of secrets did not affect the total messaging time. 


\section{CHAPTER V}

\section{Computer Algebra techniques for Enhancing SCADA security}

\subsection{Introduction}

When two parties, typically two units or an RTU with MTU, try to authenticate each other prior to sending any vital information, or committing any vital transaction at the other side, they must share a secret. In a computation, this secret is a string of binary characters. The power of the secret/key combination, and how difficult it is to break, is the most important measure of the power of the security system implemented in the communication between those two parties. The problem is how to share the secret information. Should it be done through a secured line of communication, but how can this communication line be judged to be secure enough, and how can a successful authentication process be conducted on it?

This problem of key exchange has two common solutions: 1.) the Diffie-Hellman algorithm that depends on the difficulty of computing the discrete logarithm problem and is equivalent to the integer factorization problem, and 2.) the RSA algorithm that depends on the difficulty of factoring large prime numbers. Recently, elliptic curve public cryptography was developed [32]. All of these commonly used algorithms depend on the complexity of some problems in the theory of numbers. In this chapter, new algorithms are presented to model the real setting of the two communicating units, and the communication line in between, using polynomial ring theory with the Grobner bases algorithm to complete the solution. 
First, the Security Spectrum algorithm is discussed, and then a detailed example is presented. A comparison between this algorithm and Diffie-Helman algorithm concludes the chapter.

\subsection{Solution overview}

The cornerstone in the solution is to build a logical bridge between the two parties. This bridge will be referred to as the security spectrum of the two parties. The security spectrum between any two parties is unique to these parties. It changes if any one of the intrinsic or boundary conditions of either one of the two parties or the line of communication between them changes. Each one of the two parties generates its secrets individually and on a periodical manner. The way the authentication happens is by checking the correlation between the secret and the spectrum. This means that the two secrets generated on the two parties need not be the same but they should be correlated to the same spectrum. There will be a level for this correlation, so that each piece of transaction will have a threshold for the level of correlation accepted.

\subsubsection{Issues}

There are several issues that must be addressed. A first key issue is to find a way to model the intrinsic, boundary, and communication conditions for the two communicating parties, to build up the spectrum, then find a way for party A to generate a random secret correlated with that spectrum. The user must find a computationally reasonable way to encode that secret. In operation, the user must decode the secret at party B in a one-one corresponding encoding/decoding fashion, and finally make party B decide for certain if the secret is correlated to the spectrum between that party, and party A, that is to find a unique solution for the decidability problem. 


\subsubsection{Solutions}

First addressing the conditions representation, each condition can be thought of as a non linear function of some physical variables, model these physical variables as symbols, and model the whole condition as a non-linear polynomial of these symbols, which will then be the variables of that polynomial. Then the whole condition can be represented geometrically as the variety object in the space $\mathrm{K}^{\mathrm{m}}$ where $\mathrm{K}$ is the field in which the ground values of the variables belong to, typically the field of real numbers, and $m$ is the space dimension. Modeling all the conditions that way, as a system of nonlinear polynomials, corresponding to a set of varieties in the space $\mathrm{K}^{\mathrm{m}}$ makes the area of intersection of these varieties resembles the ideal generated by all these polynomials. That way we can generate our spectrum as the ideal generated by the system of nonlinear polynomials representing the physical conditions of communication between the two parties. (An overview of ideals is included in Appendix B).

Now addressing the issue of the correlation, this can be found in the answer of the question "does this secret, represented as a new polynomial, rely in the ideal generated by the conditions' polynomials or not?" To generate a new secret at either party that is correlated to the spectrum, a polynomial that is a linear combination of the conditions' polynomials, and clearly there are an infinite number of these combinations, will be correlated to the spectrum for sure. Finally, for the other party to decide if the sent polynomial, representing the secret, is authenticated or not, that is to say if it is correlated to the spectrum or not, it is the same as to say that the polynomial belongs to the ideal generated by the spectrum of conditions' polynomials. 
This is equivalent geometrically to decide if some geometrical variety belongs to the intersection area between a group of varieties or not. This previously mentioned problem is called the uniform word problem, and the problem is that the solution to this problem is not unique. Therefore another approach is needed, and for this research the Grobner Basis is used to resolve the non-uniqueness problem.

\subsection{Security Spectrum Algorithm}

Before stating the security spectrum algorithm, it is worth noting that this algorithm, like all the key distribution algorithms, runs as an offline operation. Following is the detailed algorithm for the Security Spectrum approach:

1. Intrinsic conditions of each of the two parties are gathered,

2. Physical conditions of the communication between the two parties are gathered,

3. A modeling of all these conditions is done to generate a group of polynomials describing the whole system of communication,

4. The security spectrum of the two parties is constructed as the Grobner basis of this system of polynomials,

5. Party A generates a linear combination of the polynomials constructing the spectrum, to be the secret, this secret

6. The secret gets encoded, and sent to party B,

7. Party B decodes the secret,

8. Party $\mathrm{B}$, runs the uniform word problem algorithm, to decide uniquely if the secret sent is correlated with the spectrum or not, which is an answer to the authentication problem, 
9. Party B, runs the correlation level algorithm to determine the level of correlation, and decide if this level of correlation authorizes party $\mathrm{A}$, or not. An answer to the authorization problem.

\subsection{Using the Security Spectrum algorithm in a SCADA application}

As done in chapter III, this section concerns with the application of the Security Spectrum algorithm with real SCADA systems. The simplified version of the test bed available using the Chemical Engineering Department distillation column is considered as an emulator for a real SCADA systems.

First the intrinsic conditions of each of the MTU and the iFIX, basically all the control loops and all the relevant equations are gathered. For these controls case, these will be the processing blocks, which will generate a system of polynomials describing the whole system of communication. Next the security spectrum of the two parties is constructed using the Grobner basis of this system of polynomials. Afterwards, party A, MTU wrapper generates a linear combination of the polynomials constructing the spectrum, to be the header of the message, the INPUT.DAT input file is encoded using this header and put as the body of the message. The message then gets sent to party B, the iFIX. On the other side, party B wrapper, runs the uniform word problem algorithm, to decide uniquely if the header of the sent message, the secret, is correlated with the spectrum or not, an answer to the authentication problem. Party B wrapper, runs the correlation level algorithm to determine the level of correlation, and decide if this level of correlation authorizes party A, or not, an answer to the authorization problem. If the message is authenticated the body is decrypted using the header, and the input file is then used to run the distillation column process, and the output file OUTPUT.DAT is 
generated. Now the whole process is repeated to encrypt the OUTPUT.DAT and send it to the MTU. MTU will use the same way to authenticate it at the other side. This is all shown in a block diagram form in Figure 5.1.

\subsection{Example}

The following simplified example uses a control model system to demonstrate the approach. Let's take as an example, one of the distillation column control blocks called PID block, which is intended to maintain balance in a closed loop by changing the controlled analog input variable in response to deviations from a user defined set point.

\subsubsection{Collecting the intrinsic and boundary conditions}

The PID block itself has two typical flows, the PID block's feedback tag illustrated below in Figure 5.2, and a specific cascade control with Master/Slave PID blocks Figure 5.3. In addition we have the steady state PID block algorithm equation.

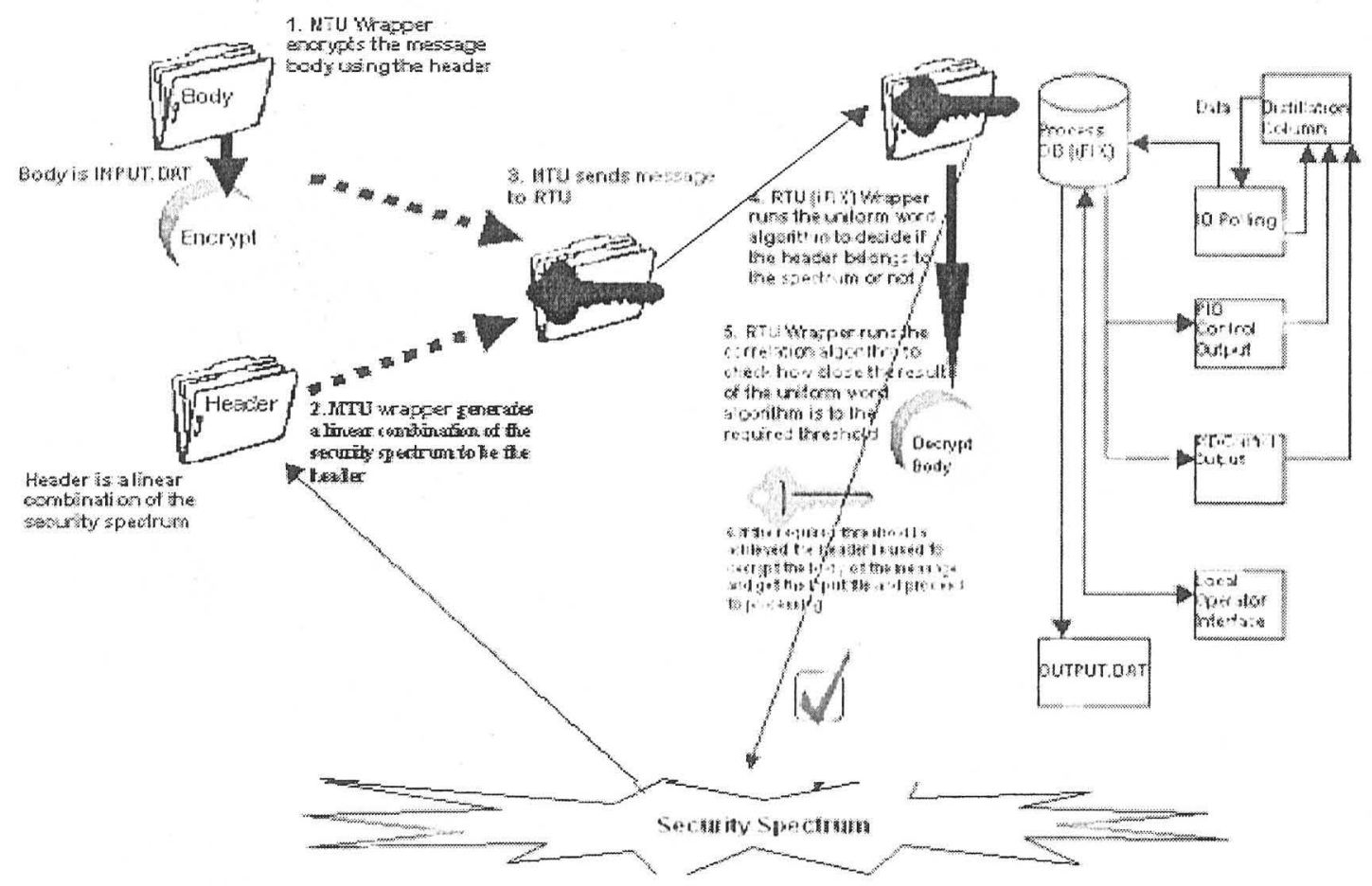

Figure 5.1 Security Spectrum framework using distillation column testing bed 


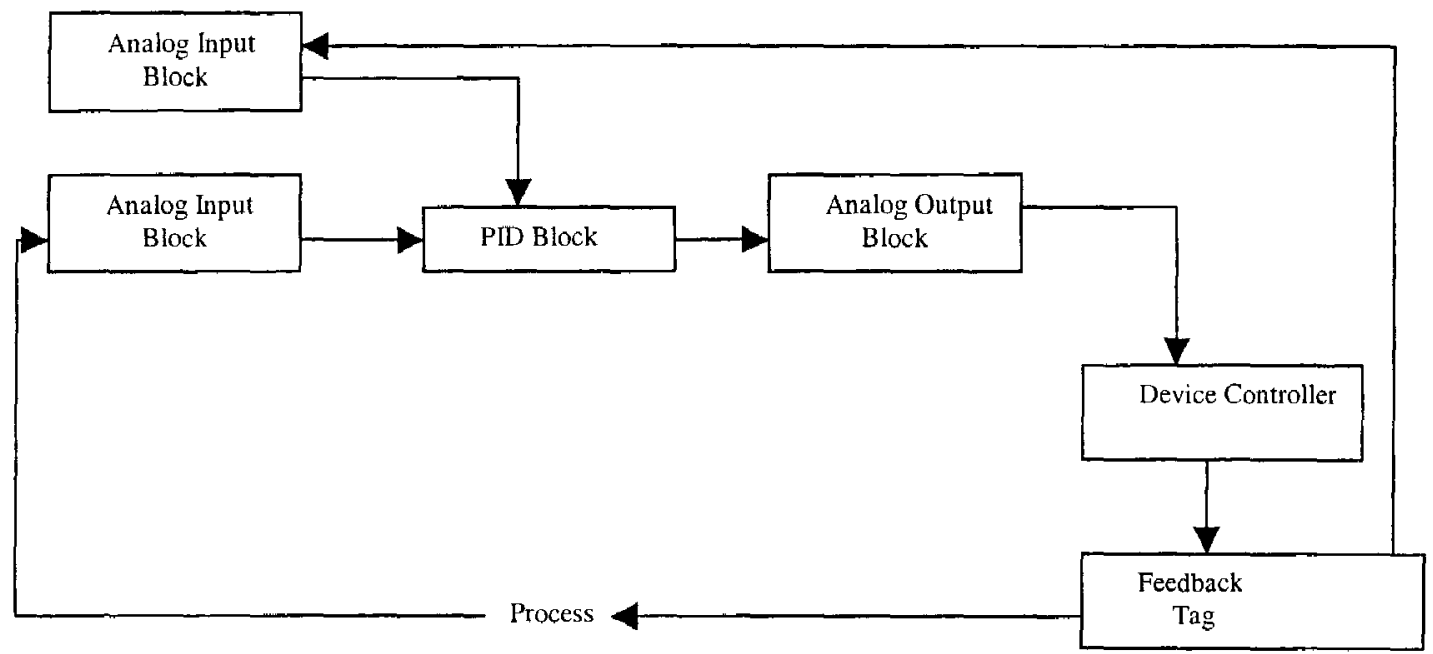

Figure 5.2 PID block's feedback tag

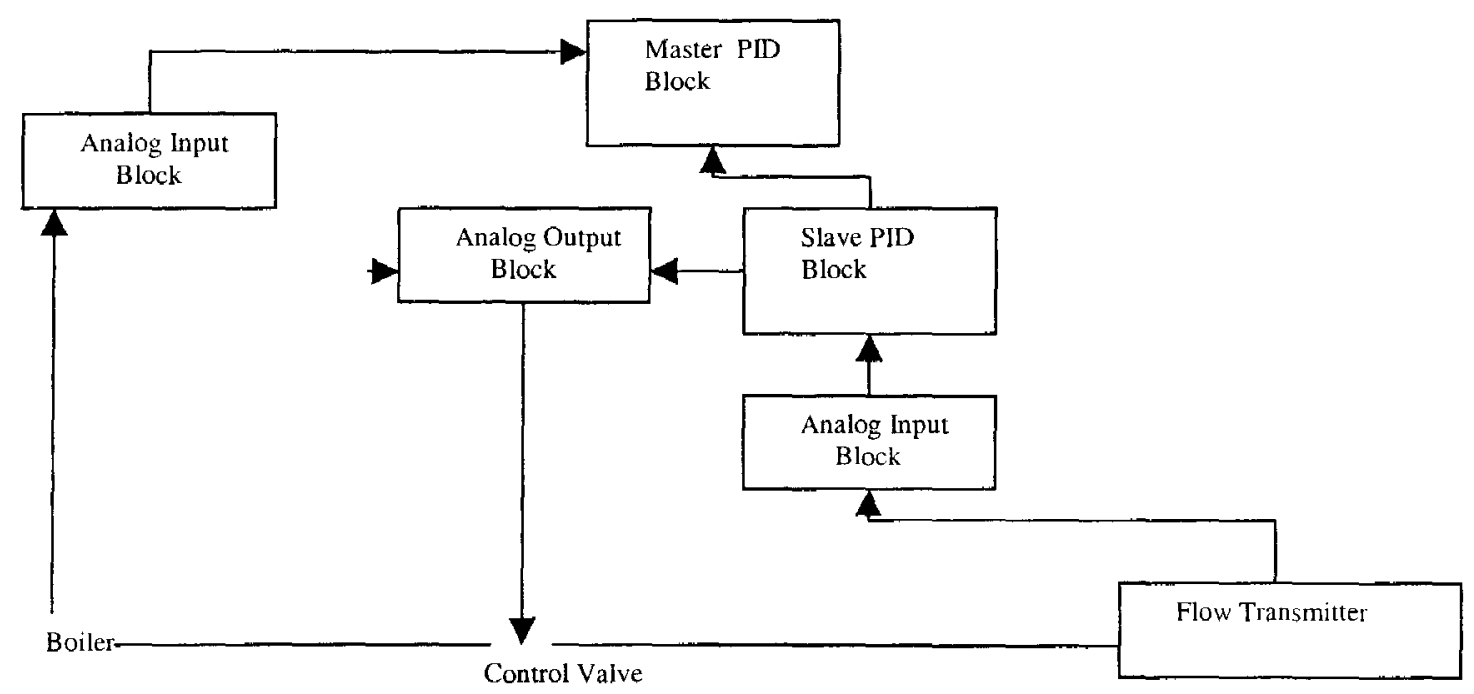

Figure 5.3 cascade control with Master/Slave PID blocks

\subsubsection{Modeling the conditions in polynomial format}

Here are the Representation rules,

1. Each block is a symbol,

a. $\mathrm{x}_{0}$ for PID block,

b. $x_{1}$ for Analog Input block,

c. $x_{2}$ for Analog Output block, 

d. $x_{3}$ for Feedback tag,
e. $x_{4}$ for Analog Input block II,
f. $x_{5}$ for Slave PID block,
g. $\mathrm{x}_{6}$ for Master PID block,
h. $x_{7}$ for Flow transmitter,
i. $\mathrm{x}_{8}$ for the control valve,
j. $\mathrm{x}_{9}$ for the boiler.

2. The flow from one node $X$ to another $Y$ is represented by the monomial $X Y$,

3. The flows from node $\mathrm{X}$ to another $\mathrm{Y}$, and then from node $\mathrm{Z}$ to $\mathrm{C}$, is given by the polynomial XY $+\mathrm{ZC}$.

Following the above-mentioned rules, we have the following polynomials,

For Fig5.2,

1. $\mathrm{P}_{1}=\mathrm{x}_{1} \mathrm{x}_{0}+\mathrm{x}_{0} \mathrm{x}_{2}+\mathrm{x}_{2} \mathrm{x}_{3}+\mathrm{x}_{3} \mathrm{x}_{1}$,

2. $P_{2}=x_{1} x_{0}+x_{0} x_{2}+x_{2} x_{3}+x_{3} x_{4}+x_{4} x_{0}$,

For Fig5.3,

1. $\mathrm{P}_{3}=\mathrm{x}_{7} \mathrm{x}_{1}+\mathrm{x}_{1} \mathrm{x}_{5}+\mathrm{x}_{5} \mathrm{x}_{2}+\mathrm{x}_{2} \mathrm{x}_{8}+\mathrm{x}_{9} \mathrm{x}_{4}+\mathrm{x}_{4} \mathrm{x}_{6}$,

2. $\mathrm{P}_{4}=\mathrm{x}_{7} \mathrm{x}_{1}+\mathrm{x}_{1} \mathrm{x}_{5}+\mathrm{x}_{5} \mathrm{x}_{6}+\mathrm{x}_{5} \mathrm{x}_{2}+\mathrm{x}_{2} \mathrm{x}_{8}+\mathrm{x}_{9} \mathrm{x}_{4}+\mathrm{x}_{4} \mathrm{x}_{6}$,

Buchberger's algorithm for computing Grobner Bases was implemented, see appendix A for code listings. The idea behind Buchberger's algorithm is simply to order the polynomials of the whole system using a given term order, and then construct a type of polynomials designated as the s-polynomials. An s-polynomial is constructed from two polynomials by removing the leading terms of both of them using the least common multiple term for their leading terms, and then subtracting one from the other. 
Afterwards, the resultant polynomial is checked for the existence in the ideal by running the Euclidean division algorithm. If the s-polynomial is not in the ideal, it is added to the system of polynomials and another couple of polynomials is chosen to compute the new s-polynomial, and so forth until all the polynomials in the system prove to be in the ideal.

\subsubsection{Algorithm Snapshot}

Having modeled the control blocks in polynomials format, below are the full steps of the algorithm.

1. The polynomials should be ordered in the right term order in order to compute Grobner basis for them. Assuming that $x_{0}>x_{1}>x_{2}>\ldots x_{n}$ and so forth, the original system of polynomials is:

$$
\begin{aligned}
& P_{1}=x_{0} x_{1}+x_{0} x_{2}+x_{1} x_{3}+x_{2} x_{3}, \\
& P_{2}=x_{0} x_{1}+x_{0} x_{2}+x_{0} x_{4}+x_{2} x_{3}+x_{3} x_{4}, \\
& P_{3}=x_{1} x_{5}+x_{1} x_{7}+x_{2} x_{5}+x_{2} x_{8}+x_{4} x_{6}+x_{4} x_{9}, \\
& P_{4}=x_{1} x_{5}+x_{1} x_{7}+x_{2} x_{5}+x_{2} x_{8}+x_{4} x_{6}+x_{4} x_{9}+x_{5} x_{6}
\end{aligned}
$$

2. Running the Grobner bases code, the following system of polynomials is obtained,

$$
\begin{aligned}
& P_{1}=x_{0} x_{1}+x_{0} x_{2}+x_{1} x_{3}+x_{2} x_{3}, \\
& P_{2}=x_{0} x_{1}+x_{0} x_{2}+x_{0} x_{4}+x_{2} x_{3}+x_{3} x_{4}, \\
& P_{3}=x_{1} x_{5}+x_{1} x_{7}+x_{2} x_{5}+x_{2} x_{8}+x_{4} x_{6}+x_{4} x_{9}, \\
& P_{4}=x_{1} x_{5}+x_{1} x_{7}+x_{2} x_{5}+x_{2} x_{8}+x_{4} x_{6}+x_{4} x_{9}+x_{5} x_{6}, \\
& P_{5}=-x_{0} x_{4}+x_{1} x_{3}-x_{3} x_{4} \\
& P_{6}=-x_{0}{ }^{2} x_{2}-x_{0}{ }^{2} x_{4}-x_{0} x_{2} x_{3}+x_{0} x_{2} x_{5}-x_{0} x_{3} x_{4}+x_{1} x_{3} x_{5}+x_{2} x_{3} x_{5}, \\
& P_{7}=-x_{0} x_{1} x_{7}-x_{0} x_{2} x_{8}-x_{0} x_{4} x_{6}-x_{0} x_{4} x_{9}-x_{0} x_{5} x_{6}+x_{1} x_{3} x_{5}+x_{2} x_{3} x_{5},
\end{aligned}
$$




$$
\begin{aligned}
& P_{8}=-x_{0} x_{1} x_{7}-x_{0} x_{2} x_{8}-x_{0} x_{4} x_{9}+x_{2} x_{3} x_{5}+x_{3} x_{4} x_{5} \\
& P_{9}=-x_{0} x_{1} x_{7}-x_{0} x_{2} x_{8}+x_{0} x_{4} x_{5}-x_{0} x_{4} x_{6}-x_{0} x_{4} x_{9}-x_{0} x_{5} x_{6}+x_{3} x_{4} x_{5} \\
& P_{10}=-x_{5} x_{6}
\end{aligned}
$$

3. Now assume that the MTU needs to send the transaction 'abc' to the RTU. The MTU will construct a message composed of a message header and a body, where the body of the message will be the transaction content,

4. The header of the message will be the secret. This secret will be any linear combination of the ten polynomials above representing the ideal, or the security spectrum,

5. Let the header be: $10 \mathrm{P}_{5}-1.5 \mathrm{P}_{10}+\mathrm{P}_{8}=$

$$
-x_{0} x_{1} x_{7}-x_{0} x_{2} x_{8}-x_{0} x_{4}\left(10+x_{9}\right)+10 x_{1} x_{3}+x_{2} x_{3} x_{5}-x_{3} x_{4}\left(10+x_{5}\right)-1.5 x_{5} x_{6}
$$

6. The message is sent to the RTU,

7. RTU runs the uniform word problem code, see Appendix A, to resolve the decidability of the existence of the header in the spectrum,

8. The uniform word problem code gives an affirmative response and the MTU is authenticated,

9. The message body is extracted.

\subsubsection{Analysis of the security spectrum algorithm example snapshot}

Noting how large the above system of hypothetical non-linear polynomials is, and knowing the fact that in reality we may have hundreds of those non-linear polynomials modeled using more sophisticated models, it is extremely unlikely that an intruder can spoof the RTU or the receiver in general. So the confidence that no unauthorized person or machine can send a false message is very high. 
The real problem is with the man-in-middle attack since an intruder cutting in the middle and getting the message will simply get the message in the message body, which can be an important information. The solution to this problem is to use the security spectrum only to generate the secret(s) and use the double secrecy or the Nsecrecy in general to do the whole authentication framework. In other words, use the $\mathrm{N}$-secrecy as the authentication framework and the security spectrum as the secret generator and authorization framework. In this case the security spectrum algorithm can be run on an offline basis to generate the secrets and the keys needed in the Nsecrecy and transfer those keys and secrets via ROM device into the SCADA units as part of the administration of the system, and then those keys and secrets can be used on an online basis to secure the transactions. Multiple channels can also be used to resolve the key distribution problem, by generating the keys and secrets and emailing them to the other administrators of the distributed SCADA system, and then using the $\mathrm{N}$-secrecy authentication framework. All the steps of the above example use the security spectrum as the key generator algorithm and the double secrecy as the authentication framework, as shown in Figure 5.4.

\subsection{Security Spectrum and Diffie-Hellman}

The whole difference between the security spectrum and Diffie-Hellman algorithms is the fact that the latter depends on the theory of numbers, the modulo ring theorem and the complexity of the discrete logarithm problem as mentioned in section 1.1.2, whereas, the security spectrum depends on the real context of the problem of communication between the two parties, and the complexity of resolving the uniform word problem as mentioned in appendix B. Diffie-Hellman, is also known to be 
vulnerable to the man in middle attack, because an intruder can simply intercept a party's public value and sends a different one to the other party. That way, the intruder can decrypt all the messages sent between the two parties and even more, can send his own fake messages. An intruder intercepting any messages from two communicating parties over the security spectrum framework, on the other hand, will not benefit at all from that piece of information neither by understanding nor by using it to later deceive one of the two parties because it depends on the context of the problem not on a known mathematical problem.

A further note is that the security spectrum can strengthen by wrapping the whole framework by the N-Secrecy framework. That is to use the N-Secrecy to encode/decode the secret polynomial that is being sent between the two parties. 

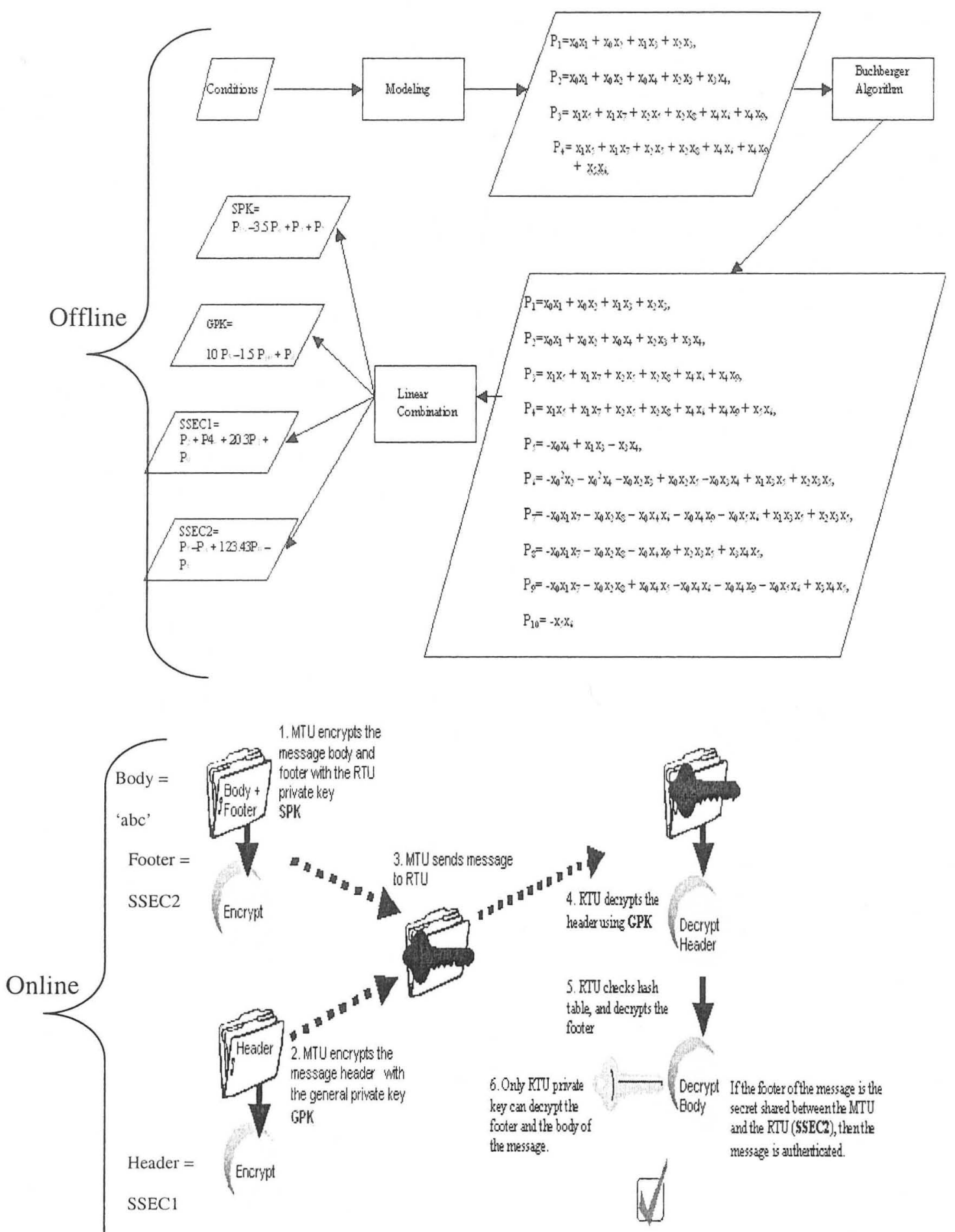

Figure 5.4 Using security spectrum as the key generator algorithm and double secrecy as the authentication framework 


\section{CHAPTER VI}

\section{Conclusions and Future Directions}

In the introduction of this dissertation an argument was presented on whether it is better to use well known security technologies and frameworks like the SSL/TLS or to use tailored frameworks to secure the SCADA systems.

The argument was that the well-known frameworks are more mature and less expensive but on the other hand they can cost a lot on the performance side. Hence, the objective of this dissertation is to find new cryptographic approaches that have the same security level offered by those well-known security frameworks, particularly the SSL, without compromising the performance. Two trials were done in this dissertation to achieve the above-mentioned goal, named the N-Secrecy and the security spectrum. The concluding remarks regarding the two frameworks are presented below.

\subsection{Conclusions}

\subsubsection{N-Secrecy}

In chapter three the idea of the multiple levels of secrecy was first introduced. The double secrecy algorithm was presented and clearly it depends on symmetric (private key) encryption using two secrets and two keys to encrypt and decrypt those secrets, which means that there is no use at all for the trapdoor one way mapping that the public key encryption algorithms like the RSA, ECC, and Diffie-Helman depend upon. This means that the double secrecy should be better than the SSL in terms of performance. As for the security, later sections in the same chapter discussed the strength of the double 
secrecy against the Spoofing-DOS attack, where an inspector component was added to the framework to take the first packet of the message header and do some pattern matching to check if it is a promising packet to be a part of some key in the hash-table or not. Afterwards, the level of secrecy was extended from two to a general number $\mathrm{N}$ depending on the level of secrecy desired based maybe on the level of confidentiality or vitality the message has.

Chapter IV presented the experimental results of the double secrecy using a number of secrets ranging from 10bytes to one KB. Results showed that the double secrecy took in the range of one thousandth of the SSL running time in most of the secrets used. Also, the $\mathrm{N}$-secrecy experimental results represented in the same chapter showed that using a large secret files and Triple-DES as the symmetric encryption algorithm still cost much less than the SSL even when the level of secrets was increased to 6 .

\subsubsection{Security Spectrum}

Chapter V presented another new security framework called the security spectrum which tried to construct a physically meaningful bridge between the two communicating parties rather than depending on meaningless complex mathematical problems like the problem of discrete logarithm in the case of Diffie-Helman and the non inverse mapping prime numbers problem in case of the RSA. The complexity of the problem here depends on building a logical bridge composed of polynomials representing physical conditions of the two communicating parties and/or the communicating network.

An example was represented in the chapter to present the whole idea. The example however, used a trivial way of modeling the conditions into polynomials. Next 
section in this chapter presents a novel mathematical modeling technique for control systems. Work on this modeling technique can be completed in a future research work.

\subsection{Directions for Future Research}

\subsubsection{Modeling}

This section focuses on presenting a novel mathematical model for control systems using context free grammars and non-deterministic finite automata.

A typical control system consists of flows of control blocks, and flows from control blocks to each others. These flows can happen in sequence, or they can happen in parallel. A typical control system also contains some processing, and valves for multiple branches.

\subsubsection{Modeling using context free grammars}

A map from a control system to a grammar, can be built such that, a non trivial block is considered a non terminal block, each final operation is considered a terminal block, each flow from a block to another is considered a production rule, and finally, each valve is considered an "or" operation in the corresponding production rule.

Using this map, a control system can be considered a federation of many grammars generating a language of transactions based on the different production rules relevant to the different flow control blocks. Each branch on the syntax tree generated by a given grammar can be modeled as a monomial of the block terms. Summing up all the branches using the " + " operator, a polynomial representing this block can be generated for that given grammar for that given block. A system of polynomials can then be generated for all the blocks in the system. 
In the next two sections the ground group and ring will be constructed based on the syntax tree representing the control system grammar transactions. Once this ground ring has been constructed the corresponding polynomial ring can then be used to build up the model.

\subsubsection{Modeling using context free grammars, Group and Ring construction}

To sum up, given a forest of transactions trees like the one in figure 6.1 below, we have the following,

- Consider the set of all symbols representing the nodes in the forest,

- The direct flow from a node $\mathrm{X}$ to a node $\mathrm{Y}$ is represented as a monomial $\mathrm{XY}$,

- The different branches are to be represented as an addition, like $\mathrm{XY}+\mathrm{XZ}$,

- The feedback from a child node to a parent node depending on a condition and the number of iterations is to be represented as a non-linear monomial multiplied by the condition.

Considering all the above mentioned points that set of symbol nodes can be considered a ring under the ordinary addition and multiplication of the ring of real numbers.
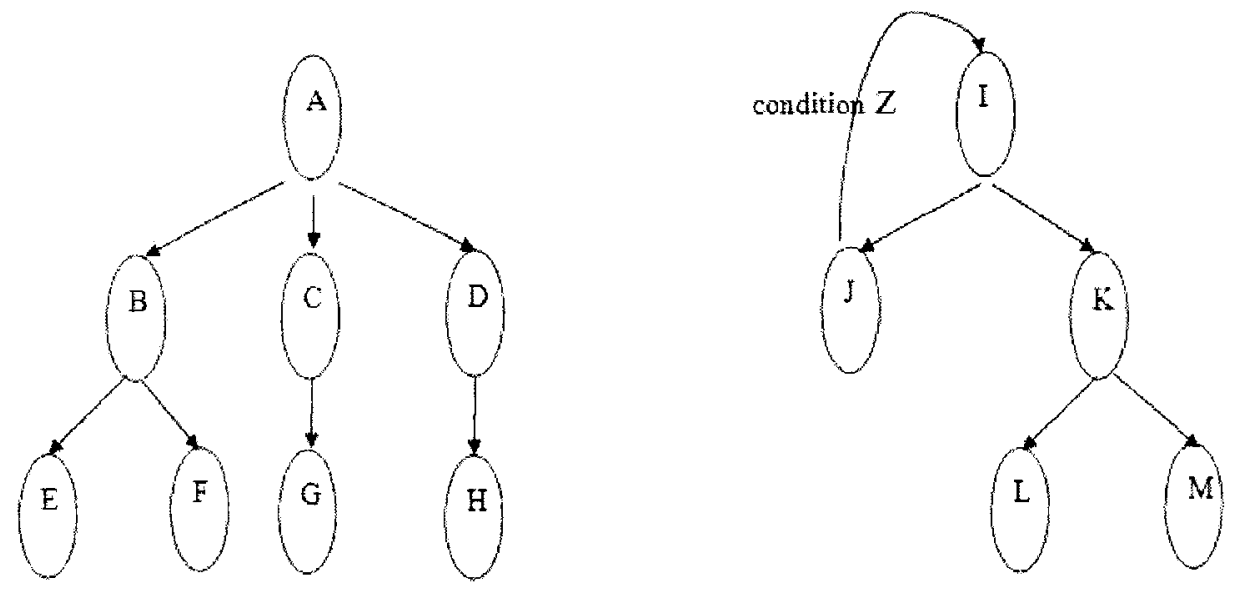

Figure 6.1 Transactions forest 
From figure 6.1 above the following system of non-linear polynomials can be modeled:

$$
\begin{aligned}
& \mathrm{ABE}+\mathrm{ABF}+\mathrm{ACG}+\mathrm{ADH} \\
& \mathrm{ZI}^{2} \mathrm{~J}^{2}+\mathrm{IKL}+\mathrm{IKM}
\end{aligned}
$$

assuming we can only have two repetitions in that path. Now the rest of the algorithm can be completed the normal way by getting Grobner basis for the above system of non-linear polynomials.

\subsubsection{Computations complexity enhancement}

Grobner bases and uniform word problem algorithms are essential parts of the security spectrum algorithm and should perform in an acceptable manner in order for the whole framework to work in the proper service level. The algorithms used for both of those problems are the classic Buchberger and uniform word decidability problem algorithms, which are both NP-complete [34]. Future researchers should try to enhance those two algorithms, or they may be able to find another way that is not as constructive as the classic algorithms, but performs better.

\subsubsection{Testing}

All the results presented in the dissertation obtained from emulating the SCADA and DNP3 as described in section 4.1. One of the strongest candidates for future research is to test the approaches presented in this dissertation in real SCADA systems such as the University of Louisville test bed. 


\section{REFERENCES}

[1] US Department of Energy, Importance of Energy Control Systems to Protecting Critical National Infrastructures, http://www.oe.energy.gov/439.htm, accessed October 2007.

[2] Committee on Science and Technology for Countering Terrorism, National Research Council, Making the Nation Safer: The Role of Science and Technology in Countering Terrorism. http://www.nap.edu/catalog/10415.html, accessed May 2007.

[3] U.S. Department of Energy, (2006). The Role of Authenticated Communications for Electric Power Distribution Pacific Northwest National Laboratory, accessed November 2007.

[4]NERC Control Systems Security Working Group, http://www.nerc.com/ filez/csswg.html, accessed Sept 2007.

[5] National Science Board, National Science Foundation, (2002). Science and Engineering Indicators, Volume 2, NSB-02-2, Arlington, http://www.nsf.gov/sbe/srs/, accessed November 2007.

[6] Knezo, G. J., (2002). Possible Impacts of Major Counter Terrorism Security Actions on Research, Development, and Higher Education, Congressional Research Service, http://www.fas.org/irp/crs/RL31354.pdf, accessed December 2007.

[7] SEL-3332 Intelligent Server, www.selindustrial.com, accessed Sept 2007. 
[8] Xavier, P., and Lluis, M (2006). An introduction to SCADA systems security, accesses January 2008.

[9] Venkat, P.., and Matthew, F., (2006). SCADA HoneyNet Project: Building Honeypots for Industrial Networks, Critical Infrastructure Assurance Group(CIAG), Cisco Systems, Inc, accessed January 2008.

[10] SCADA Safe project documentation, http://scadasafe.sourceforge.net, accessed October 2007.

[11] IT Security Expert Advisory Group, http://www.tisn.gov.au, (2005). Supervisory Control and Data Acquisition SSCADA Security-Advice for CEOs, accessed December 2007.

[12] Roadmap to Secure Control Systems in the Energy Sector, http://www.oe.energy.gov/randd/438.htm, accessed November 2007.

[13] http://en.wikipedia.org/wiki/Technology Readiness Level, accessed September 2007.

[14] Control Systems Security, Project Summaries, Arlington, Virginia, (2006), accessed April 2007.

[15] Donald, I., W., (2003). Internet-based SCADA Comes of Age. M2M Data Corp.

[16] National Institute of Standards and Technology, (2006). Guide to SCADA and Industrial control systems security, SP 800-82, accessed January 2007.

[17] Bailey, D., and Wright, E., (2003). Practical SCADA for Industry, IDC Technologies, accessed March 2007.

[18] Boyer, S., (1999). SCADA Supervisory Control and Data Acquisition, $2^{\text {nd }}$ Edition, accessed November 2006. 
[19] James H., Graham, and Waleed H., ElSaid, (2007). N-Secrecy Authentication Response to Graduated Threat Levels in SCADA Networks, S4 SCADA Security Scientific Symposium 2007, Miami, USA, January $24-25$.

[20] American Gas Association (AGA) Report No.12, (2006). Cryptographic Protection of SCADA Communications. Part 1: Background, Policies and Test Plan, accessed January 2007.

[21] Committee on Science and Technology. National Research Council, (2002). Making the Nation Safer: The role of science and technology in countering terrorism, accessed January 2008.

[22] Encryption device planned for legacy SCADA installations: Government Computer News (GCN), accessed Sept 2008.

[23] SCADA vs. The Hackers, (2002) http://memagazine.org/backissues, accessed Sept 2008.

[24] Jeffery L. Hieb, Patricia A. Ralston, and James H. Graham, (2007): Testing of Security Enhanced DNP3 on Operational SCADA System; Intelligent Systems Research Laboratory, Technical Report TR-ISRL-07-01, Dept. of Computer Engineering and Computer Science, University of Louisville, Louisville KY 40292.

[25]http://www.managingautomation.com/maonline/directory/product/GE_Fanuc_Intelli gent_Platforms_Proficy_HMISCADA_IFIX_5832707.

[26] Waleed H. El-said and James H. Graham (2009). Double Secrecy: An Enhanced Cryptographic Approach for SCADA System Security: International conference on Computer Applications in Industry and Engineering. 
[27] RSA Laboratories, What is SSL? http://www.rsa.com/rsalabs/node.asp? id=2293, accessed Jan 2010.

[28] Tom Davis (2003). RSA Encryption.

[29] RSA Laboratories, What is Diffie-Helman? http://www.rsa.com/rsalabs/ node. asp?id=2248, accessed Jan 2010.

[30] Brian Lockwood. The Math and Algorithms Behind Elliptic Curve Cryptography, Department of Mathematics, University of Wisconsin-Eau Claire.

[31] The fundamentals of SCADA (2004). Bentley Systems, Incorporated

[32] Certicom Research (2000). Standards For Efficient Cryptography: Elliptic Curve Cryptography.

[33] Hieb, J.L., Graham, J.H., \& Patel, S.C. (2007). Cyber Security Enhancements for SCADA and DCS Systems. Critical Infrastructure Protection: Issues and Solutions, Springer.

[34] Magali Bardet (2005). On the complexity of a Grobner Basis algorithm. INRIA, (2005). 


\section{Class KeyGenerator}

\section{APPENDIX A}

\section{Code}

$1^{*}$ This class uses the crypto java package class KeyGenerator to generate a DES or triple DES keys and write them into files*

import javax.crypto.*;

public class KeyGenrator

\{

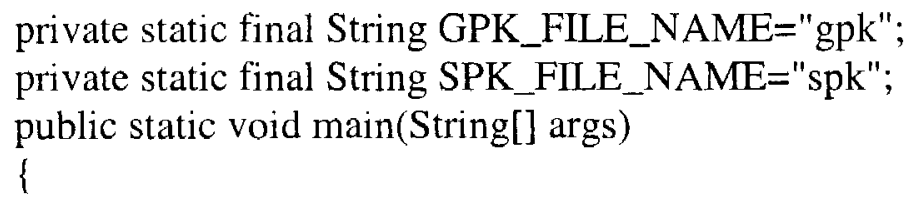

private static final String GPK_FILE_NAME="gpk";

private static final String SPK_FILE_NAME="spk";

public static void main(String[] args)

\{

KeyGenrator kG=new KeyGenrator();

\}

kG.genKey();

$1^{*}$ This function generates the keys and calls writeKeyFile() to write the generated key into a file*/

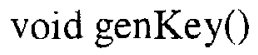

try \{

// Generate a DES key

KeyGenerator keyGen = KeyGenerator.getInstance("DES");

SecretKey gpk = keyGen.generateKey();

writeKeyFile(gpk,GPK_FILE_NAME);

SecretKey spk = keyGen.generateKey();

writeKeyFile(spk,SPK_FILE_NAME);

\} catch (java.security.NoSuchAlgorithmException e)

\{

e.printStackTrace();

1

$l^{*}$ This function encodes the generated key into bytes and write them into the given file name*/

void writeKeyFile(SecretKey key,String fileName)

\{

byte [] keyInBytes=key.getEncoded();

tryl

OutputStreamWriter oSW=new OutputStreamWriter(new

FileOutputStream(fileName));

oSW.write(new String(keyInBytes));

oSW.close();

\}

catch (IOException e) \{

e.printStackTrace();

\} 
\}

\section{Class StringEncrypter}

/* This class does the real job of encryption and decryption of passed message strings. It is to be used by the Unit class */

import java.io.UnsupportedEncodingException;

import java.security.InvalidKeyException;

import java.security.NoSuchAlgorithmException;

import java.security.spec.KeySpec;

import javax.crypto.Cipher;

import javax.crypto.NoSuchPaddingException;

import javax.crypto.SecretKey;

import javax.crypto.SecretKeyFactory;

import javax.crypto.spec.DESKeySpec;

import javax.crypto.spec.DESedeKeySpec;

import sun.misc.BASE64Decoder;

import sun.misc.BASE64Encoder;

public class StringEncrypter

\{

public static final String DESEDE_ENCRYPTION_SCHEME = "DESede";

public static final String DES_ENCRYPTION_SCHEME = "DES";

public static final String DEFAULT_ENCRYPTION_KEY = "This is a fairly

long phrase used to encrypt";

private KeySpec

keySpec;

private SecretKeyFactory keyFactory;

private Cipher

"UTF8";

private static final String UNICODE_FORMAT =

public StringEncrypter( String encryptionScheme ) throws EncryptionException

\{

\}

this( encryptionScheme, DEFAULT_ENCRYPTION_KEY );

I* The constructor takes the encryption scheme which is either DES or triple DES together with the encryption key, converts the key into bytes, constructs the keySpec, constructs the key factory and the cipher to be used in the encryption/decryption */

public StringEncrypter( String encryptionScheme, String encryptionKey )

throws EncryptionException

\{

if ( encryptionKey $==$ null )

throw new IllegalArgumentException( "encryption key was

null" );

if ( encryptionKey.trim().length ()$<24$ )

throw new IllegalArgumentException(

"encryption key was less than 24 characters"

)

try

\{ 


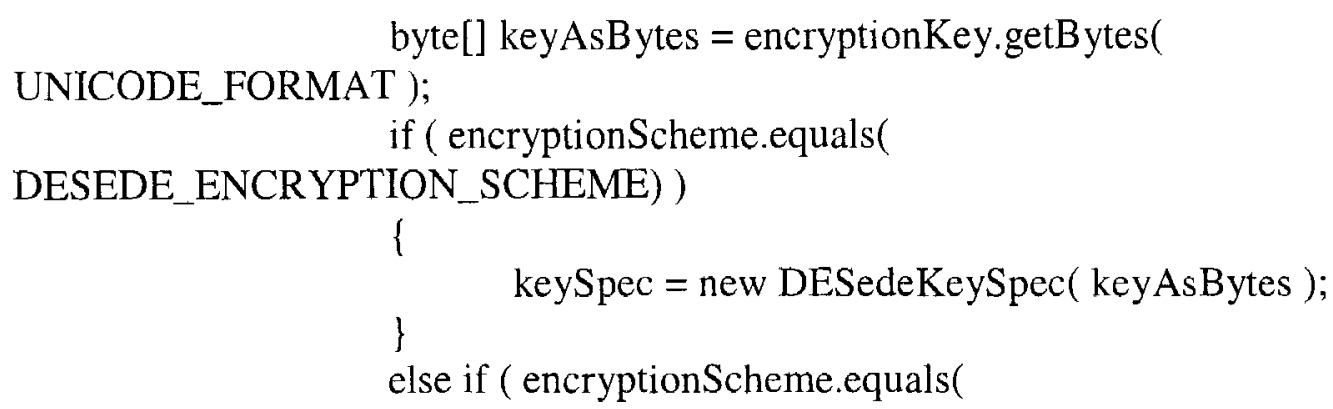

not supported: "

throw new IllegalArgumentException( "Encryption scheme

encryptionScheme );

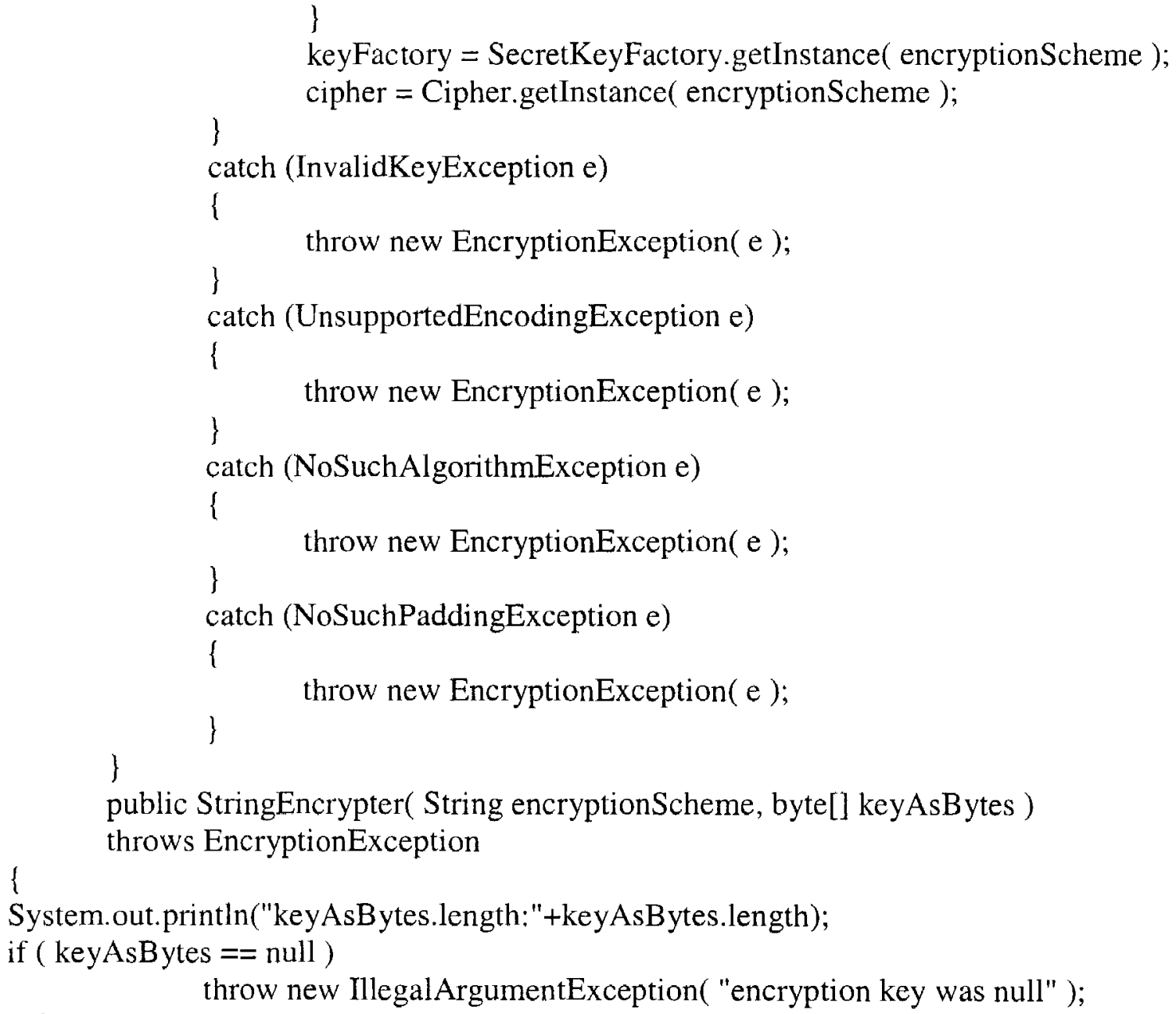




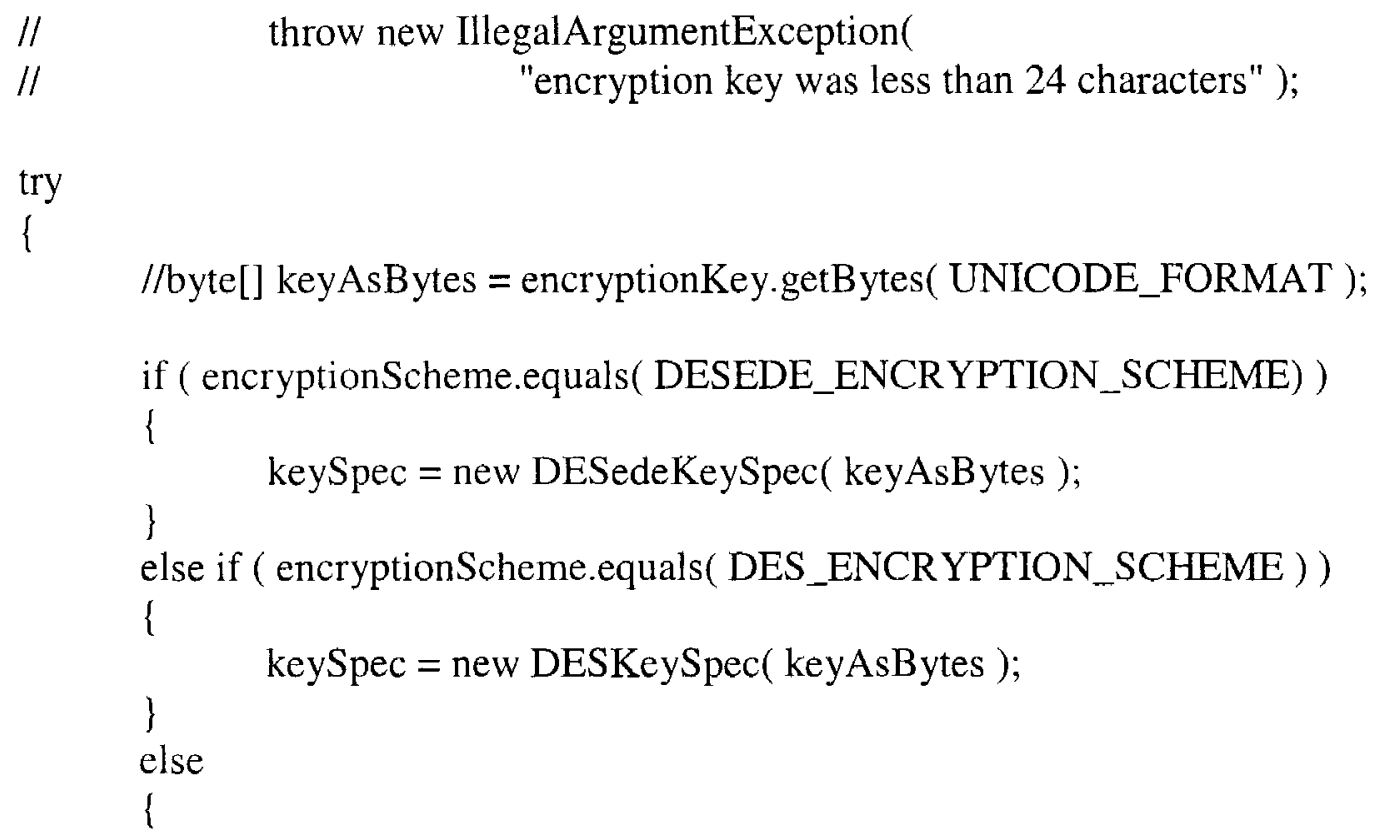
initializes the cipher, and finally uses the base64encooder to encode the cipher text $* /$ public String encrypt( String unencryptedString ) throws EncryptionException

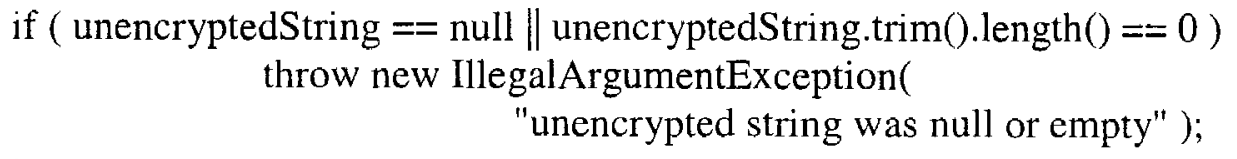


UNICODE_FORMAT );

SecretKey key = keyFactory.generateSecret $($ keySpec $)$;

cipher.init( Cipher.ENCRYPT_MODE, key);

byte[ $]$ cleartext $=$ unencryptedString.getBytes (

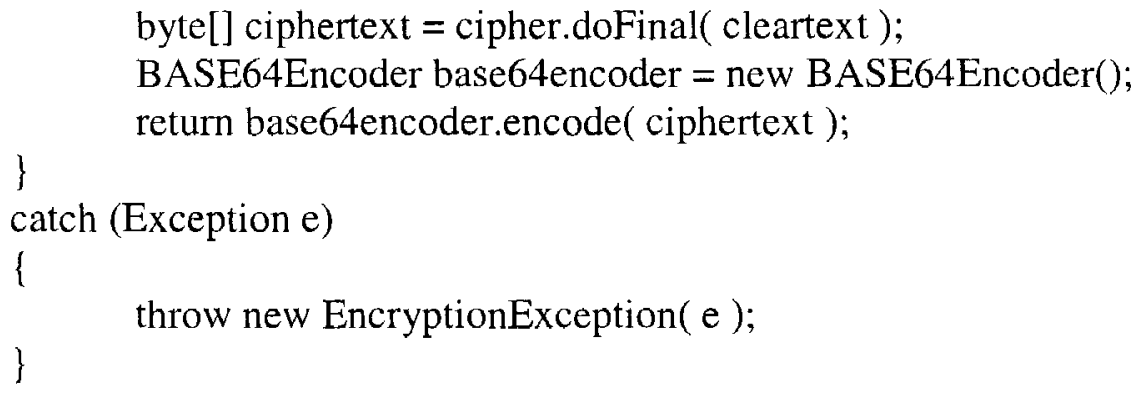

I* This method uses the base64decoder to decode the given encrypted string*/ public String decrypt( String encryptedString) throws EncryptionException

if ( encryptedString $==$ null $\|$ encryptedString.trim().length ()$<=0$ ) throw new IllegalArgumentException( "encrypted string

was null or empty" );

try

\{

SecretKey key = keyFactory.generateSecret ( keySpec ); cipher.init( Cipher.DECRYPT_MODE, key );

BASE64Decoder base64decoder = new BASE64Decoder(); byte[] cleartext $=$ base64decoder.decodeBuffer( encryptedString ); byte[] ciphertext $=$ cipher doFinal $($ cleartext $)$;

\} return bytes 2 String( ciphertext );

catch (Exception e)

\{

throw new EncryptionException( $\mathrm{e}$ );

\}

$1 *$ This is a simple converter function from an array of bytes into a string*/ private static String bytes 2 String( byte[] bytes )

\{

StringBuffer stringBuffer $=$ new StringBuffer();

for (int $\mathrm{i}=0 ; \mathrm{i}<$ bytes.length; $\mathrm{i}++$ )

\{

stringBuffer.append( (char) bytes[i] );

\}

return stringBuffer.toString();

\}

public static class EncryptionException extends Exception \{ 


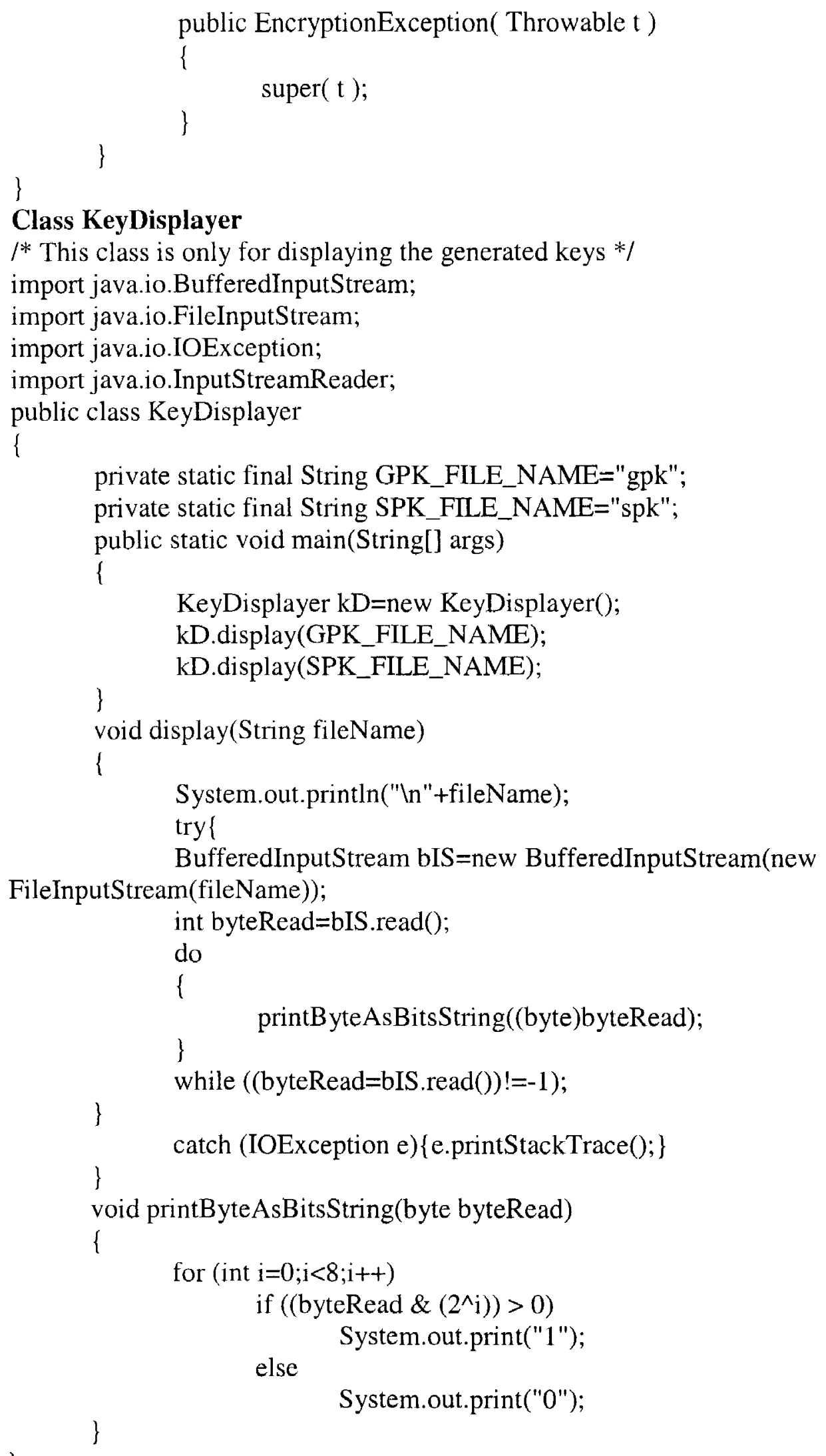




\section{Class Unit (with no security)}

$1 *$ This is the main visitor class that is to be called from the MTU and the RTU to handle all the functions of different authentication algorithms - that version of the Unit class doesn't have any authentication implementation though */ import java.io.*; import java.util.ArrayList; import java.util.Hashtable; import nsecrecy.StringEncrypter.EncryptionException; public class Unit

\{

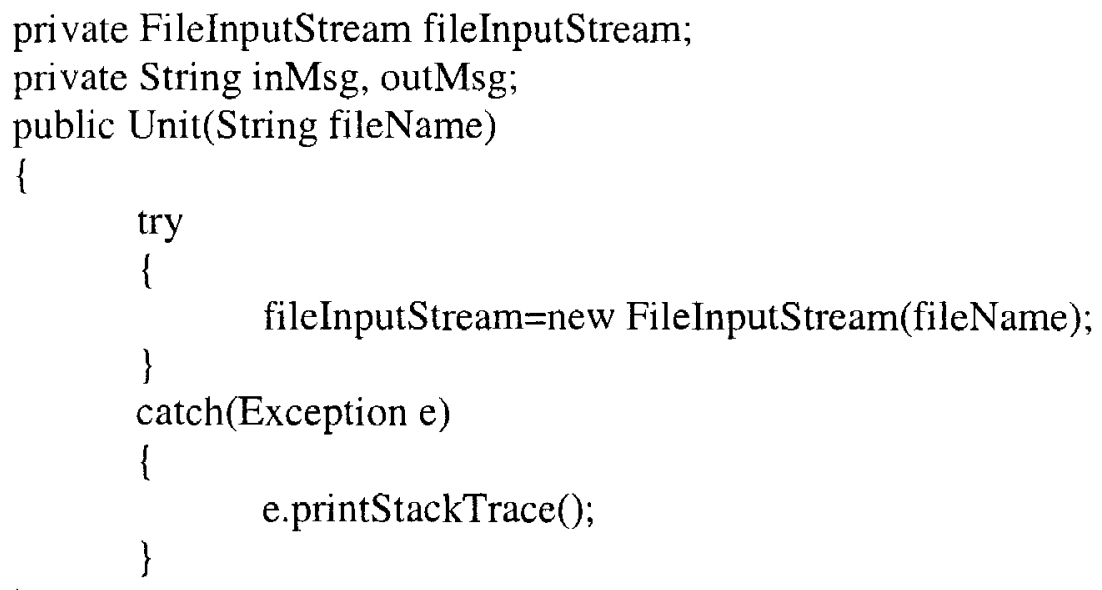




\section{Class MTU (with no security)}

$1 *$ This class represents the master terminal unit with DNP3 protocol*/

package noSecurity;

import java.io.BufferedWriter;

import java.io.ByteArrayInputStream;

import java.io.File;

import java.io.IOException;

import java.io.PrintWriter;

import java.io.StreamTokenizer;

import java.net.Socket;

import java.net.UnknownHostException;

public class MTU

\{

public static int port;

public static int ERROR;

public static String server;

public static Socket socket $=$ null;

public static ByteArrayInputStream input ;

public static PrintWriter output;

public static final int MAX_FRAG_SIZE $=300$; //Eventually, change to 2048

public static byte[] MTUfragment = new byte[MAX_FRAG_SIZE]; // DNP

Fragment octets to be sent to RTU.

public static byte[] fragFromRTU = new byte[MAX_FRAG_SIZE]; // DNP

Fragment octets for response from RTU.

public static int offset;

public static int length;

public static int byteToBeSent;

public static StreamTokenizer tokenizer;

public static int last;

public static boolean fileWrite_wait_state = false;

public static long lastModifiedTime;

public static long newModifiedTime;

public static long currentTime;

public static File clientInputFile = new File ("INPUT.dat");

public static boolean IsUnsolicitedMsgReceived = false;

public static int contentValue1;

public static int contentValue2;

public static int contentValue3;

public static PrintWriter fileContents;

public static File outputFile;

public static long firstStamp;

public static long secondStamp;

public static long timeDifference;

public static long totalTimeDifference;

public static String fileName = "OUTPUT.dat" ; 
public static BufferedWriter out;

static Unit mtuUnit;

public static void main(String[] args)

\{

long time $1=$ System.currentTimeMillis();

System.out.println("MTU Time1:"+time1);

server $=" 10.202 .2 .67 "$;// for socket connections

port $=2000$;

// use the unit object to do the message preparation

mtuUnit=new Unit("INPUT.DAT");

mtuUnit.prepareMsg();

connect(); // connect to the RTU

prepareToSend();

sendArray();// Send the message array

\}

$I^{*}$ Initiates a socket connection with the server IP/Port*I

public static void connect()

\{

try

socket $=$ new Socket(server, port $)$;

System.out.println("Port:"+port);

System.out.println("Connected with RTU " + socket.getInetAddress ()$+$ ":" + socket.getPort() + "ln");

\}

catch (UnknownHostException e) \{

System.out.println(e);

\}

catch (IOException e) \{

System.out.println(e);

\}

\}

$1 *$ Stacks a message fragment into a byte array and transmits it into the server*/ public static void prepareToSend()

\{

try \{

int offset $=0$;

int length $=$ mtuUnit.getMsg().length();

MTUfragment=mtuUnit.getMsg().getBytes();

input $=$ new ByteArrayInputStream(MTUfragment, offset, length);

$/ /$ get input array and transmit it to server

byteToBeSent $=$ input.read () ;

output $=$ new PrintWriter(socket.getOutputStream(),true);

\}

catch (IOException e) \{

System.out.println(e); 


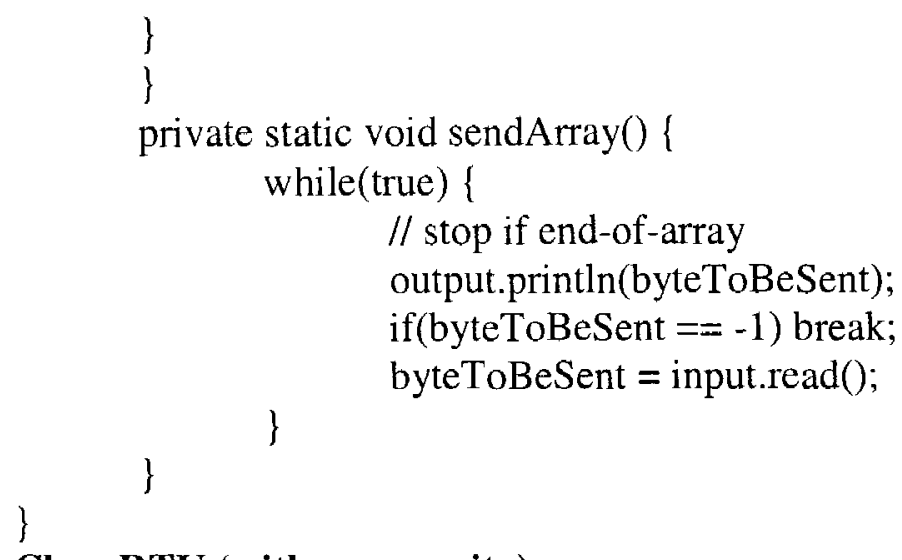

public static int port; public static ServerSocket server_socket; public static Socket socket; public static StreamTokenizer tokenizer; public static final int MAX_FRAG_SIZE $=10000 ;$ //Eventually, change to 2048 public static byte[] fragFromMTU = new byte[MAX_FRAG_SIZE];

// Fragment received from MTU public static byte[] responseFrag = new byte[MAX_FRAG_SIZE];

//Fragment to send to MTU public static File newfile; public static FileInputStream outputFile; public static BufferedWriter out; public static ByteArrayInputStream ACKtoMTU;

public static PrintWriter outputAsACK;

public static String message; public static int byteACK; public static String fileName; 
public static int contentValue; public static PrintWriter fileContents; public static PrintWriter unsolicitedOutput; public static boolean keepListening = true; public static FileWriter newWriteFile; static Unit rtuUnit; static int len $=0$; public static void main(String[] args)

\{

// The port to listen at for a socket connection port $=2000$; rtuUnit=new Unit("OUTPUT.DAT");

try \{ \} getSocket(); // Sit and listen for any message from MTU catch (Exception e) \{ System.out.println("Socket Problems"); System.out.println(e);

receiveMTUfrag();

long time2=System.currentTimeMillis(); System.out.println("RTU time :"+time2);

\} rtuUnit.setMsg(new String(fragFromMTU,0,len));

// Wait and respond to socket connections public static void getSocket() \{

try \{

server_socket $=$ new ServerSocket(port);

System.out.println("port:"+port);

System.out.println("RTU waiting for request on port " + server_socket.getLocalPort( $)+$ " .....");

socket $=$ server_socket.accept();

System.out.println("New connection accepted from: "+ socket.getInetAddress() + ":" + socket.getPort());

\} catch (IOException e) \{ System.out.println("Socket Error"); System.out.println(e);

\} \}

// Receive the message fragment from MTU public static void receiveMTUfrag()// Get the information sent by MTU \{ int byteToBeSent $=0$; 
initializeArray(); //Create an empty fragment to receive MTU octets prepareStream(); //Start reading token stream sent by MTU

\}

public static void initializeArray() //Initialize the fragment array \{

for (int $\mathrm{i}=0 ; \mathrm{i}<$ fragFromMTU.length; $i++$ ) fragFromMTU $[\mathrm{i}]=0 \mathrm{x} 0$;

//initialize array

\}

public static void prepareStream() //Get info from the stream sent by MTU \{

try \{

tokenizer $=$ new StreamTokenizer ( new InputStreamReader(socket.getInputStream()));

if (tokenizer.nextToken ()$==$ tokenizer.TT_NUMBER) \{ fillArray(); //Fill the entire fragment array with the info sent by

MTU

\} else \{

socket. $\ln ")$;

System.out.println("Nothing to read from MTU in the

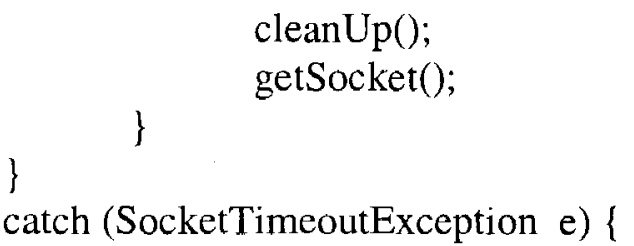

System.out.println("InNo MTU request received in last five

seconds, so");

try \{

$\operatorname{try}\{\quad$ socket.setSoTimeout(0);
\} catch (SocketException e2)\{

System.out.println("Socket Timedout Here");

System.out.println(e2);

\}

\}

catch (SocketException e) \{

System.out.println("In"+e);

create new socket.");

System.out.println("Client has closed the connection. RTU will

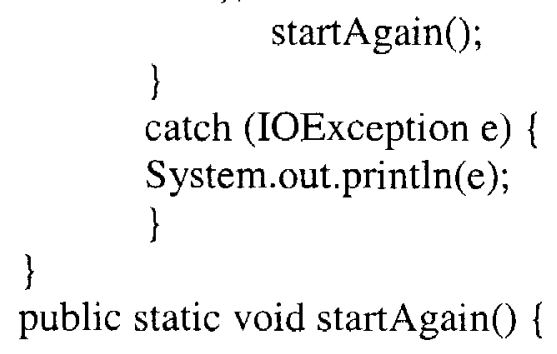


keepListening = false;

//System.out.println("In Will clean up and start listening again" ); cleanUp();

try \{

getSocket(); // Sit and listen for any message from MTU

\}

catch (Exception e)

\{

System.out.println("Socket Problems");

\}

System.out.println(e);

while (true)

\{

receiveMTUfrag(); // Get the information sent by MTU

\}

\} //end while-loop

public static void fillArray()

//Populate the fragment array with info from MTU

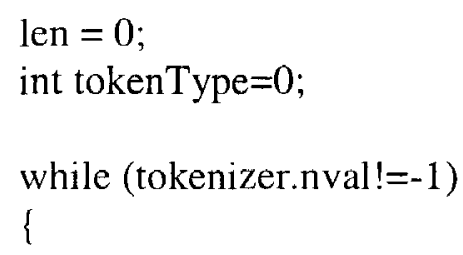

try 1

fragFromMTU[len] = (byte)tokenizer.nval;

if $($ len $==55)$;

//Let's get the contents of the file MTU wants us to

create.

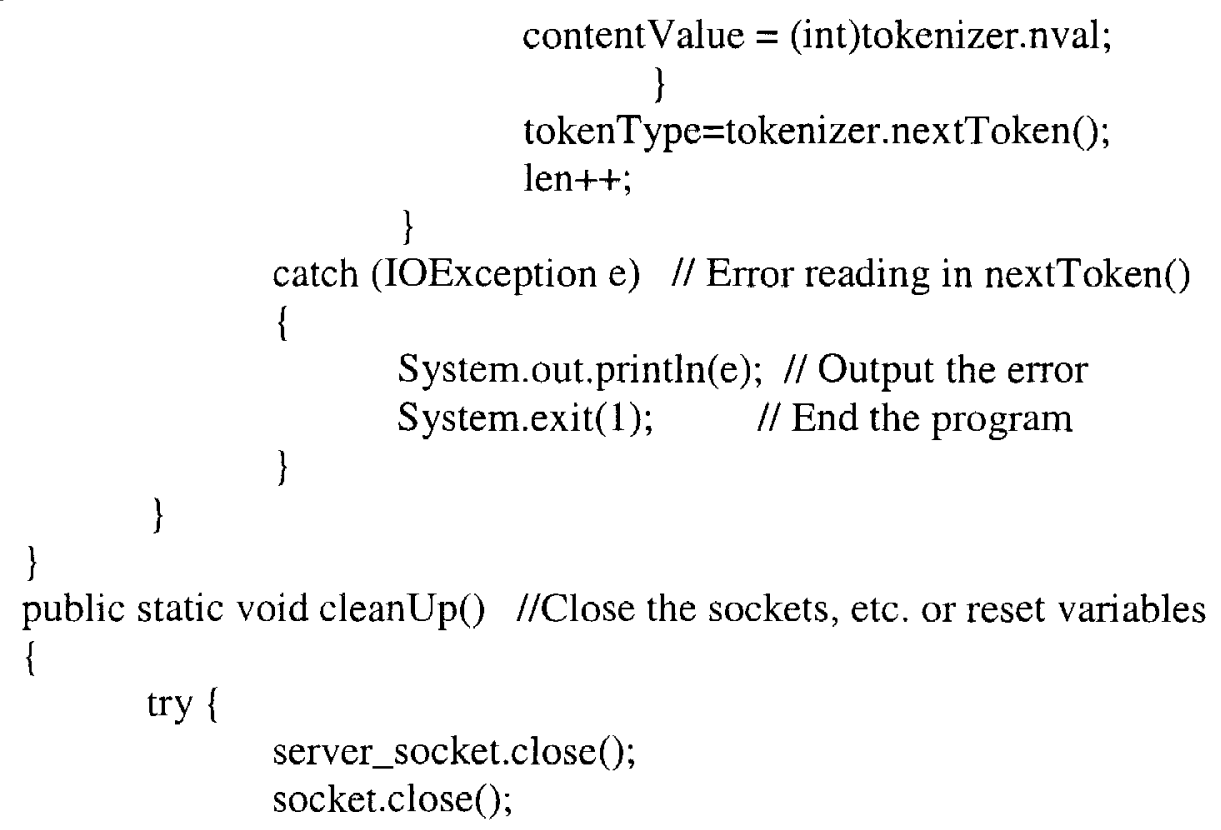

public static void cleanUp() //Close the sockets, etc. or reset variables \{ 
System.out.println("RTU is closing the socket now

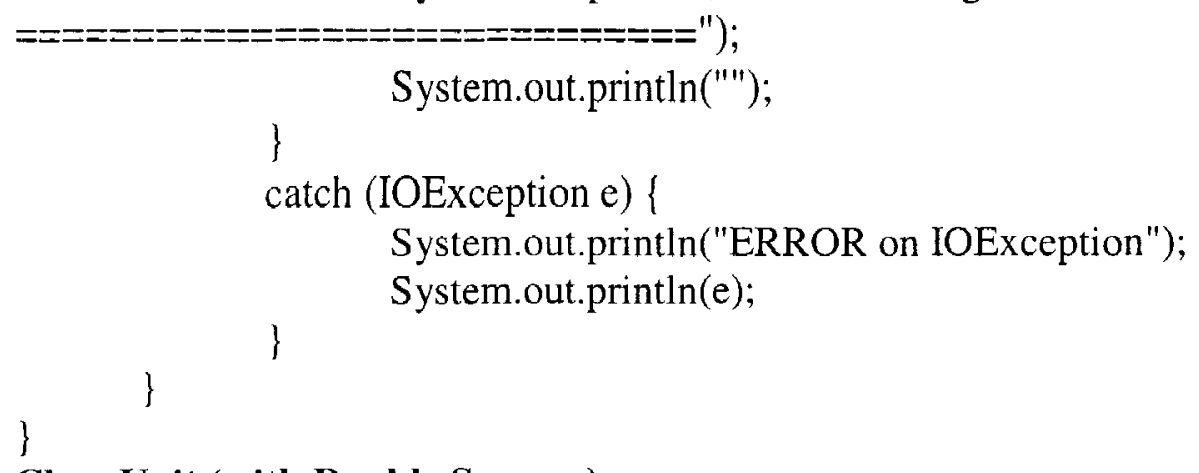

1* The visitor implementation of the unit class with the double secrecy implemented.

There will be only two secrets here, ssec1, and ssec2, and two corresponding keys, gpk, and $\mathrm{spk} * /$

package nsecrecy;

import java.io.*;

import java.util.ArrayList;

import java.util.Hashtable;

import nsecrecy.StringEncrypter.EncryptionException;

public class Unit

private static final String GPK_FILE_NAME="gpk";

private static final String SPK_FILE_NAME="spk";

private static final String SSEC1_FILE_NAME="ssec1"; private static final String SSEC2_FILE_NAME="ssec2"; private static String gpkEncKey;

private static String spkEncKey;

private static String ssec 1;

private static String ssec2;

$/ /$ to serve as a delimiter in the final concatenated message

private static String endMark="//";

// DES or triple DES

private String encryptionScheme $=$

StringEncrypter.DESEDE_ENCRYPTION_SCHEME;

private StringEncrypter encrypterGPK,encrypterSPK;

private FileInputStream fileInputStream;

private String inMsg, outMsg;

// The hashtable containing keys and secrets as the double secrecy algorithm

private Hashtable hTable=new Hashtable();

public Unit(String fileName)

\{

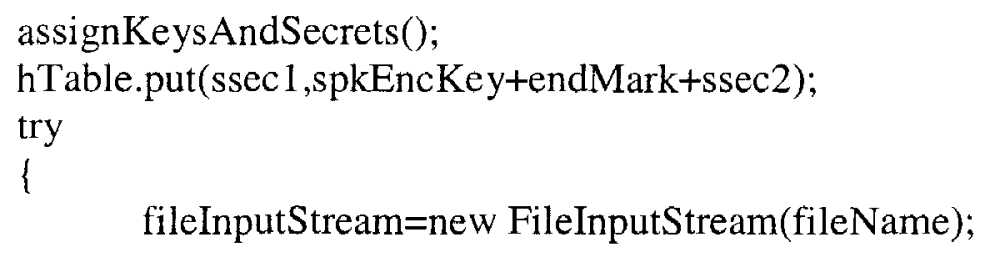

fileInputStream=new FileInputStream(fileName); 


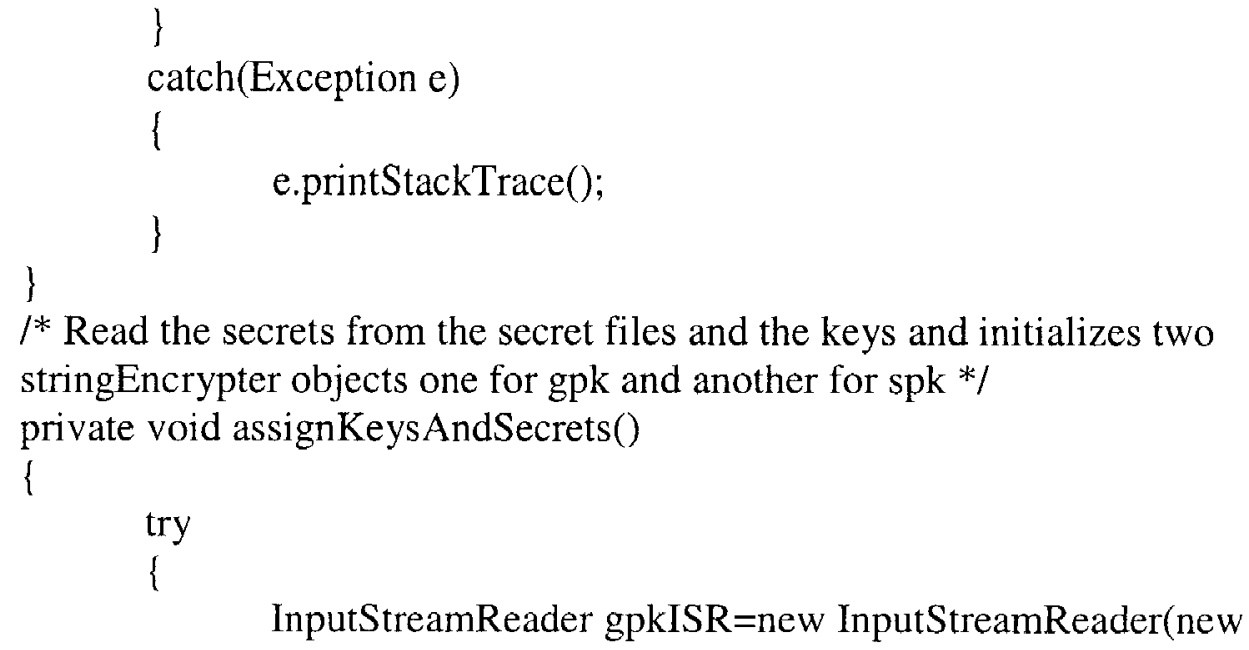

FileInputStream(GPK_FILE_NAME));

InputStreamReader spkISR=new InputStreamReader(new

FileInputStream(SPK_FILE_NAME)); InputStreamReader ssec1ISR=new InputStreamReader(new

FileInputStream(SSEC1_FILE_NAME));

InputStreamReader ssec2ISR=new InputStreamReader(new

FileInputStream(SSEC2_FILE_NAME));

char []cbuf = new char[1000];

int len=gpkISR.read(cbuf);

gpkEncKey=String.copyValueOf(cbuf,0,len);

len=spkISR.read(cbuf);

spkEncKey=String.copyValueOf(cbuf,0,len);

len=ssec l ISR.read (cbuf);

ssec $1=$ String.copyValueOf(cbuf,0,len);

len=ssec2ISR.read(cbuf);

$\operatorname{ssec} 2=$ String.copyValueOf $($ cbuf, 0, len $)$;

encrypterGPK = new

StringEncrypter(encryptionScheme,gpkEncKey );

encrypterSPK = new

StringEncrypter(encryptionScheme,spkEncKey );

catch (Exception e)

\{

e.printStackTrace();

\}

)

String getMsg()

\{

return outMsg;

\}

void setMsg(String msg)

\{ 
inMsg=msg;
$1 *$ Prepares the message to be sent by concatenating the encrypted ssec logether with the encrypted message and the encrypted ssec2, according to the double secrecy algorithm */

void prepareMsg()

\{

InputStreamReader isr=new InputStreamReader(fileInputStream); char [] cbuf $=$ new char $[1000]$;

try

\{

int len=isr.read(cbuf); outMsg=String.copyValueOf(cbuf,0,len);

System.out.println("content of file:"+outMsg);

String header=encrypt(ssec1,encrypterGPK);

String footer=encrypt(ssec2,encrypterSPK);

String body=encrypt(outMsg,encrypterSPK);

outMsg=header+endMark+body+endMark+footer;

\}

catch (Exception e)

\{

\}

e.printStackTrace();

\}

/*Calling the encrypt function of the stringEncrypter object*/

String encrypt(String content,StringEncrypter encrypter)

\{

String ret="Enc Error";

try \{

ret= encrypter.encrypt(content);

\}

catch (EncryptionException e)

1

e.printStackTrace();

\}

return ret;

\}

$1 *$ Calling the decrypt function of the stringEncrypter object*/

String decrypt(String content,StringEncrypter encrypter)

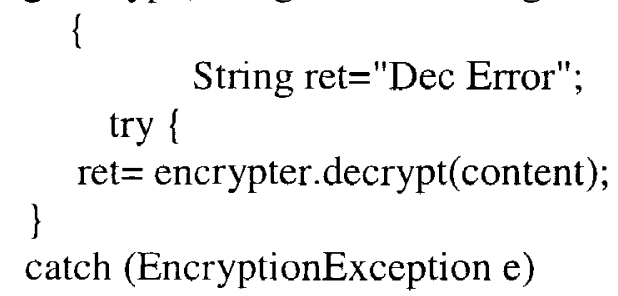




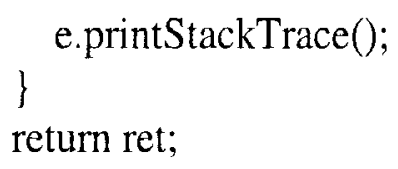

/* Authenticating the message according to the double secrecy algorithm*/ boolean authenticateMsg()

boolean ret=false;

String encHeader=inMsg.substring(0,inMsg.index Of(endMark));

String

temp=inMsg.substring(inMsg.indexOf(endMark)+endMark.length());

String encBody=temp.substring $(0$,temp.indexOf(endMark));

String

encFooter=temp.substring(temp.indexOf(endMark)+endMark.length());

String header=decrypt(encHeader,encrypterGPK);

if (hTable.containsKey(header))

\{

System.out.println("Passed first test");

String listValue $=($ String $)$ hTable.get $($ header $)$;

String spk=listValue.substring( 0 ,listValue.indexOf(endMark));

String

ssec2=listValue.substring(listValue.indexOf(endMark)+endMark.length());

String footer=decrypt(encFooter,encrypterSPK);

if (footer.equals(ssec2))

\{

System.out.println("Passed second test"); ret=true;

String msgContent=decrypt(encBody,encrypterSPK);

System.out.println("Authenticated and msg content

is:"+msgContent);

$$
\begin{aligned}
& \text { else } \\
& \text { els }
\end{aligned}
$$

System.out.println("Ah Oh");

\}

return ret;

\}

\}

\section{Class MTU (with Double Secrecy)}

/* Same MTU implementation as above*/

package nsecrecy;

import java.io.BufferedWriter;

import java.io.ByteArrayInputStream;

import java.io.File;

import java.io.IOException;

import java.io.PrintWriter;

import java.io.StreamTokenizer; 
import java.net.Socket;

import java.net.UnknownHostException;

public class MTU

\{

public static int port;

public static int ERROR;

public static String server;

public static Socket socket $=$ null;

public static ByteArrayInputStream input ;

public static PrintWriter output;

public static final int MAX_FRAG_SIZE = 300; //Eventually, change to 2048

public static byte[] MTUfragment = new byte[MAX_FRAG_SIZE]; // DNP

Fragment octets to be sent to RTU.

public static byte[] fragFromRTU = new byte[MAX_FRAG_SIZE]; // DNP

Fragment octets for response from RTU.

public static int offset;

public static int length;

public static int byteToBeSent;

public static StreamTokenizer tokenizer;

public static int last;

public static boolean fileWrite_wait_state $=$ false;

public static long lastModifiedTime;

public static long newModifiedTime;

public static long currentTime;

public static File clientInputFile = new File ("INPUT.dat");

public static boolean IsUnsolicitedMsgReceived = false;

public static int contentValuel;

public static int contentValue2;

public static int contentValue3;

public static PrintWriter fileContents;

public static File outputFile;

public static long firstStamp;

public static long secondStamp;

public static long timeDifference;

public static long totalTimeDifference;

public static String fileName = "OUTPUT.dat" ;

public static BufferedWriter out;

static Unit mtuUnit;

public static void main(String[] args)

\{

server $=" 10.202 .2 .67 "$;

port $=2000$;

mtuUnit=new Unit("INPUT.DAT");

mtuUnit.prepareMsg();

connect();

long time $1=$ System.current TimeMillis(); 
// System.out.println("MTU Time1:"+time1);

prepareToSend();

sendArray();

long time2=System.currentTimeMillis();

System.out.println("Time elapsed is:"+(time2-time1));

$$
\text { \} }
$$

public static void connect()

\{

try \{

socket = new Socket(server, port);

System.out.println("Port:"+port);

System.out.println("Connected with RTU " + socket.getInetAddress()+ ":" + socket.getPort ()$+$ "In");

\}

catch (UnknownHostException e) \{

System.out.println(e);

\}

catch (IOException e)

System.out.println(e);

\}

\}

public static void prepareToSend()

i

try \{

int offset $=0$;

int length $=$ mtuUnit.getMsg () .length () ;

MTUfragment=mtuUnit.getMsg().getBytes();

input = new ByteArrayInputStream(MTUfragment, offset, length);

// get input array and transmit it to server

byteToBeSent $=$ input.read();

output $=$ new PrintWriter $($ socket.getOutputStream (), true $)$;

\}

catch (IOException e) \{

System.out.println(e);

\}

\}

private static void sendArray() \{

while(true) \{

// stop if end-of-array

output.println(byteToBeSent);

if $($ byteToBeSent $==-1)$ break;

\}

byteToBeSent = input.read () ; 
\}

\section{Class RTU (with Double Secrecy)}

$1^{*}$ The same implementation of RTU as above with the exception of calling the

Unit.authenticate() method to authenticate the other party *I

package nsecrecy;

import java.io.BufferedWriter;

import java.io.ByteArrayInputStream;

import java.io.File;

import java.io.FileInputStream;

import java.io.FileWriter;

import java.io.IOException;

import java.io.InputStreamReader;

import java.io.PrintWriter;

import java.io.StreamTokenizer;

import java.net.ServerSocket;

import java.net.Socket;

import java.net.SocketException;

import java.net.SocketTimeoutException;

public class RTU

\{

public static int port;

public static ServerSocket server_socket;

public static Socket socket;

public static StreamTokenizer tokenizer;

public static final int MAX_FRAG_SIZE $=10000$; //Eventually, change to 2048

public static byte[] fragFromMTU = new byte[MAX_FRAG_SIZE]; // Fragment received from MTU

public static byte[] responseFrag = new byte[MAX_FRAG_SIZE]; //Fragment to send to MTU

public static File newfile;

public static FileInputStream outputFile;

public static BufferedWriter out;

public static ByteArrayInputStream ACKtoMTU;

public static PrintWriter outputAsACK;

public static String message;

public static int byteACK;

public static String fileName;

public static int contentValue;

public static PrintWriter fileContents;

public static PrintWriter unsolicitedOutput;

public static boolean keepListening = true;

public static FileWriter newWriteFile;

static Unit rtuUnit;

static int len $=0$;

public static void main(String[] args) 


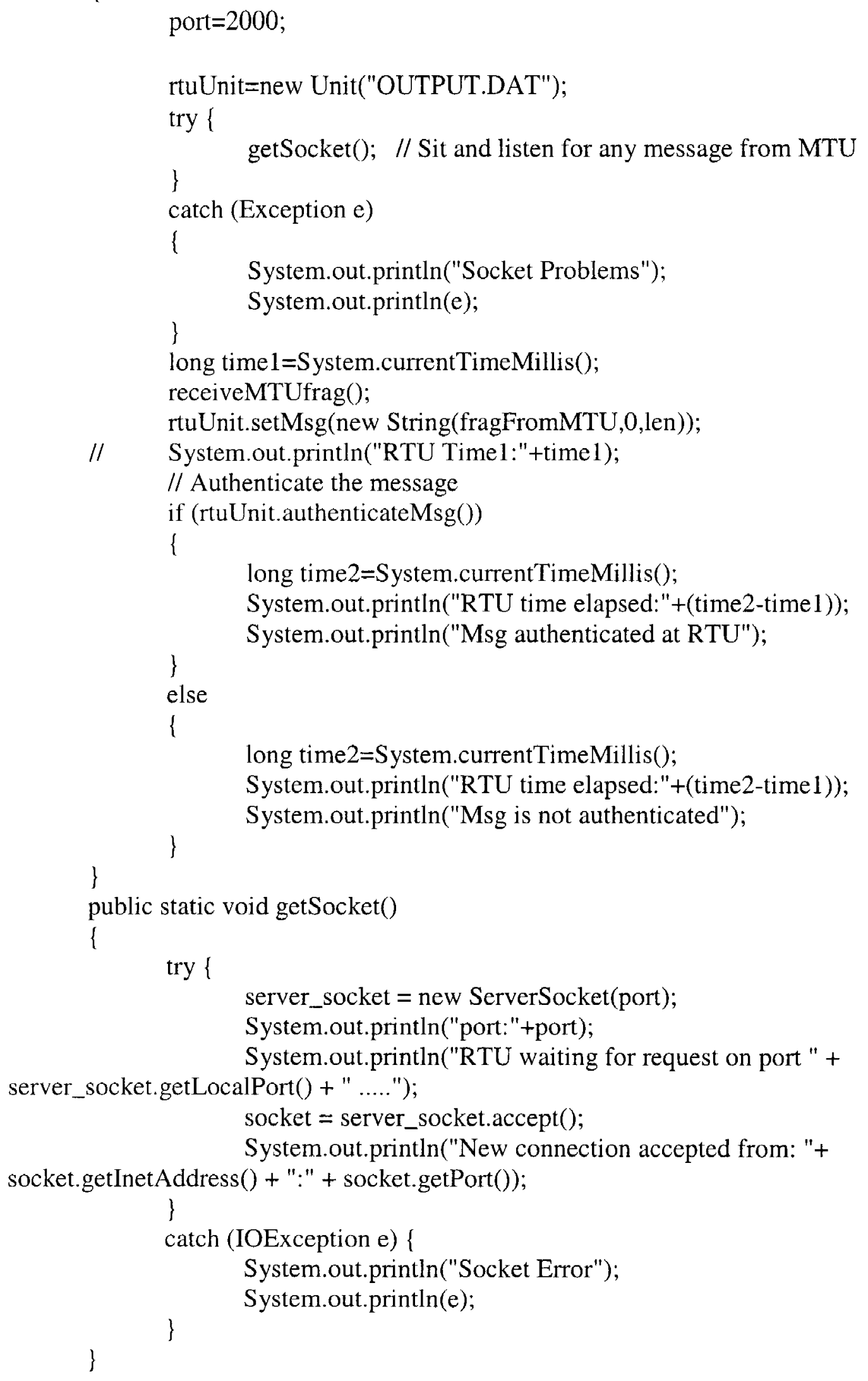


public static void receiveMTUfrag() // Get the information sent by MTU \{

int byteToBeSent $=0$;

initializeArray(); //Create an empty fragment to receive MTU octets

prepareStream(); //Start reading token stream sent by MTU

\}

public static void initializeArray() //Initialize the fragment array

$$
\text { i }
$$

//initialize array

for (int $\mathrm{i}=0 ; \mathrm{i}<$ fragFromMTU.length; $\mathrm{i}++$ ) fragFromMTU[i] = 0x0;

\}

public static void prepareStream() //Get info from the stream sent by MTU \{

try \{

tokenizer $=$ new StreamTokenizer (

new InputStreamReader(socket.getInputStream()));

if $($ tokenizer.nextToken ()$==$ tokenizer.TT_NUMBER) \{

fillArray(); //Fill the entire fragment array with the info sent by MTU

\}

else \{

socket.ln ");

System.out.println("Nothing to read from MTU in the

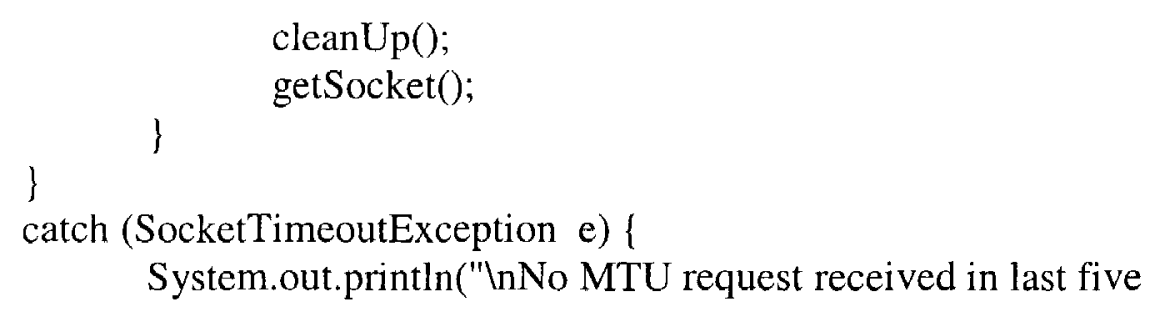


\}

public static void startAgain() \{

keepListening $=$ false;

//System.out.println("In Will clean up and start listening again" ); cleanUp();

try \{

getSocket(); // Sit and listen for any message from MTU

\}

catch (Exception e)

\{

System.out.println("Socket Problems");

System.out.println(e);

\}

while (true)

\{

receiveMTUfrag(); // Get the information sent by MTU

\} //end while-loop

\}

public static void fillArray() //Populate the fragment array with info from MTU \{

len $=0$;

int tokenType $=0$;

while (tokenizer.nval!=-1)

\{

try \{

fragFromMTU[len] = (byte)tokenizer.nval;

if $($ len $==55)\{$

//Let's get the contents of the file MTU wants us to

create.

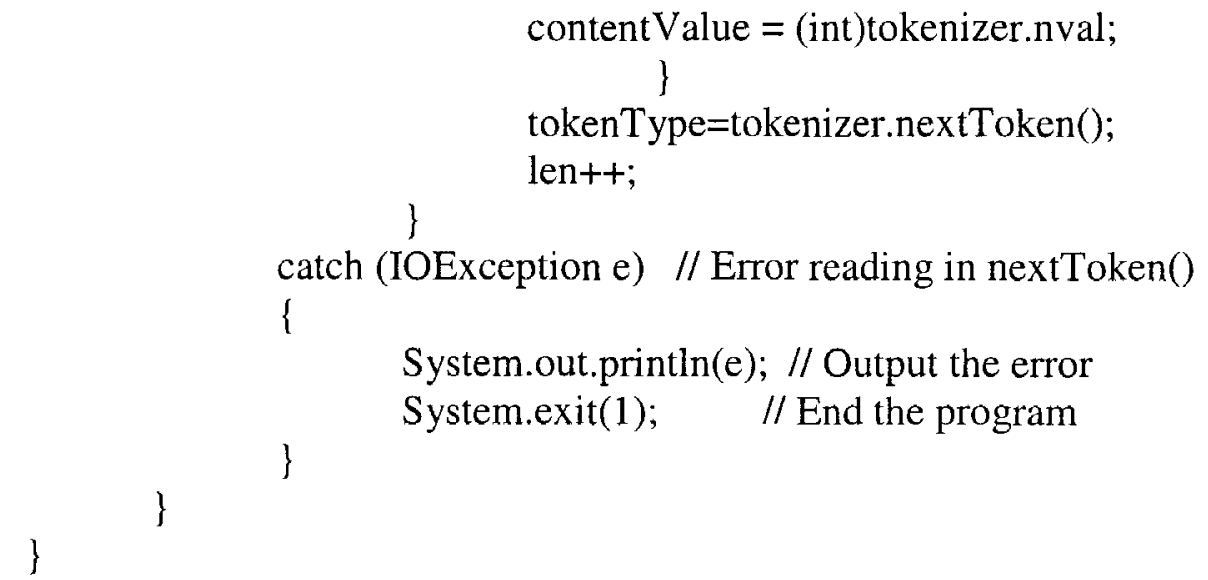

public static void cleanUp() //Close the sockets, etc. or reset variables

try \{

server_socket.close(); 


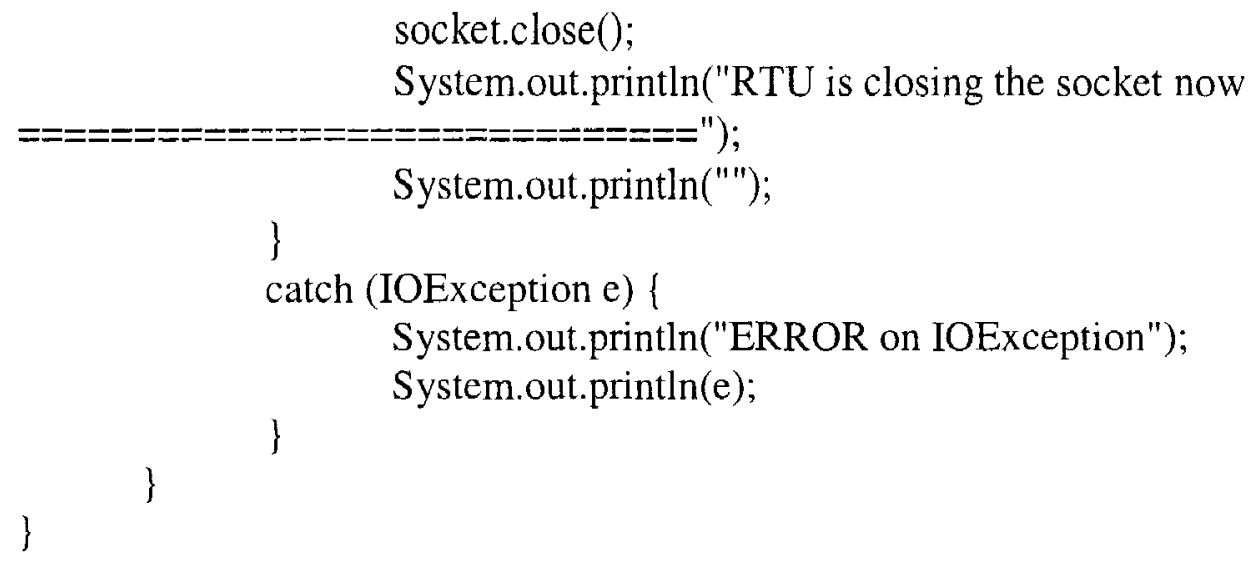

socket.close();

System.out.println("RTU is closing the socket now

\section{Class Unit (with N Secrecy)}

$1 *$ This class represents the visitor implementation of the $\mathrm{N}$-Secrecy authentication algorithm */ package nsecrecyUpgrade; import java.io.*; import java.util.ArrayList; import java.util.Hashtable; import nsecrecyUpgrade.StringEncrypter.EncryptionException; public class Unit \{

//The prefixes of the keys and the secrets' names private static final String ENCKEY_FILE_NAME_PRE="enckey"; private static final String SSEC_FILE_NAME_PRE="ssec"; private String[] encKey=new String[5]; private String[] ssec=new String[5];

I* The delimiter for the concatenated string composing the message to be sent */ private static String endMark="//";

1* The number of secrets to be used as the $\mathrm{N}$ in the $\mathrm{N}$-secrecy*/ private int securityLevel $=2$; private FileInputStream fileInputStream; private String inMsg, outMsg; private Hashtable hTable=new Hashtable(); public Unit(String fileName,int securityLevel) \{

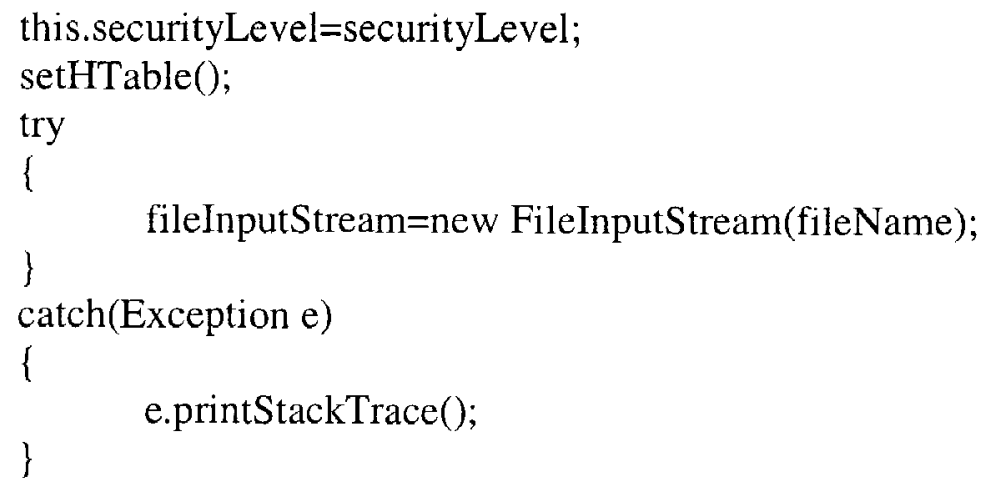


\}

/* filling the hashtable like the $\mathrm{N}$-secrecy algorithm*/

private void setHTable()

\{

if (securityLevel $>1$ )

for (int $\mathrm{i}=0 ; \mathrm{i}<$ securityLevel; $i++$ )

\{

assignKeysAndSecrets(i);

if $(\mathrm{i}>0)$

hTable.put(ssec[i-1],encKey[i]+endMark+ssec[i]);

\}

\}

$I^{*}$ Reading the secret file and the key file with the given index and filling the enckey and the ssec arrays. This method is called in a loop from setHTable ()$^{*} /$

private void assignKeys AndSecrets(int $\mathrm{ndx}$ )

\{

try

\{

InputStreamReader keyISR=new InputStreamReader(new

FileInputStream(ENCKEY_FILE_NAME_PRE+ndx));

InputStreamReader ssecISR=new InputStreamReader(new

FileInputStream(SSEC_FILE_NAME_PRE+ndx));

char []cbuf = new char[1000];

int len=keyISR.read(cbuf);

encKey[ndx]=String.copyValueOf(cbuf,0,len);

len=ssecISR.read(cbuf);

ssec[ndx]=String.copyValueOf(cbuf,0,len);

\}

catch (Exception e)

\{

\}

e.printStackTrace();

\}

String getMsg()

\{

return outMsg;

\}

void setMsg(String msg)

\{

inMsg=msg;

\}

$1^{*}$ Preparing the message to be sent. It has the same logic as the one in the double secrecy implementation, except for setting up the footer which will be in a loop depending on the security level in the method setupFooter() below */ void prepareMsg()

\{ 
InputStreamReader is $=$ =new InputStreamReader(fileInputStream); char []cbuf = new char[1000];

try

\{

int len=isr.read(cbuf);

outMsg=String.copyValueOf(cbuf,0,len);

System.out.println("content of file:"+outMsg);

String header=encrypt(ssec[0],encKey[0]);

String footer=setUpFooter();

String body=encrypt(outMsg,encKey[securityLevel-1]); outMsg=header+endMark+body+endMark+footer;

\}

catch (Exception e)

\{

e.printStackTrace();

\}

private String setUpFooter()

\{

String footer="";

for (int $i=1 ; i<$ securityLevel $; i++$ )

\{

String temp=encrypt(ssec[i],encKey[i]);

\}

footer=footer+temp+endMark;

return footer;

\}

// Same as the one in the double secrecy

String encrypt(String content,String key)

\{

String ret="Enc Error";

String encryptionScheme $=$

StringEncrypter.DESEDE_ENCRYPTION_SCHEME;

StringEncrypter encrypter;

System.out.println("Key:"+key);

try \{

encrypter = new StringEncrypter(encryptionScheme,key $)$;

\}

ret= encrypter.encrypt(content);

catch (EncryptionException e)

\{

e.printStackTrace();

\}

return ret;

\}

// Same as the one in the double secrecy 
String decrypt(String content,String key)

\{

$$
\text { String ret="Dec Error"; }
$$

String encryptionScheme $=$

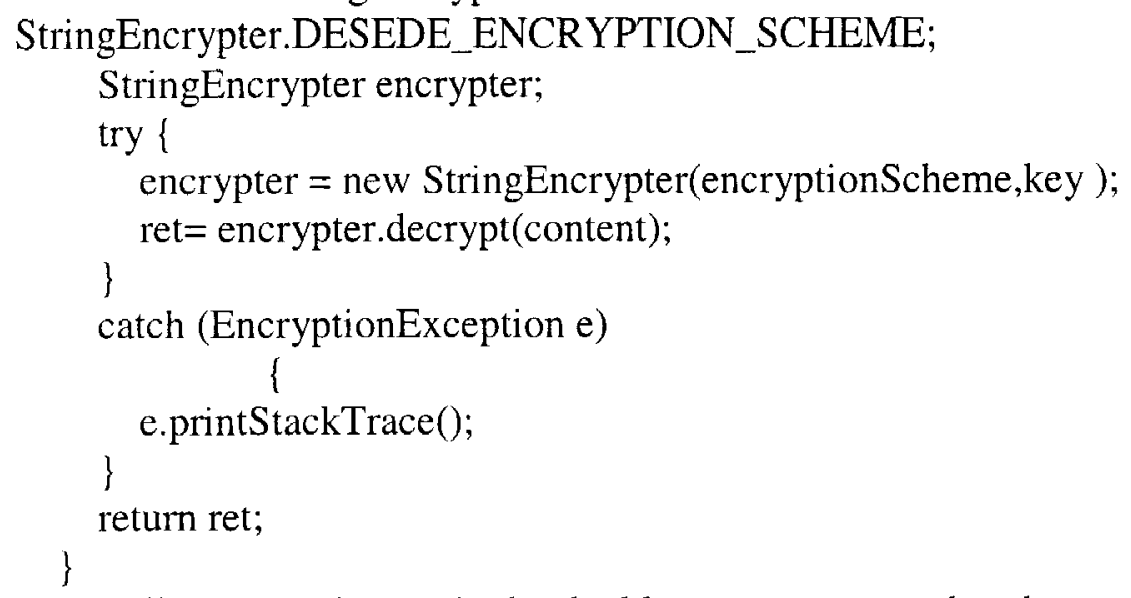

// Same as the one in the double secrecy except that the tests are being checked in a loop depending on the security level, to check if the hashtable contains the $\operatorname{ssec}[i]$ secret as a key where $i$ is the secret/key index boolean authenticateMsg()

boolean ret=true;

String $\mathrm{aPk}=" "$;

String aSsec="";

String encHeader=inMsg.substring $(0$,inMsg.indexOf(endMark));

String

temp=inMsg.substring(inMsg.indexOf(endMark)+endMark.length());

String encBody=temp.substring $(0$,temp.indexOf(endMark));

String encFooter=temp. substring(temp.indexOf(endMark)+endMark.length());

String header $=$ decrypt (encHeader,encKey[0]);

if (hTable.containsKey(header))

\{

System.out.println("Passed lookup test no:1");

String list Value $=($ String $)$ hTable.get(header); aPk=listValue.substring $(0$, listValue.indexOf(endMark)); aSsec=listValue.substring(listValue.indexOf(endMark)+endMark.length()); for(int $\mathrm{i}=0 ; \mathrm{i}<$ securityLevel- $1 ; \mathrm{i}++$ )

\{

$$
\text { if }(\mathrm{i}>0)
$$

if (hTable.containsKey(ssec[i]))

no:"+(i+1));

System.out.println("Passed lookup test

listValue $=($ String $)$ hTable.get(ssec[i]); 
aPk=listValue.substring $(0$,listValue.indexOf(endMark));

aSsec=listValue.substring(listValue.indexOf(endMark)+endMark.length());

\}

else return false;

String

aFooter=encFooter substring $(0$,encFooter.indexOf(endMark $)$ );

encFooter=encFooter.substring(encFooter.indexOf(endMark)+endMark.length()); if ((decrypt(aFooter,aPk)).equals(aSsec))

else

System.out.println("Passed match test no:"+(i+1));

return false;

\}

\}

String msgContent=decrypt(encBody,aPk);

System.out.println("Authenticated and msg content is:"+msgContent); return true;

\}

Class MTU (with N Secrecy)

${ }^{*}$ Same as the MTU implementation for the double secrecy except for the new method prepareAndSend (), which is actually preparing the message as the previous

implementation but now we 're calling with more than one security level to test out the functionality and time*/

package nsecrecyUpgrade;

import java.io.BufferedWriter;

import java.io.ByteArrayInputStream;

import java.io.File;

import java.io.IOException;

import java.io.PrintWriter;

import java.io.StreamTokenizer;

import java.net.Socket;

import java.net.UnknownHostException;

public class MTU

\{

public static int port;

public static int ERROR;

public static String server;

public static Socket socket $=$ null;

public static ByteArrayInputStream input ;

public static PrintWriter output;

public static final int MAX_FRAG_SIZE $=300$;

//Eventually, change to 2048

public static byte[] MTUfragment = new byte[MAX_FRAG_SIZE]; // DNP

Fragment octets to be sent to RTU. 
public static byte[] fragFromRTU = new byte[MAX_FRAG_SIZE]; // DNP Fragment octets for response from RTU.

public static int offset;

public static int length;

public static int byteToBeSent;

public static StreamTokenizer tokenizer;

public static int last;

public static boolean fileWrite_wait_state = false;

public static long lastModifiedTime;

public static long newModifiedTime;

public static long currentTime;

public static File clientInputFile = new File ("INPUT.dat");

public static boolean IsUnsolicitedMsgReceived = false;

public static int contentValue1;

public static int contentValue2;

public static int contentValue3;

public static PrintWriter fileContents;

public static File outputFile;

public static long firstStamp;

public static long secondStamp;

public static long timeDifference;

public static long totalTimeDifference;

public static String fileName = "OUTPUT.dat" ;

public static BufferedWriter out;

static Unit mtuUnit;

public static void main(String[] args)

i

server $=" 10.202 .2 .67 "$;

port $=2000$;

connect();

prepareAndSend("INPUT2.DAT",2);

prepareAndSend("INPUT5.DAT",5);

prepareAndSend("INPUT3.DAT",3);

\}

private static void prepareAndSend(String fileName, int securityLevel)

\{

mtuUnit=new Unit(fileName, securityLevel);

mtuUnit.prepareMsg();

long time1=System.currentTimeMillis();

prepareToSend();

sendArray();

long time2=System.currentTimeMillis();

System.out.println("Time elapsed is:"+(time2-time1));

\}

public static void connect()

\{ 


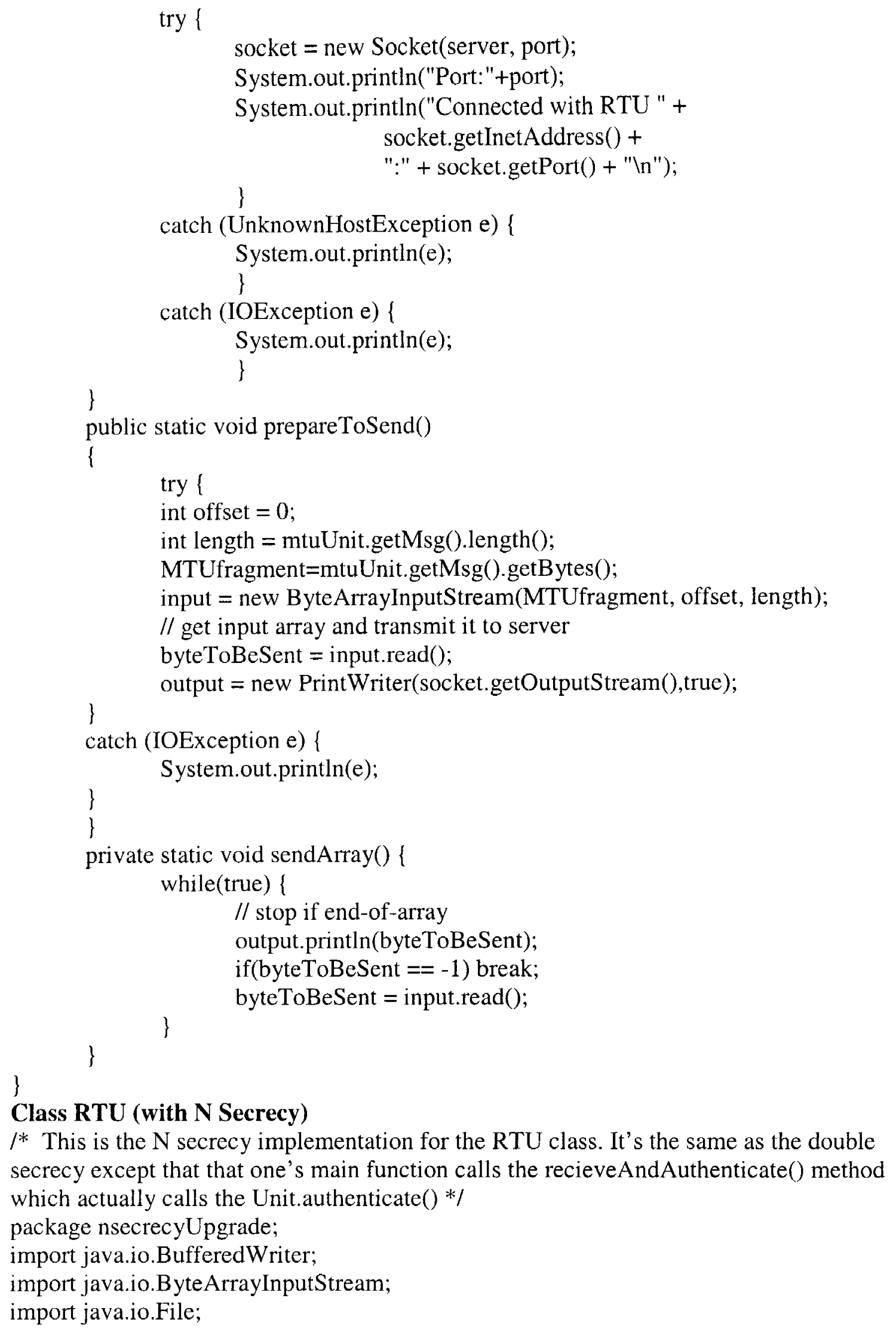

Class RTU (with N Secrecy)

/* This is the N secrecy implementation for the RTU class. It's the same as the double secrecy except that that one's main function calls the recieveAndAuthenticate() method which actually calls the Unit.authenticate ()$*$ I

package nsecrecyUpgrade; import java.io.BufferedWriter; import java.io.ByteArrayInputStream; import java.io.File; 
import java.io.FileInputStream;

import java.io.FileWriter;

import java.io.IOException;

import java.io.InputStreamReader;

import java.io.PrintWriter;

import java.io.StreamTokenizer;

import java.net.ServerSocket;

import java.net.Socket;

import java.net.SocketException;

import java.net.SocketTimeoutException;

public class RTU

\{

public static int port;

public static ServerSocket server_socket;

public static Socket socket;

public static StreamTokenizer tokenizer;

public static final int MAX_FRAG_SIZE $=10000 ;$ //Eventually, change to 2048 public static byte[] fragFromMTU = new byte[MAX_FRAG_SIZE]; // Fragment received from MTU

public static byte[] responseFrag = new byte[MAX_FRAG_SIZE]; //Fragment to send to MTU

public static File newfile;

public static FileInputStream outputFile;

public static BufferedWriter out;

public static ByteArrayInputStream ACKtoMTU;

public static PrintWriter outputAsACK;

public static String message;

public static int byteACK;

public static String fileName;

public static int contentValue;

public static PrintWriter fileContents;

public static PrintWriter unsolicitedOutput;

public static boolean keepListening = true;

public static FileWriter newWriteFile;

static Unit rtuUnit;

static int len $=0$;

public static void main(String[] args)

\{

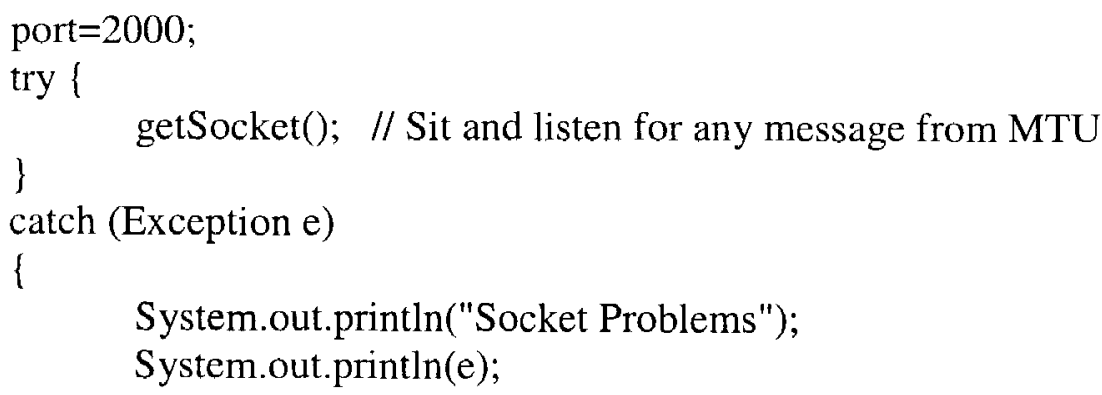

System.out.println("Socket Problems");

System.out.println(e); 


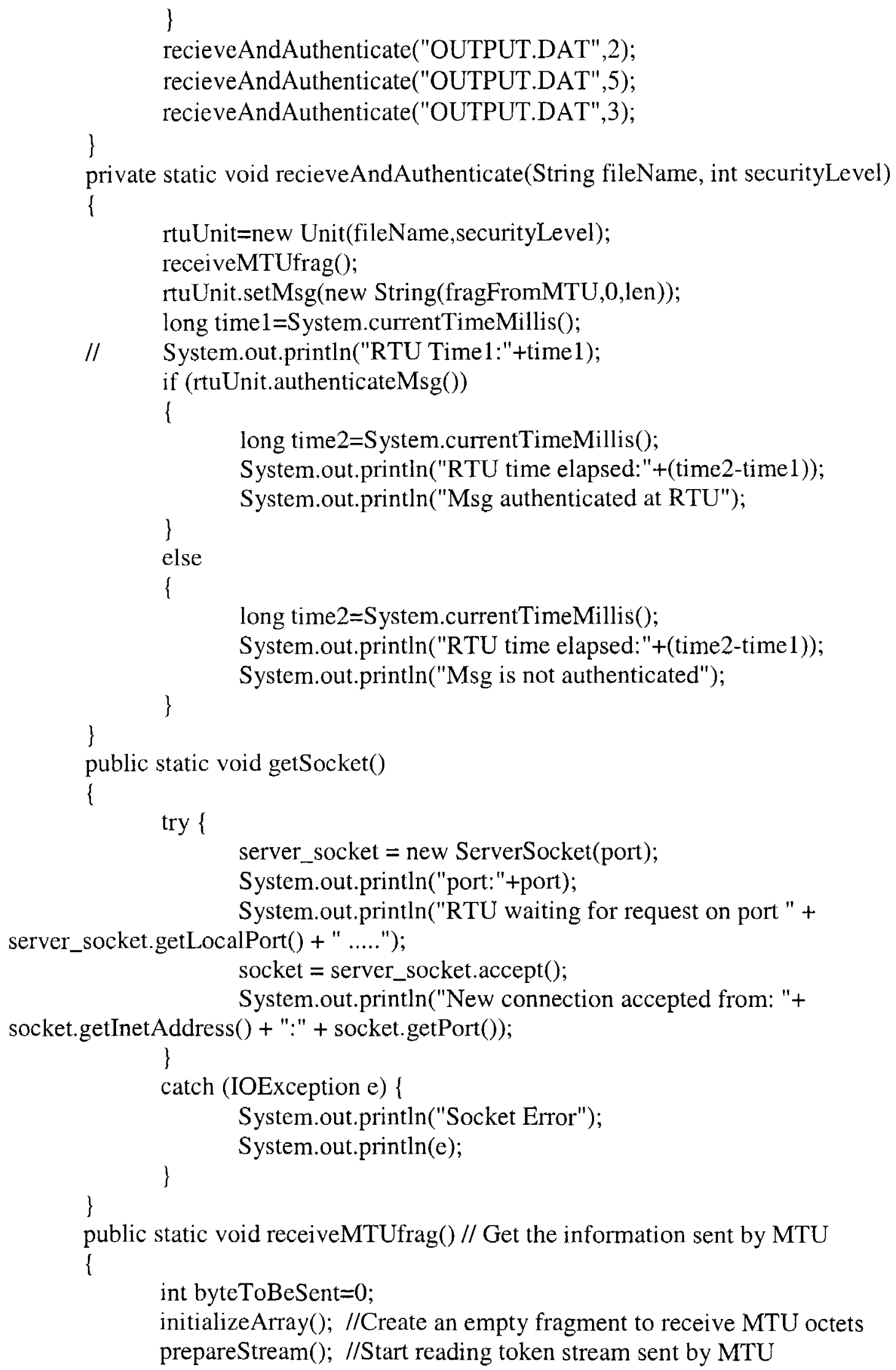


\} public static void initializeArray() //Initialize the fragment array \{

$$
\text { for (int } \mathrm{i}=0 ; \mathrm{i}<\text { fragFromMTU.length; } \mathrm{i}++ \text { ) fragFromMTU[i] = 0x0; }
$$

\section{//initialize array}

\}

public static void prepareStream() //Get info from the stream sent by MTU \{

$\operatorname{try}\{$

$$
\text { tokenizer }=\text { new StreamTokenizer }(
$$
new InputStreamReader(socket.getInputStream())); if (tokenizer.nextToken ()$==$ tokenizer.TT_NUMBER) \{

MTU

fillArray(); //Fill the entire fragment array with the info sent by \} else 1 System.out.println("Nothing to read from MTU in the socket.In ");

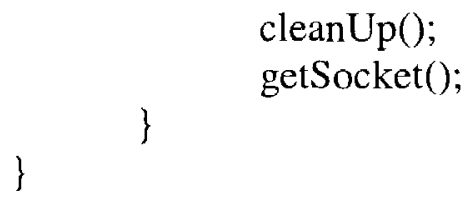

$/ /$

/I

try 1

socket.setSoTimeout( $(0)$;

\}

catch (SocketException e2) \{

System.out.println("Socket Timedout Here");

\} System.out.println(e2);

catch (SocketException e) \{

System.out.println("In"+ e);

create new socket.");

System.out.println("Client has closed the connection. RTU will

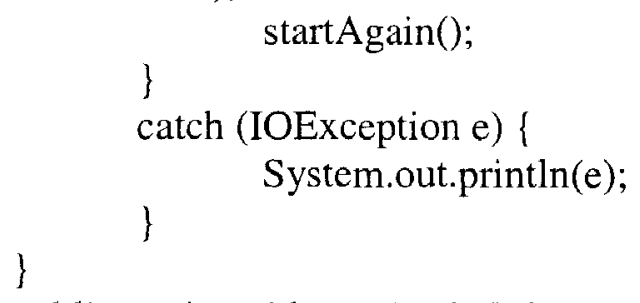




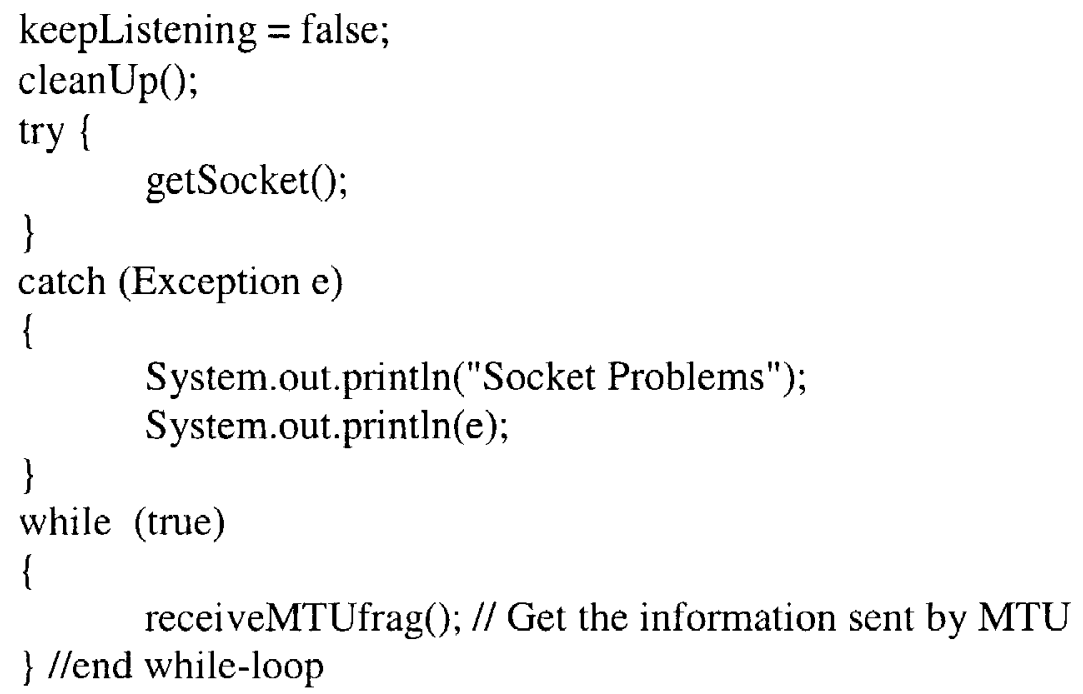

public static void fillArray() //Populate the fragment array with info from MTU

\{

$$
\begin{aligned}
& \text { len }=0 ; \\
& \text { int token Type }=0
\end{aligned}
$$

while (tokenizer.nval!=-1)

i

$\operatorname{try}\{$

fragFromMTU[len] = (byte)tokenizer.nval;

if $($ len $==55)$;

//Let's get the contents of the file MTU wants us to

create.

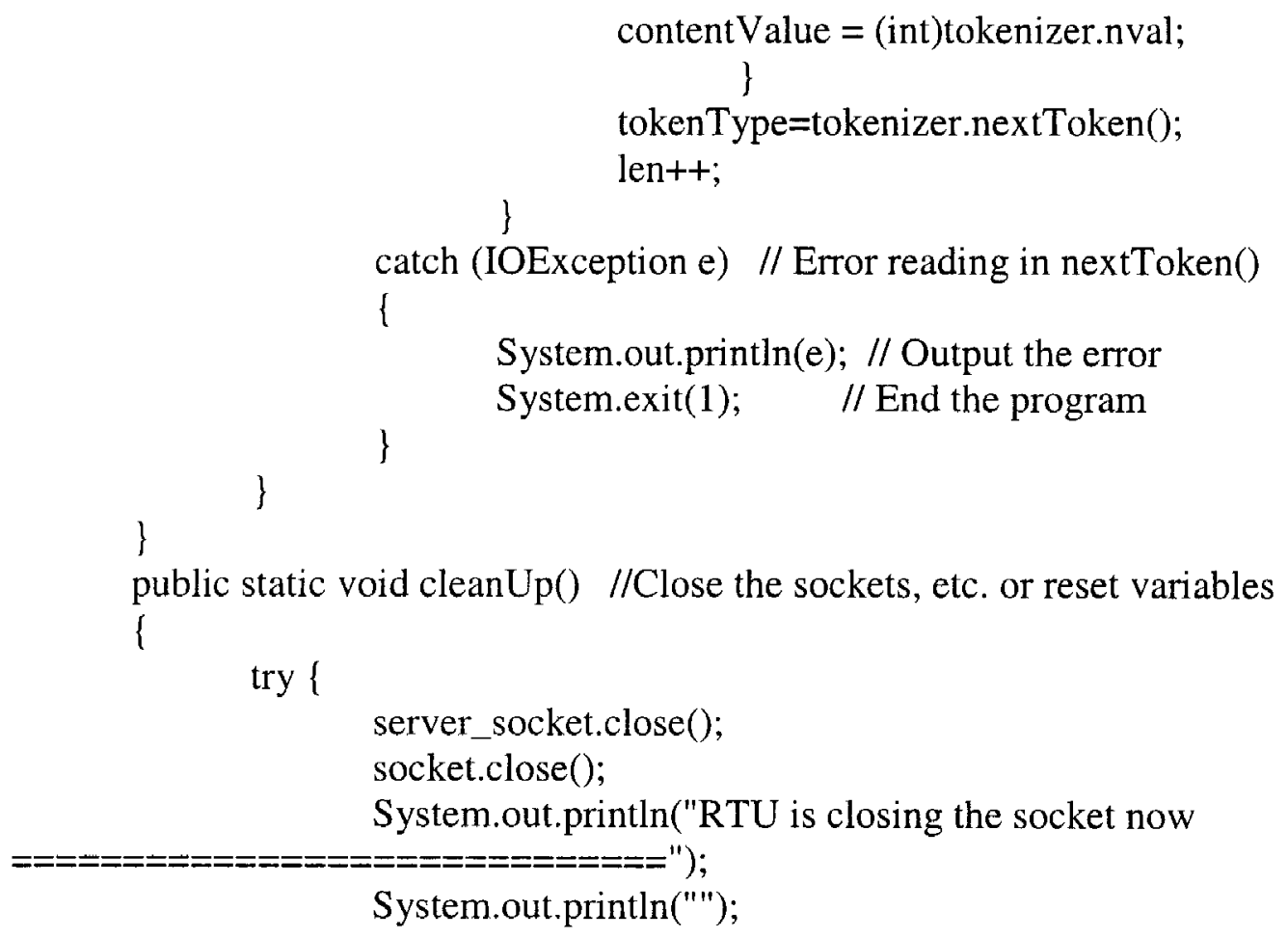




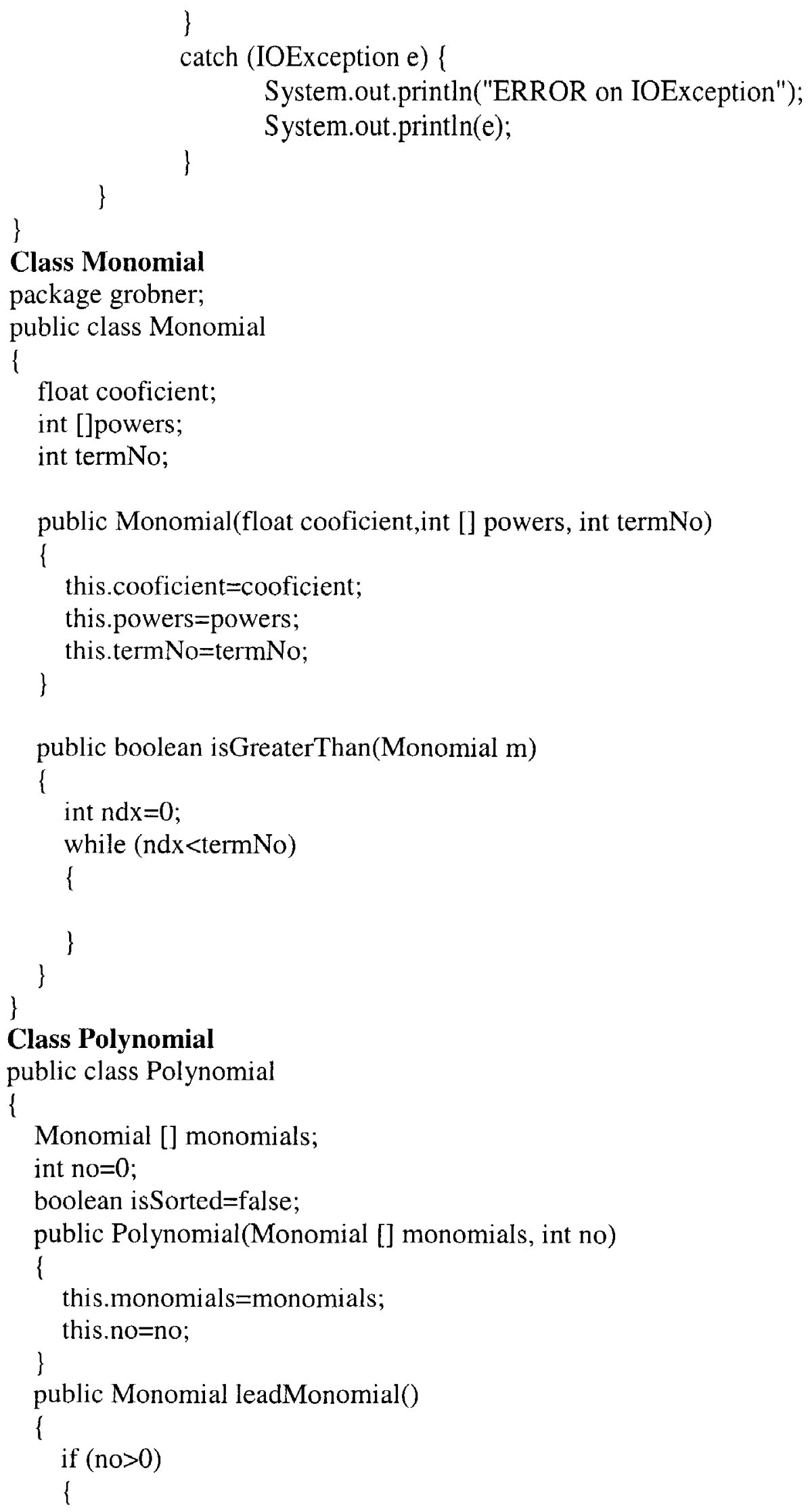




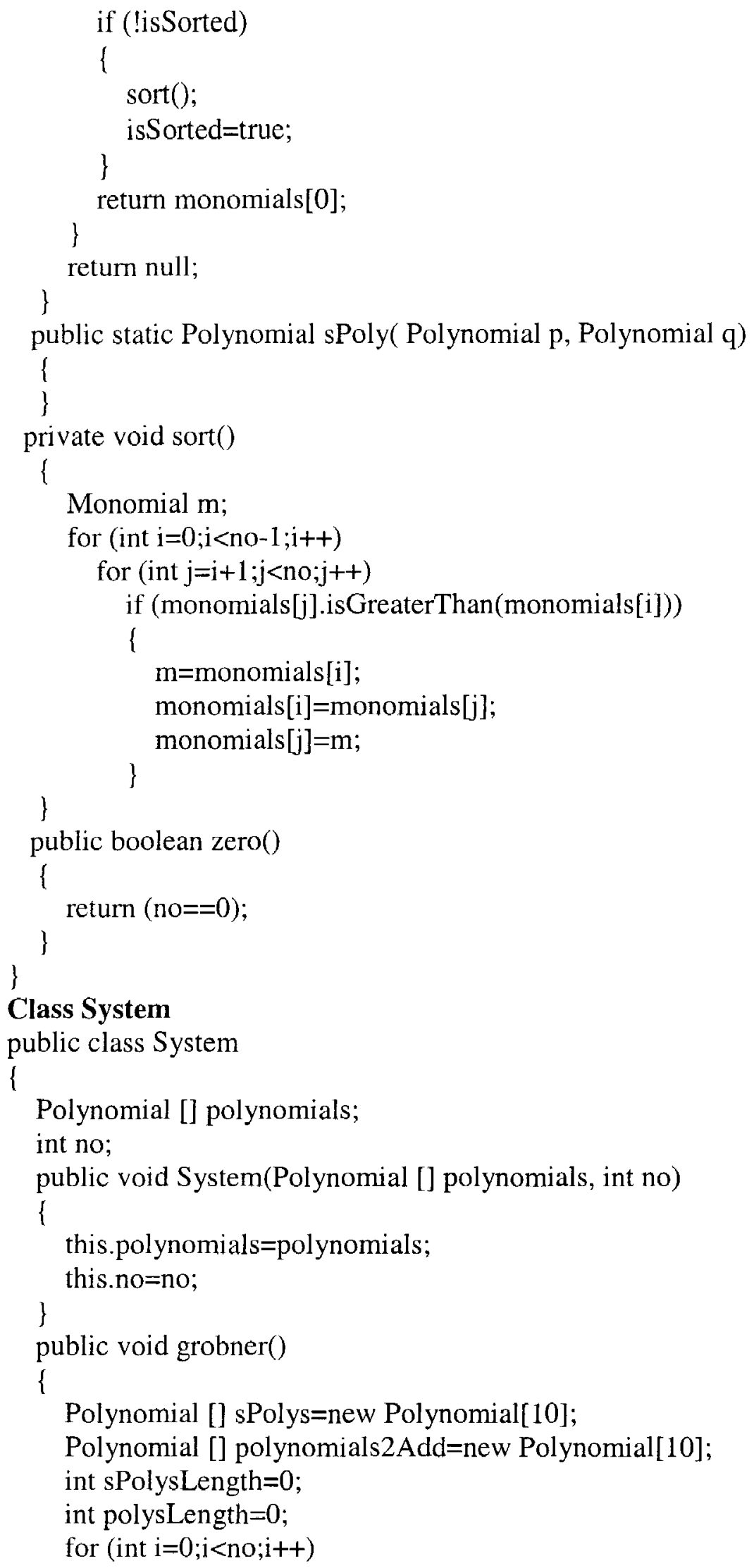




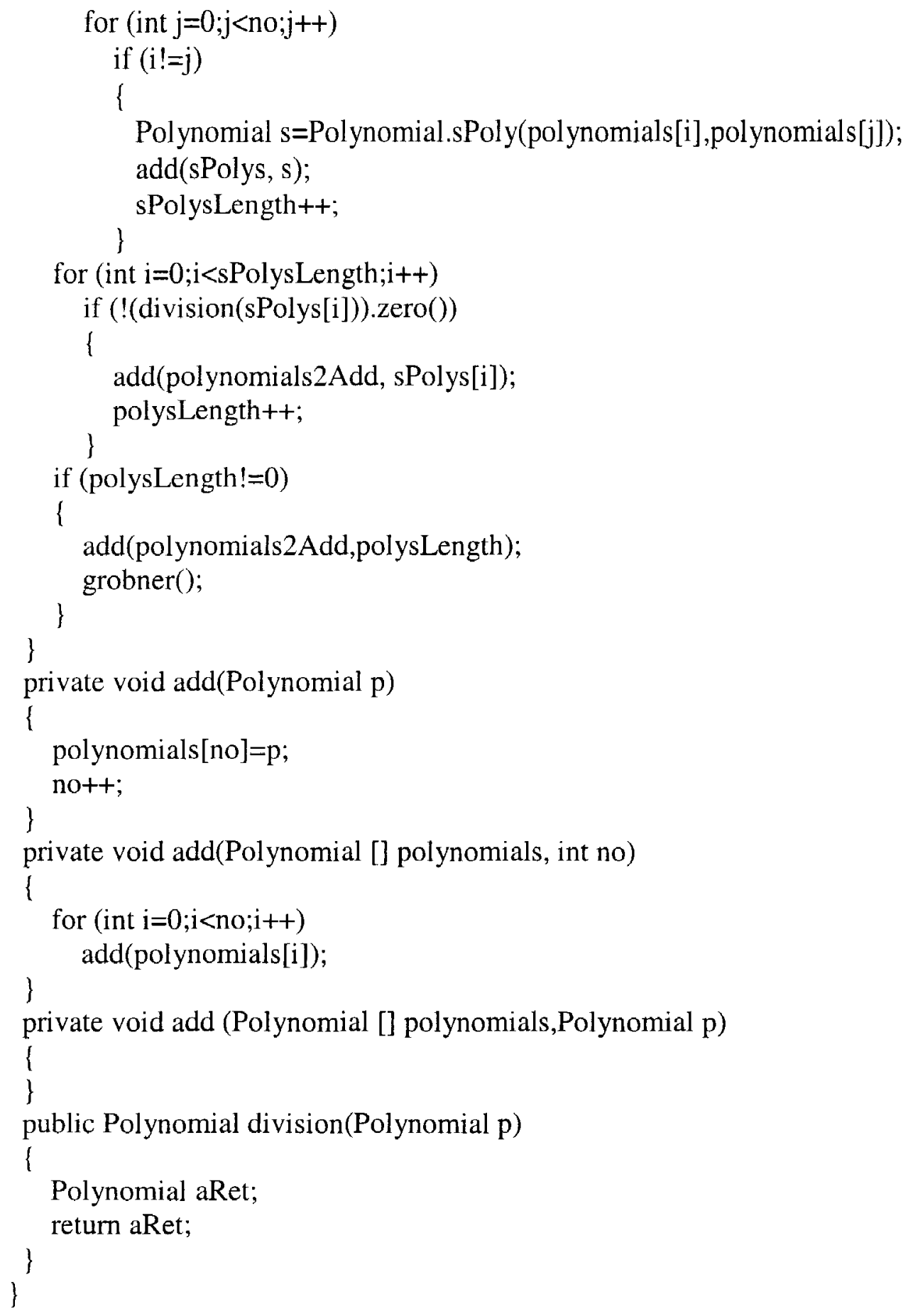




\section{APPENDIX B}

\section{Algebraic Geometry and Computer Algebra concepts}

This appendix covers mathematical definitions for concepts used throughout the dissertation. It starts with defining polynomials, ideals, and varieties, and then defines Grobner bases. Finally, the security spectrum algorithm is stated using the Grobner bases terminology.

\section{Polynomials}

A polynomial $\mathrm{P}(\mathrm{X})$ is defined to be a formal expression of the form:

$P(X)=a_{m} X^{m}+a_{m-1} X^{m-1}+\ldots+a_{1} X^{1}+a_{0}$

Where the coefficients $a_{0} \ldots a_{m}$ are elements of a ring $R$, and $X$ is considered to be a formal symbol, and sometimes called the polynomial variable.

Two polynomials are considered to be equal if and only if the corresponding coefficients for each power of $X$ are equal.

\section{Polynomial Ring R[X]}

The set of all polynomials with coefficients in the ring $R$, together with the addition + and the multiplication mentioned below, forms itself a ring, the polynomial ring over $\mathrm{R}$, which is denoted by $R[X]$.

Polynomial addition, is simply adding up all corresponding coefficients.

Polynomial multiplication is applying the distributive law by multiplying each term in one polynomial by the other and adding up all terms.

\section{Ideal}

Let $R$ be a ring, with $(R,+)$ the underlying additive group of the ring. 
A subset $I$ of $R$ is called right ideal of $R$ if and only if,

1. $(\mathrm{I},+)$ is a subgroup of $(\mathrm{R},+)$,

2. $\mathrm{xr}$ belongs to $\mathrm{I}$ for all $\mathrm{x}$ in $\mathrm{I}$ and all $\mathrm{r}$ in $\mathrm{R}$

A subset I of $R$ is called left ideal of $R$ if and only if,

1. $(\mathrm{I},+)$ is a subgroup of $(\mathrm{R},+)$,

2. $\mathrm{rx}$ belongs to $\mathrm{I}$ for all $\mathrm{x}$ in $\mathrm{I}$ and all $\mathrm{r}$ in $\mathrm{R}$

A two-sided ideal is a left ideal that is also a right ideal, and is often called an ideal.

\section{Ideal generated by a set}

A set $X$ is called the basis of an ideal I if and only if $X$ is a finite subset of I such that any element in I can be expressed as a linear combination of elements in X. The ideal I is also called to be generated from the set $\mathrm{X}$ in this case.

\section{Varieties}

Let $k$ be a closed field and let $\mathrm{A}^{\mathrm{n}}$ be an affine $\mathrm{n}$-space over $\mathrm{k}$. The polynomials $\mathrm{f}$ in the ring $\mathrm{k}\left[\mathrm{x}_{1}, \ldots, \mathrm{x}_{\mathrm{n}}\right]$ can be viewed as $\mathrm{k}$-valued functions on $\mathrm{A}^{\mathrm{n}}$ by evaluating $\mathrm{f}$ at the points in $A^{n}$. For each subset $S$ of $k\left[x_{1}, \ldots, x_{n}\right]$, define the set of zeros of $S$ to be the set of points in $\mathrm{A}^{\mathrm{n}}$ on which the functions in $\mathrm{S}$ vanish:

$Z(S)=\left\{x\right.$ belongs to $A^{n} \mid f(x)=0$ for all $f$ belongs to $\left.S\right\}$.

A subset $V$ of $A^{n}$ is called an affine variety if $V=Z(S)$ for some $S$.

\section{The ideal of a variety}

Given a subset $V$ of $A^{n}$, let $I(V)$ be the ideal of all functions vanishing on $V$, $I(V)=\left\{f\right.$ belongs to $k\left[x_{1}, \ldots, x_{n}\right] \mid f(x)=0$ for all $x$ belongs to $\left.V\right\}$. 
From the above definitions, of the ideal and the varieties, it looks that the ideal and corresponding variety can actually be used to express the same objects using the ideal generated by the system of non linear polynomials representing this object in algebra, and the variety that is the set of all points lying in this intersection area between all those polynomials in geometry.

\section{Grobner Bases}

Grobner basis is a particular kind of generating subset of an ideal $I$ in a polynomial ring $\mathrm{R}[\mathrm{X}]$. It is a finite generating set for an ideal I, characterized by any one of the following equivalent properties, stated relative to some monomial order,

1. The ideal given by the leading terms of polynomials in the ideal $I$ is itself generated by the leading terms of the basis $\mathrm{G}$,

2. The leading term of any polynomial in I is divisible by the leading term of some polynomial in the basis $\mathrm{G}$,

3. Multivariate division of any polynomial in the polynomial ring $\mathrm{R}$ by $\mathrm{G}$ gives a unique remainder,

4. Multivariate division of any polynomial in the ideal $\mathrm{I}$ by $\mathrm{G}$ give 0 .

The third property listed above is the one used as a criterion in the security spectrum algorithm below.

\section{Uniform word problem algorithm,}

Let $f, p_{1}, p_{2}, \ldots p_{n}$ belong to $R[X]$, for some ring $R$, and let $I\left[p_{1}, p_{2}, \ldots p_{n}\right]$ be the ideal generated by the polynomials $\mathrm{p}_{1}, \mathrm{p}_{2}, \ldots \mathrm{p}_{\mathrm{n}}$, The uniform word problem is the decidability problem: 
Does the polynomial $f$ belong to $I\left[p_{1}, p_{2}, \ldots p_{n}\right]$ or not?

To find the solution to that problem, use the extended Euclidean algorithm and divide $f$ by $\mathrm{p}_{1}, \mathrm{p}_{2}, \ldots \mathrm{p}_{\mathrm{n}}$, and check if remainder is zero or not. The problem is that this division does not have a unique solution.

To overcome this challenge, take the ideal $\mathrm{I}\left[\mathrm{p}_{1}, \mathrm{p}_{2}, \ldots \mathrm{p}_{\mathrm{n}}\right]$ and convert its current basis $\left[\mathrm{p}_{1}, \mathrm{p}_{2}, \ldots \mathrm{p}_{\mathrm{n}}\right]$ into a Grobner basis.

Now, the division can take place and property 3 in the definition of Grobner basis can be used to reach a solution.

\section{Security Spectrum algorithm using Grobner bases notations}

1. Gather the intrinsic conditions of each of the two parties.

2. Gather the physical conditions of the communication between the two parties.

3. Model all these conditions to generate a system of (non linear) polynomials, $\left\{p_{1}, p_{2}, \ldots p_{n}\right\}$, describing the whole system of communication.

4. This set of polynomials is to be used to generate a Grobner basis for the ideal $\mathrm{I}\left[\mathrm{p}_{1}, \mathrm{p}_{2}, \ldots \mathrm{p}_{\mathrm{n}}\right]$ in the ring $\mathrm{R}\left[\mathrm{X}_{1}, \mathrm{X}_{2}, \ldots \mathrm{X}_{\mathrm{k}}\right]$ where $\mathrm{R}$ is the set of real numbers and $\mathrm{X}_{1}, \mathrm{X}_{2}, \ldots \mathrm{X}_{\mathrm{k}}$ are the modeling symbols.

5. The security spectrum of the two parties is the Grobner basis generated above.

6. Party A generates a linear combination of the polynomials constructing the spectrum, to be the secret $\mathrm{f}$.

7. Encode the secret, and send to party $\mathbf{B}$.

8. Party B decodes the secret.

9. Party B runs the uniform word problem algorithm; divide the secret polynomial $f$ by the Grobner basis representing the spectrum, and check if remainder of the 
division is zero or not, to decide uniquely if the secret sent is correlated with the spectrum.

10. Party B, runs the correlation level algorithm to determine the level of correlation and decides if this level of correlation authorizes party A. The correlation algorithm is to be run if the remainder of the Euclidean algorithm is not zero. This is basically a statistics function that checks out the degree of the resultant remainder to check out how far it is from the given threshold. 


\section{CURRICULUM VITAE}

Waleed EISaidl

PhD Computer Science and Engineering, WaleedEl_Said@Yahoo.com

\section{PROFESSIONAL, SUMMARY}

Around seventeen years of experience in Computer Science and Information Technology, My experience grew in two dimensions, research in computer science and software engineering. My research interests include network security, cryptography, mathematical modeling, cloud computing, computer algebra, information retrieval, image processing, robotics, and software engineering. I came from strong mathematical background, and I'm trying to use the abstract algebra approaches like the polynomial ring theory to model things like robotics motion, network boundary and intrinsic conditions of communications as well as security constraints, relying conditions around some decision to be used in decision making systems. I'm also using algebraic modeling and in some cases transform non linear polynomial systems into linear ones and extend image processing and computer vision algorithms for edge detection and the like. I'm interested in software engineering and software architecture field and I created a few frameworks and new design patterns at the presentation and business logic tier as well as a framework for an abstract all-purpose generic web service. I used my algebraic modeling ideas to build algorithms for solving robotic kinematics motion problem and for building a prototype solution for the geometric automatic theorem proving in Egypt, and for this I got best MS thesis award for the year 2000. I also used these mathematical modeling ideas to build explosives detection model based on image processing techniques for a homeland security funded project at university of Louisville USA. I also created a couple of security algorithms and frameworks based on novel cryptographic algorithms in my $\mathrm{PhD}$ work in the department of computer engineering and computer science, speed school of engineering, university of Louisville.

\section{PROJEC'TS}

Project: SCADA Network Security November 2006 - November 2009

\section{Role : Research Associate}

Contributions/Achievements:

1. Created a novel cryptographic approach for securing the SCADA network and named it the double secrecy algorithm,

2. Built a java code simulating the communication security using SSL and the double secrecy. The double secrecy found to have a performance time in range of the milli-seconds whereas the SSL performance is in the range of seconds,

3. Extended the double secrecy algorithm to the N-Secrecy,

4. Created the security spectrum algorithm as another novel cryptographic approach extending the security from the using prime numbers and the complexity of integer factorization problem to the polynomial ring theory and algebraic modeling. 
Project : Lxplosives and explosives related compounds detection September 2006 - August 2007

Role : Research Associate / Solutions Architect

Contributions/Achievements:

1. Built an explosives detection framework based on edge detection techniques of the IMS corresponding spectra,

2. Built the whole architecture and design of the system,

Project : Wireless Electronic Monitoring System for Securing Milk from Farm to Processor,

Fel)ruary 2007 - March 2007

Role : Rescarch Associate / Solutions Architect

Contributions/Achievements:

1. Built an end to end architecture for the whole system.

\section{EDUCATION:}

- Certificate: PhD in Computer Science and Engineering, at Speed School of Engineering, University of Louisville, Kentucky, on Network Security, 2010.

- Certificate: MS in Computer Science, Sept,2000, Department of Mathematics, Faculty of Science, Ain Shams University, Cairo, Egypt.

- Certificate: BS of Pure Mathematics and Computer Science May 1993, Faculty of Science, Ain Shams University, Cairo, Egypt.

Grade : Excellent with honor degree.

Position : First Place in 1993 graduation class.

\section{PUBLICATIONS:}

- Security

1. N-Secrecy Authentication Response to Graduated Threat Levels in SCADA Networks: James H. Graham and Waleed H. El-said; S4 SCADA Security Scientific Symposium 2007, Miami, USA, January $24-25$.

2. Double Secrecy: An Enhanced Cryptographic Approach for SCADA System Security: Waleed H. El-said and James H. Graham; International conference on Computer Applications in Industry and Engineering, Nov 4-6, 2009.

- Modeling

3. Explosives Detection, System Modeling and Architecture: Waleed H. ElSaid, and Suraj M. Alexander; Proceedings of the Thirteenth International Society of Science and Applied Technologies (ISSAT), International Conference on Reliability and Quality in Design, Seattle, Washington, USA, August 2-4,2007.

- Architectures and Design Patterns

4. Pipeline To Visitor, a new design pattern for improving maintainability of the SW: Waleed H. El-Said; TheServerSide, June, 2006.

5. Blank Table Template Presentation Framework: Waleed H. El-Said; Proceedings of the Thirteenth International Society of Science and Applied Technologies (ISSAT), International Conference on Reliability and Quality in Design, Seattle, Washington, USA, August 2-4,2007. 
AWARDS:

- Awarded a scholarship to study $\mathrm{PhD}$ in USA, university of Louisville Kentucky.

- Awarded the best MS thesis in Faculty of Science in Ain Shams University Science Day (March, 2002). 